Institute of Pharmaceutical Technology and Regulatory Affairs Faculty of Pharmacy

University of Szeged

Head:

Ildikó Csóka, Pharm.D., Ph.D., dr.habil.

\title{
FORMULATION AND INVESTIGATION OF INNOVATIVE DRUG DELIVERY SYSTEMS FOR THE TREATMENT OF PERIODONTITIS
}

Ph.D. Thesis

by

Attila Léber, Pharm.D.

Supervisors:

Erzsébet Csányi, Pharm.D., Ph.D., dr.habil.

Mária Budai-Szücs, Pharm.D., Ph.D.

Szeged

2020 


\section{PUBLICATIONS RELATED TO THE SUBJECT OF THE THESIS}

I. Attila Léber, Mária Budai-Szűcs, Edit Urbán, Péter Vályi, Anita Kovács, Szilvia Berkó, Erzsébet Csányi: Formulation and Investigation of a Lipid Based Delivery System Containing Antimicrobials for the Treatment of Periodontal Disease. CURRENT DRUG DELIVERY 15(6): 887-897., 2018

IF: 1.645

II. Attila Léber, Mária Budai-Szücs, Edit Urbán, Péter Vályi, Attila Gácsi, Szilvia Berkó, Anita Kovács, Erzsébet Csányi: Combination of Zinc Hyaluronate and Metronidazole in a Lipid-Based Drug Delivery System for the Treatment of Periodontitis. PHARMACEUTICS 11(3): 142., 2019

IF: 4.773

III. Mária Budai-Szücs, Attila Léber, Lu Cui, Muriel Józó, Péter Vályi, Katalin Burián, Balázs Kirschweng, Erzsébet Csányi, Béla Pukánszky: Electrospun PLA Fibers Containing Metronidazole for Periodontal Disease. DRUG DESIGN DEVELOPMENT AND THERAPY 14: 233-242., 2020

IF: 3.208 


\section{ABSTRACTS RELATED TO THE SUBJECT OF THE THESIS}

I. Attila Léber, Mária Budai-Szücs, Edit Urbán, Péter Vályi, Erzsébet Csányi: Development of a lipid-based drug delivery system containing antibiotics for the treatment of periodontitis. $7^{\text {th }}$ BBBB International Conference on Pharmaceutical Sciences, 5-7 October 2017, Balatonfüred, Hungary (PP)

II. Attila Léber, Erzsébet Csányi, Mária Budai-Szücs: Effectiveness of a lipid-based subgingival system for the treatment of periodontitis. $12^{\text {th }}$ Central European Symposium on Pharmaceutical Technology and Regulatory Affairs, 20-22 September 2018, Szeged, Hungary (PP)

III. Léber Attila: Fogágybetegség kezelésére szolgáló lipid alapú hordozórendszer fejlesztése és vizsgálata. XIII. Clauder Ottó Emlékverseny, 22-23 November 2018, Budapest, Hungary (VP)

IV. Attila Léber, Erzsébet Csányi, Mária Budai-Szücs: Lipid-based delivery systems for periodontitis treatment. I. Symposium of Young Researchers on Pharmaceutical Technology, Biotechnology and Regulatory Science, 31 January 2019, Szeged, Hungary (VP)

V. Léber Attila, Budai-Szücs Mária, Vályi Péter, Urbán Edit, Berkó Szilvia, Kovács Anita, Csányi Erzsébet: Antibiotikumot tartalmazó, lipid alapú, helyi hatóanyag-hordozó rendszer vizsgálata. Gyógyszertechnológiai és Ipari Gyógyszerészeti Konferencia, 26-28 September 2019, Siófok, Hungary (PP)

VI. Attila Léber, Erzsébet Csányi, Mária Budai-Szücs: PLA-based nanofibrous systems for the treatment of periodontal disease. II. Symposium of Young Researchers on Pharmaceutical Technology, Biotechnology and Regulatory Science, 23-24 January 2020, Szeged, Hungary (VP) 


\section{TABLE OF CONTENTS}

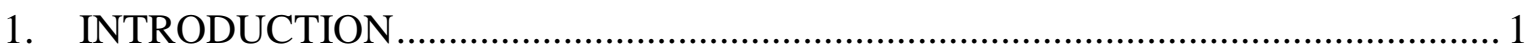

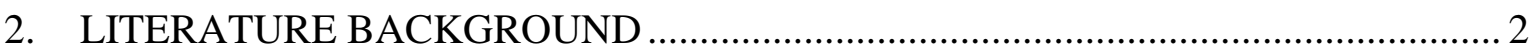

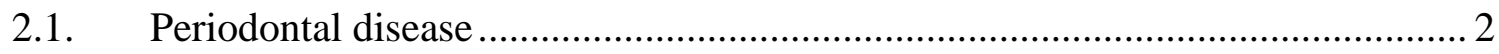

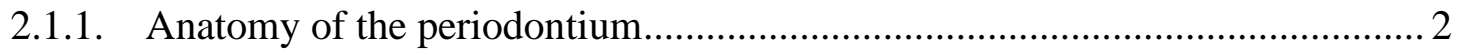

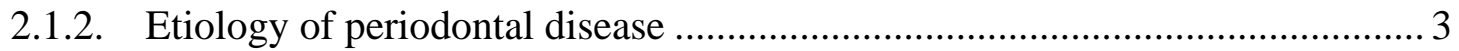

2.2. Treatment methodologies for periodontal disease ......................................... 3

2.2.1. Nonsurgical and surgical periodontal therapies............................................. 4

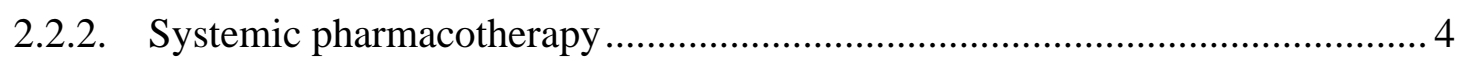

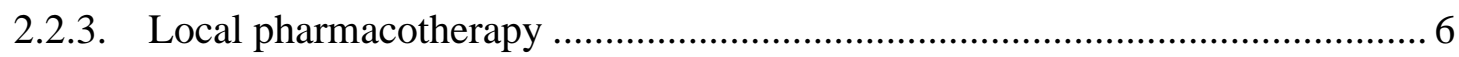

2.2.3.1. Local drug delivery systems in periodontal therapy .............................. 6

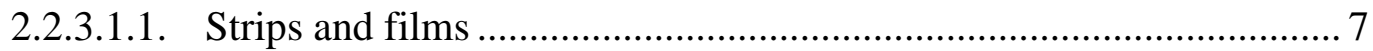

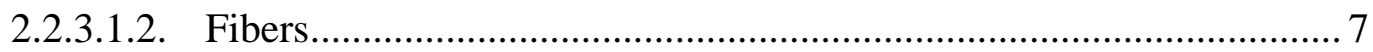

2.2.3.1.3. In situ forming gels as delivery systems ....................................... 8

2.2.3.1.4. Microparticulate systems ............................................................ 9

2.2.3.1.5. Nanoparticulate systems ............................................................. 9

2.2.3.2. Marketed local drug delivery systems .................................................. 9

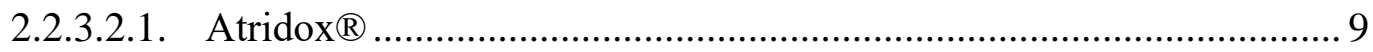

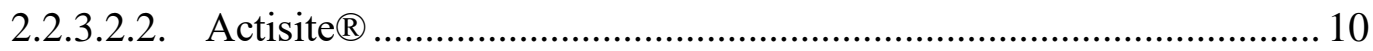

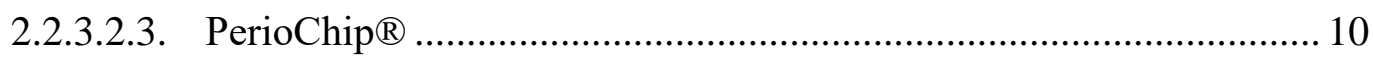

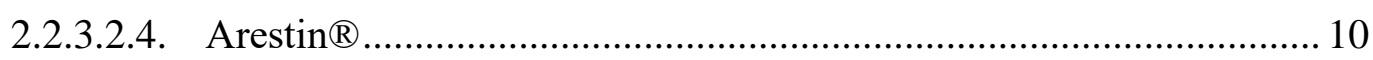

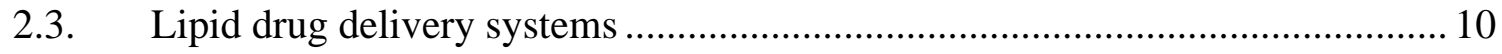

2.3.1. Lipid-based oral drug delivery systems ..................................................... 10

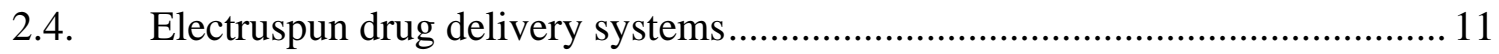

2.4.1. The electrostatic fiber spinning method ..................................................... 12

2.4.2. Applications of electrospun drug delivery systems .................................... 12

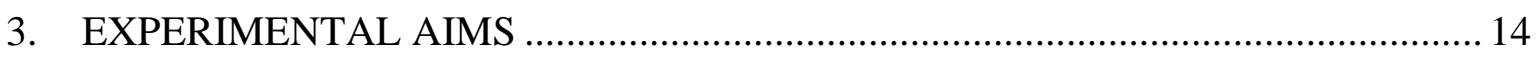

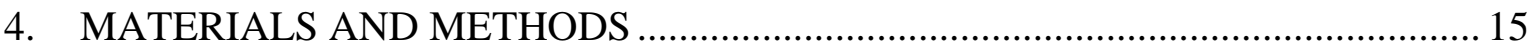

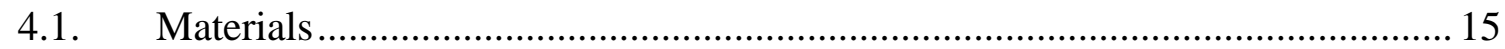

4.1.1. Active agents incorporated into the delivery systems ............................... 15

4.1.2. Materials used for the lipid-based systems ................................................ 15

4.1.3. Materials used for the electrospun nanofibrous systems ............................... 16

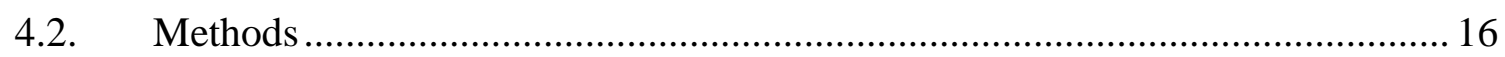




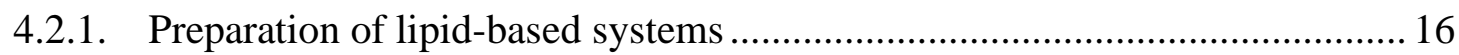

4.2.2. Preparation of PLA-based nanofibrous drug delivery systems ..................... 18

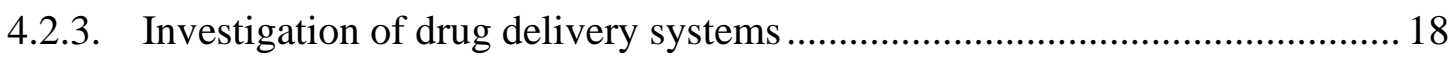

4.2.3.1. Optical contact angle measurements.................................................. 18

4.2.3.2. Differential scanning calorimetry of lipid-based systems ....................... 18

4.2.3.3. X-ray diffraction (XRD) measurements ................................................ 19

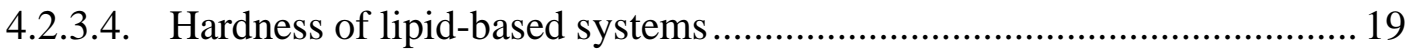

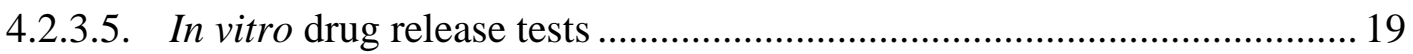

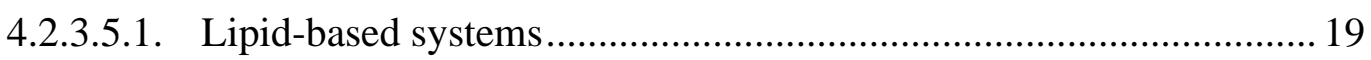

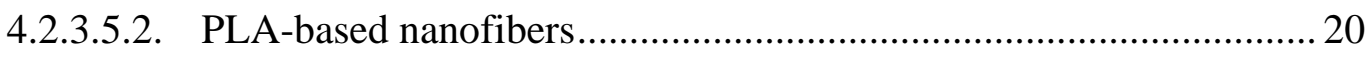

4.2.3.6. Antimicrobial effectiveness of formulations ....................................... 20

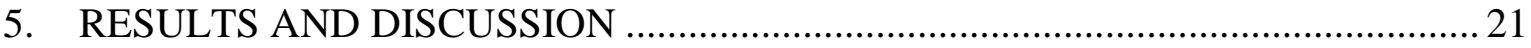

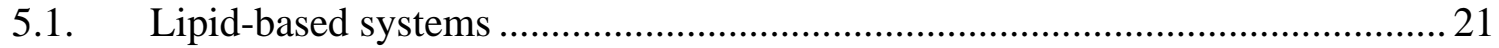

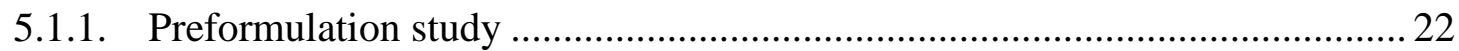

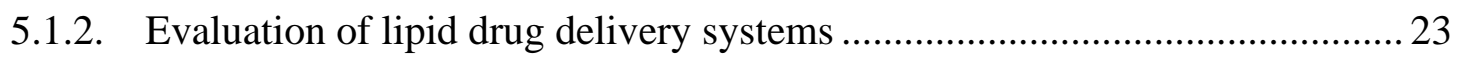

5.1.2.1. Differential scanning calorimetry of formulations ................................ 23

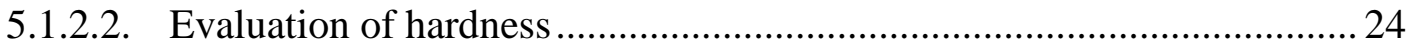

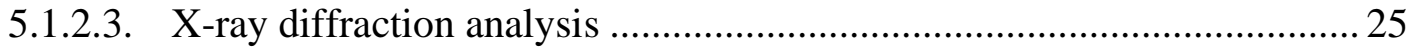

5.1.2.4. Drug diffusion from the delivery systems ......................................... 27

5.1.2.5. Antimicrobial effectiveness of formulations ........................................ 29

5.1.3. Summary of the formulated lipid-based drug delivery systems ..................... 32

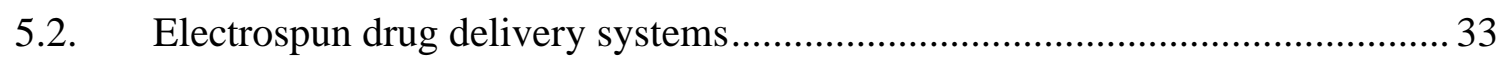

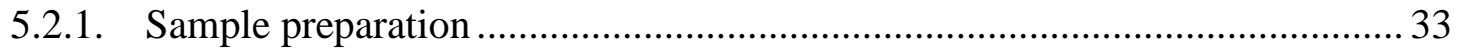

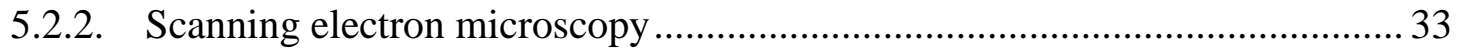

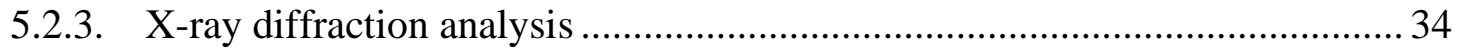

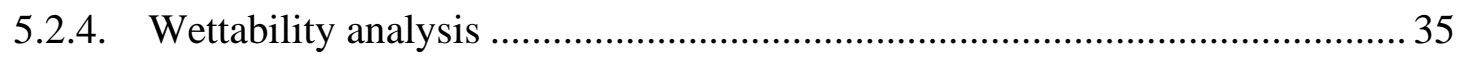

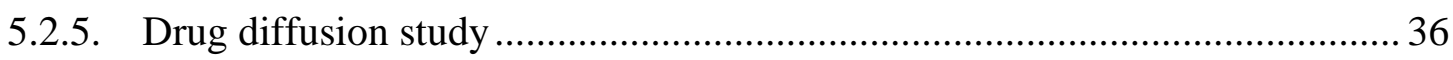

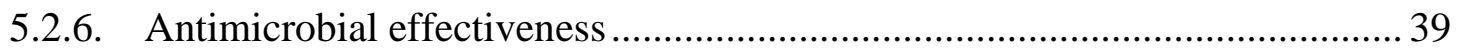

5.2.7. Summary of formulated nanofiber systems ................................................ 41

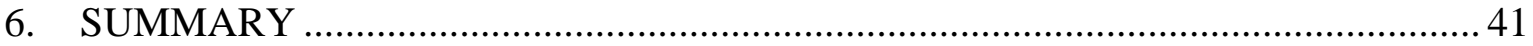

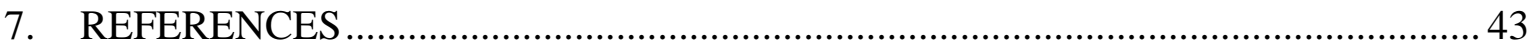




\section{ABBREVIATIONS}

AMX

API

CA

CHX

DSC

HA

HPMC

KP

$\mathrm{MZ}$

NMP

OCA

PD

PDL

PLA

PLGA

PP

SBP

WB

XRD

ZnGlu

ZnHA amoxicillin

active pharmaceutical ingredient

cetostearyl alcohol

chlorhexidine

differential scanning calorimetry

hyaluronic acid

hydroxypropyl methylcellulose

Kolliphor RH40

metronidazole

$\mathrm{N}$-methyl-2-pyrrolidone

optical contact angle (measurements)

periodontitis/periodontal disease

periodontal ligament

polylactic acid

poly(L-lactide-co-glycolide)

periodontal pocket

Suppocire BP

white beeswax

$\mathrm{X}$-ray diffraction

zinc gluconate

zinc hyaluronate 


\section{INTRODUCTION}

The plaque-induced forms of periodontal diseases are the most prevalent chronic inflammatory conditions seen in humans worldwide, affecting nearly half of the adult population. Periodontitis is a major health problem reducing the quality of life and causing tooth loss, disability, masticatory dysfunction, poor nutritional status and compromised speech. Periodontitis is also independently associated with systemic chronic inflammatory diseases including atherogenic cardiovascular disease, type 2 diabetes mellitus, rheumatoid arthritis, chronic kidney disease, obesity and chronic obstructive pulmonary disease [1-3].

Possible therapeutic methodologies include scaling and root planing and surgical intervention, all of which are supplemented by the administration of systemic antibiotics and local chlorhexidine-containing mouthwashes. By scaling and root planing, the debridement of deep pockets is often difficult, while recovery after surgical intervention is long. Using adjuvant systemic antibiotics burdens the patient as adverse events may be observed; and this does not provide a suitable concentration of the active agent in the periodontal pockets. Moreover, the administration of adjuvant antibiotics may contribute to the emergence of resistant bacterial strains [4-9].

In the last 10-15 years, local drug delivery systems received considerable attention. Numerous publications focused on local delivery systems containing antimicrobial drugs. It was established that the application of local drug delivery systems alone or in combination with other dental procedures may result in a more efficient treatment compared to the systemic administration of antimicrobial drugs [4,5,8-11]. Therefore, local delivery systems with incorporated antibiotics is a promising approach to treating periodontitis. Subgingival administration of these systems is most prevalent in aiming at the increased bioavailability of drug formulations. Compared to systemic drug delivery, 100-fold larger concentrations of the antimicrobial agents can be achieved at subgingival sites. Locally administrable formulations may provide prolonged liberation of antimicrobial agents and protection from decomposition in hydrophilic media. These systems may also mask the unpleasant taste of drugs, and may prevent the emergence of antimicrobial resistance, and the appearance of side effects of oral antibiotic use.

Considering the expected properties of formulations, there is a great need for the development of topically used ideal carrier systems, which would allow a wider, safer and more promising application of active ingredients. 


\section{LITERATURE BACKGROUND}

\subsection{Periodontal disease}

Periodontitis is predominantly a bacterial infection involving the accumulation of different bacteria on the non-shedding surfaces of the oral cavity. In susceptible patients, dental plaque comprises periodontal pathogenic microorganisms that initiate and trigger a dysfunctional inflammatory immune response which destroys the supporting tissues. Microbial dysbiosis is a necessary, but alone insufficient condition for the development of periodontal destruction. The host response to the initiating periodontopathogens is predominantly a genetically determined non-modifiable risk factor. Most of the systemic factors are candidates for modification or elimination, which plays a role in changing the susceptibility or resistance of individuals to the treatment. Such modifiable factors are smoking, poorly controlled diabetes mellitus, obesity, stress, osteopenia and inadequate intake of calcium and Vitamin D [1]. The most important modifiable local risk factor is poor oral hygiene, which may initiate the infective inflammatory process in periodontal tissues.

\subsubsection{Anatomy of the periodontium}

The periodontium is a complex structure composed of the gingiva, periodontal ligament (PDL), cementum and alveolar bone. The primary functions of the periodontium are to attach the tooth to the bone and to protect the underlying structures from the oral microflora. The epithelium of the gingiva is composed of stratified squamous epithelium, which is primarily orthokeratinized or parakeratinized [12]. The gingiva covers the supracrestal surface of the root and the alveolar bone and includes epithelial and connective tissue. The transition from gingival to sulcular and junctional epithelium is near the tooth surface. The gingival sulcus is a shallow opening that is bound apically by the coronal aspect of the junctional epithelium, laterally by the sulcular epithelium, and medially by tooth surface, and superiorly exits into the oral cavity. The junctional epithelium is supported by the supracrestal connective tissue fibers of the gingiva. These structures create an initial barrier between the bacterial plaque and the subepithelial components of the periodontium. Apically, the PDL (connective tissue attachment) connects the root cementum to the alveolar bone, functionally supporting the tooth within the mandible or maxilla. The portions of the PDL fibers that are embedded in cementum and alveolar bone are called Sharpey's fibers. In addition to providing tooth support, the unique design of the periodontium allows for the distribution of the forces of mastication [13]. 


\subsubsection{Etiology of periodontal disease}

Inflammation of the gingiva (or gingivitis), is the most common form of gingival disease. This is a result of the presence of local irritants, such as the toxins of the microorganisms related to dental plaque and calculus. The inflammation can result in ulcerative, necrotic and proliferative changes in the gingival tissues. The gingival sulcus can deepen, which may cause harm to the supporting periodontal tissues. Injury of these supporting tissues may become irreversible. Persistence of inflammation can result in hyperplastic changes of the gingival tissues and extension of the crest of the gingival margin toward the crown [14]. The normal protective behavior of the sulcus and the junctional epithelium becomes dysfunctional as inflammation causes a change in the quality of these structures and tissue proliferation. A pocket is formed from the sulcus; therefore, microorganisms and their toxins can access the exposed subgingival tissues which undergo further pathologic changes. The persistence of this process may result in the separation of the epithelial junction from the root and further enlargement of the pocket. The extension of inflammation from the margin of the gingiva into the supporting periodontal tissues marks the transition from gingivitis to periodontitis. The essential problem of periodontal disease is the destruction of the alveolar bone with a loss of crestal height and PDL destruction. Untreated periodontal disease may lead to the loosening of the teeth and eventually tooth loss [12,13].

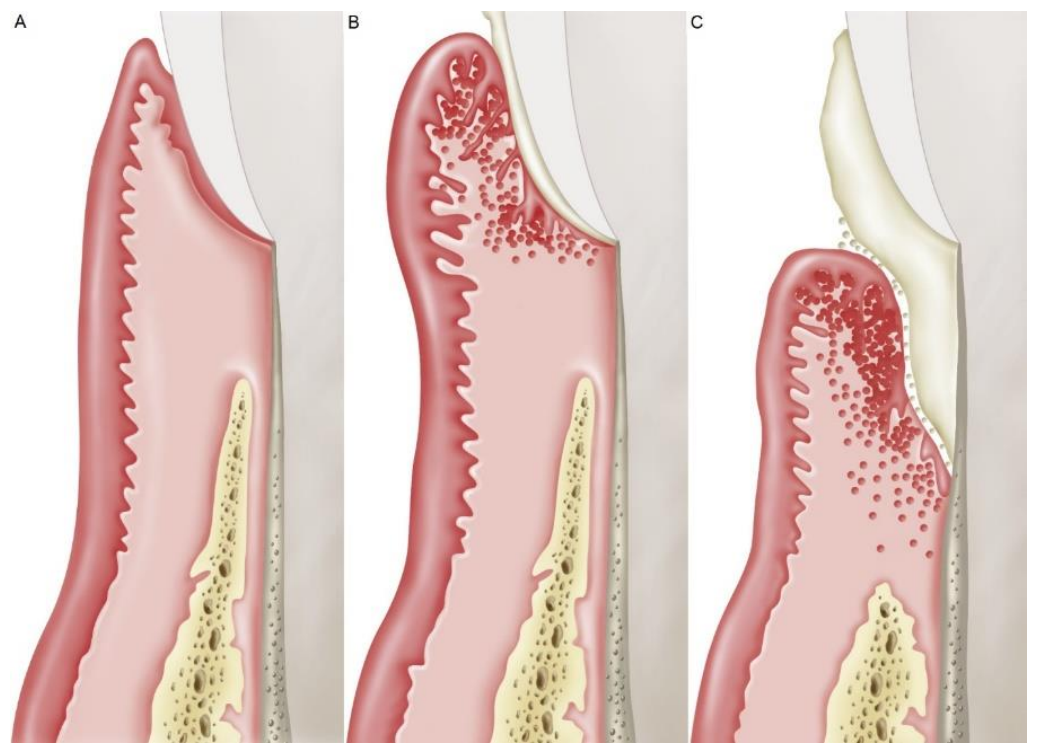

Figure 1. Progression of periodontal disease. A: healthy gingiva; B: gingivitis; C: periodontitis [12].

\subsection{Treatment methodologies for periodontal disease}

Treatment of periodontitis involves a fine balance of various non-surgical and surgical methods carried out in order to reduce periodontal pocket depth, access residual plaque, initiate the regeneration of periodontal supporting tissues and decrease the risk of disease progression. 
Subgingival anti-infective therapy performed together with self-performed plaque control provides significant benefits in clinical parameters and improvement of systemic inflammatory markers [15].

\subsubsection{Nonsurgical and surgical periodontal therapies}

Non-surgical periodontal treatment consists of professional removal of plaque and calculus, elimination of plaque retentive factors, oral hygiene instruction, chemical plaque control and antibiotic medication. Treatment of teeth with tooth/site-dependent factors is less predictable and bears an elevated risk of the progression of periodontal tissue breakdown [16]. Mechanical therapy alone may have limited effect on some periodontopathogens and fail to eliminate them in ecological niches [15].
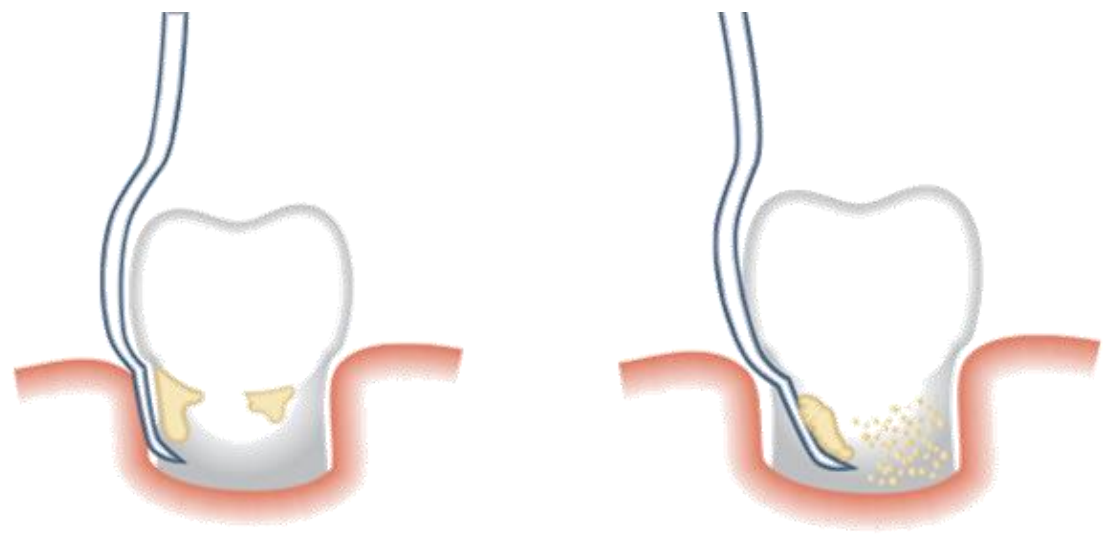

Figure 2. Mechanical debridement of subgingival tooth surfaces [17].

When pocket depth reaches $5 \mathrm{~mm}$, mechanical debridement may not provide sufficient removal of subgingival plaque; therefore, surgical therapy may be required. The primary purpose of surgical periodontal therapies is to access residual plaque of deep pockets and to establish a gingival morphology conducible to efficient plaque control. The outcome of periodontal surgery depends on many factors. However, clinical trials show evidence that unless patients maintain rigorous postoperative plaque control, the deepening of periodontal pockets continues. Moreover, costs of these treatment methods are higher and longer recovery periods are needed $[15,18,19]$.

\subsubsection{Systemic pharmacotherapy}

Systemic antimicrobials are applied adjunctively to mechanical non-surgical and surgical treatment and may provide additional benefits in case of very deep pockets or specific microbial infections. Systemic antimicrobial therapy is the most effective when the mechanical disruption of the subgingival biofilm is performed during subgingival debridement $[15,20]$. 
Literature data suggest that a high number of different antimicrobial agents may be effective in the adjunctive treatment of periodontal disease, but selection of the proper one may be dependent on different factors such as patient age, drug allergy, pregnancy, local factors, drug factors and organism related considerations [21]. That is why there is a pool of antibiotics from which the dental expert can choose after individual features have been considered. In Table 1, a summary of these active agents, their average doses and duration of treatment are shown.

Table 1. Antibiotics, their dose, frequency of dosage and duration of therapy in the systemic antimicrobial therapy of periodontal disease.

\begin{tabular}{lccc}
\hline & Dose & Frequency & Duration \\
\hline Amoxicillin & $250-1000 \mathrm{mg}$ & $\begin{array}{c}\text { twice, three times } \\
\text { daily }\end{array}$ & $7-14$ days \\
\hline Azithromycin & $500 \mathrm{mg}$ & once daily & 3 days \\
\hline Ciprofloxacin & $500 \mathrm{mg}$ & twice daily & - \\
\hline Clarithromycin & $500 \mathrm{mg}$ & twice daily & 3 days \\
\hline Doxycycline & $100 \mathrm{mg}$ & once daily & $9-14$ days \\
\hline Metronidazole & $200-500 \mathrm{mg}$ & twice, three times \\
daily & $7-14$ days \\
\hline Tetracycline & $250 \mathrm{mg}$ & four times daily & 21 days \\
\hline
\end{tabular}

Administration of antibiotics may be advantageous if they supplement scaling and root planing therapies, but overall analysis of the clinical trials conducted indicate that a certain combination of two aforementioned agents may provide higher effectiveness [22]. The protocol for the combination of AMX and MZ was proposed by Winkelhoff and colleagues. Their results showed promising effectiveness when this combination was used [23].

In conjunction with the administration of systemic antibiotics, adverse events and antibiotic resistance must be taken into consideration. Most common side effects include headache, malaise, gastrointestinal discomfort, nausea, vomiting and diarrhea. In addition, other disorders affecting the respiratory tract, the skin or the musculoskeletal system may arise. However, these side effects, similarly to the ones listed before, were not reported in the case of local drug delivery systems containing antimicrobial agents due to the local administration and lower concentration of these APIs referring to the entire body [22,24].

Antibacterial resistance is an emerging issue which is due to the careless use of antibiotics [25]. Not following the therapeutic protocol during systemic antibiotic administration may eventuate the emergence of resistant bacteria which can cause treatment failure and tooth loss $[21,24]$. 


\subsubsection{Local pharmacotherapy}

The local delivery of antimicrobial drugs may reduce the systemic adverse effects and provide effective drug concentrations in the periodontal pockets to eliminate the pathogens from the subgingival area [5,26]. Local application of antibiotics has been advocated for patients with localized lesions or a limited amount of non-responding or recurrent sites [15]. In the literature, many articles are about the development of various types of delivery systems (fibers, films, gels, strips, injectable systems, microparticles) containing different active agents (tetracycline, doxycycline, chlorhexidine, clindamycin, metronidazole, amoxicillin, povidoneiodine) $[5,8,34-38,9,27-33]$. The possible classification of local drug delivery systems is shown in Figure 3.

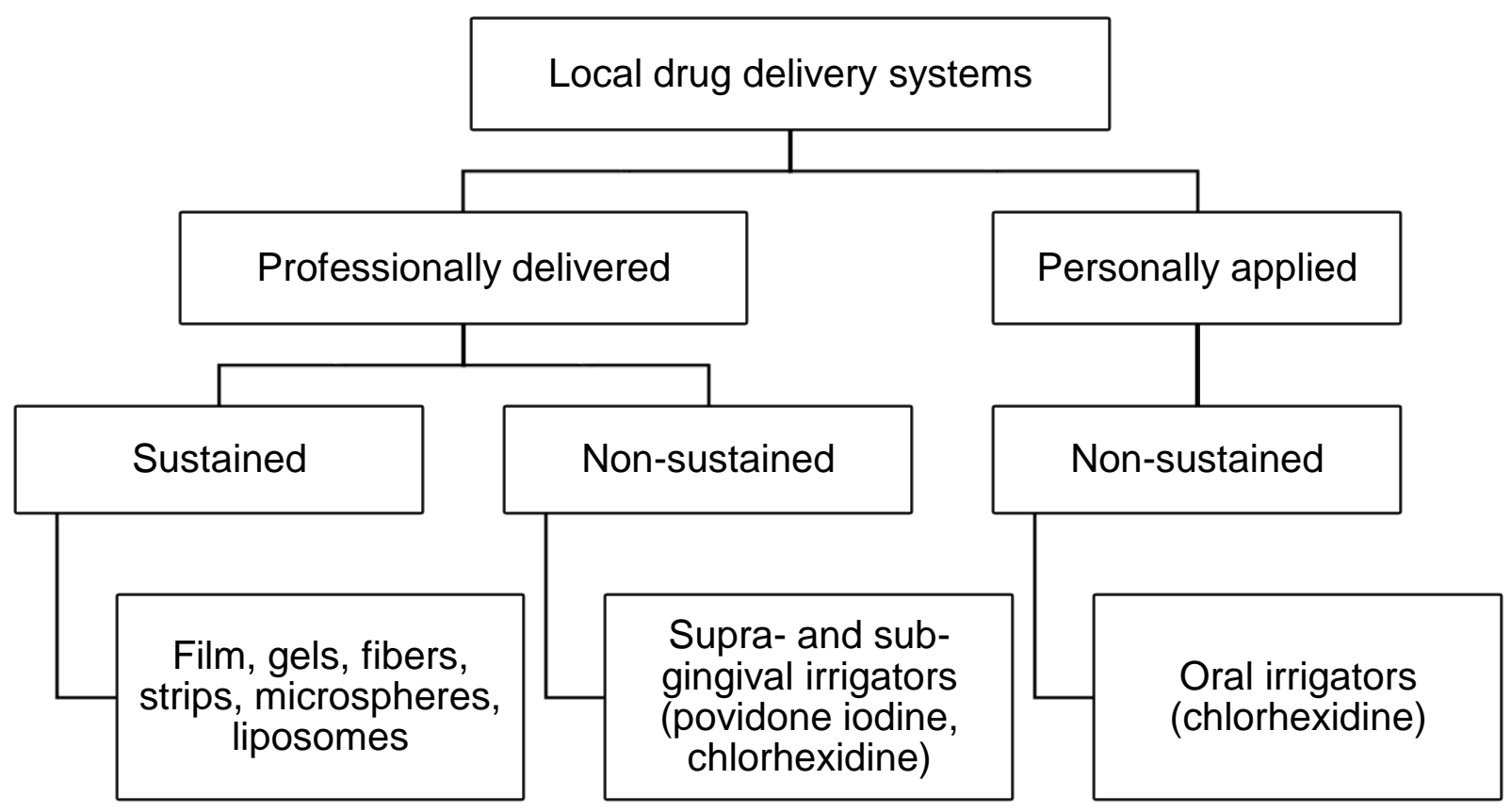

Figure 3. Possible classification of local drug delivery systems used in periodontitis treatment [39].

\subsubsection{Local drug delivery systems in periodontal therapy}

Advantages of local drug delivery systems over systemic antimicrobials are the following: non-invasive and direct administration to the periodontal pockets (site of infection), no first pass metabolism, reduced dose and frequency of drug administration and better patient compliance. Furthermore, agents unsuitable for systemic therapy can be incorporated into them [40]. The features of the ideal local drug delivery system include easy administration, controlled drug release, constant drug concentration for a prolonged period in the periodontal pockets, biodegradability and biocompatibility [41]. 


\subsection{Strips and films}

Strips and films are polymer-based matrix systems. They are designed to provide controlled and sustained delivery of the active agents when placed in the periodontal pocket [4]. Different polymers can be used to create these systems. These polymers can be either biodegradable or non-biodegradable $[42,43]$. Ideal thickness of strips and films is between 5 and $200 \mu \mathrm{m}$.

The drug release from strips and films depends on the biodegradability of the polymer. If the polymer is biodegradable, the drug is released either by diffusion and/or matrix dissolution or erosion. In the case of non-biodegradable polymer matrices, drug release is driven only by matrix diffusion [43].

One of the main advantage of strips and films is easily manipulation of shape and size to match pocket dimensions. This feature allows easy insertion and lack of discomfort [44].

Several studies in literature have used the combination of these polymers to deliver antimicrobials such as doxycycline, tetracycline, metronidazole and chlorhexidine. These systems were proven to be effective in reducing the clinical variables of periodontal disease [41].

\subsection{Fibers}

Polymers used for creating fibers are either natural (gelatin, chitosan and zein) or synthetic (poly(lactide-co-glycolic acid) and polycaprolactone), both can be biodegradable or nonbiodegradable [41].

Drugs are loaded into the fibers by passive and active methods, and the efficacy of the drug loading depends on the properties of the active agent and polymer, and on the formulation method $[45,46]$.

After the introduction of fibers into the periodontal pocket, drug release is primarily driven by diffusion, swelling and degradation. All of these are dependent on the polymer type and its properties like hydrophobicity, hydrophilicity, molecular weight, crystallinity, melting point, etc. Drug release of delivery systems also influenced by the features of the incorporated drug $[47,48]$.

Fibers are highly researched in the field of periodontics, and some of them provided improvement for the patients; therefore, they may be advantageous in the treatment of periodontal disease [39]. 


\subsection{In situ forming gels as delivery systems}

In situ forming delivery systems offer an interesting potential for the treatment of periodontitis. $[49,50]$. Special needles and syringes are used to introduce these systems into the periodontal pocket as liquids. After administration, the injected liquid solidifies or become cross-linked and provides sustained or controlled release of the incorporated API. Advantages include easy administration, adapted form to the geometry and size of the periodontal pocket and easy access to areas that are difficult to reach [51].

Gels are often used in the treatment of different oral diseases such as oral ulcers, denture stomatitis and desquamative gingival lesions. These applications are possible due to features like easy formulation, controlled release, minimum dose frequency and low drug toxicity $[52,53]$.

Different stimuli ( $p \mathrm{H}$, temperature, ions or magnetic force) can induce the cross-linking of gels in the periodontal pocket or special mixing needles can be used during the administration. Mixing needles are special two-compartment devices in which one compartment contains the polymer solution, while the other contains the cross-linking agent. During injection, a special needle is used to mix the content of the two compartments before the mixture is delivered into the periodontal pocket [39].

Various polymers (such as Carbopol, xanthan, carboxy methyl cellulose and chitosan $[43,54])$ can be used to formulate different gels for drug delivery purposes. They can be loaded with a wide range of active agents such as antimicrobials [55], bisphosphonates (alendronates [56], zoledronates [57]), anti-inflammatory drugs and statins [58]. Literature data indicates that local delivery of drug-containing gels has a potential in improving the clinical parameters associated with periodontitis.

In situ forming lyotropic liquid crystalline systems have been also described in the literature for periodontal disease treatment [59]. Lyotropic liquid crystalline systems are formed from water, surfactants, co-surfactants and oils. There is a definite concentration ratio and temperature range in which these systems are formed. An injectable mixture of oils and surfactants can be prepared, which will become a lyotropic liquid crystalline system in the periodontal pockets, when the mixture comes into contact with water or aqueous media. Increased viscosity of systems may lead to longer subgingival retention and sustained release of active agent $[59,60]$. 


\subsection{Microparticulate systems}

Microparticles are spherical polymeric structures in the diameter range of $1-1000 \mu \mathrm{m}$. Different biodegradable and non-biodegradable polymers can be used to create microspheres. Water-soluble (e.g., gelatin, starch) and water-insoluble polymers (such as ethyl cellulose, polyethylene) are also used for microencapsulation [61-64]. The polymeric matrix protects the active agent from incompatibilities and the effects of the environment, masks the unpleasant taste of drugs, enhances bioavailability and provides controlled release [61]. These particles can be incorporated into different systems like gels, pastes or chips to deliver to the periodontal pocket [64].

Results of clinical studies indicate that they may provide adequate concentrations of antimicrobial drugs in the periodontal pockets and contribute to the treatment of the disease [65-69].

\subsection{Nanoparticulate systems}

Nanoparticles (dimension less $\leq 100 \mathrm{~nm}$ ) have gained considerable attention due to their ability to precisely deliver drugs to a target site [70,71]. Drug-loaded nanoparticles are delivered to the site of action directly and can provide sustained and controlled release of APIs. Bone regenerative and antibiotic activity of these delivery systems are important in the treatment of periodontal disease; thus, metallic nanoparticles (copper, silver and titanium dioxide) are under thorough investigation [72-76].

Nanoparticulate systems also include liposomes, polymeric nanoparticles, polymeric micelles and solid lipid nanoparticles [72]. Advantages of these systems include better transport through cell membranes, improved drug loading capacity and biocompatibility [77].

\subsubsection{Marketed local drug delivery systems}

\subsection{Atridox ${ }^{\circledR}$}

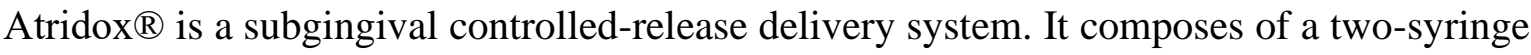
mixing system. One of the syringes contains $450 \mathrm{mg}$ of the Atrigel ${ }^{\circledR}$ Delivery System, which is a bioabsorbable polymeric formulation composed of $36.7 \%$ polylactic acid dissolved in 63.3\% N-methyl-2-pyrrolidone (NMP). The other syringe contains $50 \mathrm{mg}$ of doxycycline hyclate (hydrochloride hemiethanolate hemihydrate) which is equivalent to $42.5 \mathrm{mg}$ doxycycline. After mixing, the product is a yellow viscous liquid which contains $10 \%$ of doxycycline hyclate. When the mixture comes into contact with the cervicular fluid, the liquid 
product solidifies. The solidified mixture allows the controlled release of doxycycline hyclate for 7 days [78].

\subsection{Actisite ${ }^{\circledR}$}

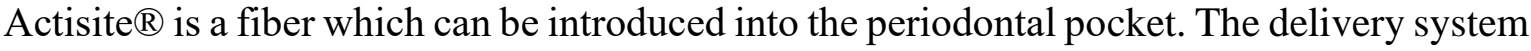
consists of a $23 \mathrm{~cm}$ monofilament of ethylene-vinyl acetate copolymer. This device has a diameter of $0.5 \mathrm{~mm}$ and $12.7 \mathrm{mg}$ of tetracycline hydrochloride dispersed in it. The fiber provides continuous release of tetracycline for 10 days. Unfortunately, the marketing of the device has been discontinued $[79,80]$.

\subsection{PerioChip ${ }^{\circledR}$}

PerioChip ${ }^{\circledR}$ (chlorhexidine gluconate) is a small, orange-brown, rectangular chip which at one end is rounded. This device can be inserted into periodontal pockets. Each PerioChip ${ }^{\circledR}$ has a weight of approximately $6.9 \mathrm{mg}$ and contains $2.5 \mathrm{mg}$ of chlorhexidine gluconate. The biodegradable matrix, in which the chlorhexidine is dispersed, consists of hydrolyzed gelatin which is cross-linked with glutaraldehyde [81].

\subsection{Arestin ${ }^{\circledR}$}

Arestin ${ }^{\circledR}$ is a drug delivery system consisting of microspheres. It is a sustained release product into which $1 \mathrm{mg}$ of minocycline hydrochloride has been incorporated. The matrix of the delivery system is the bioresorbable polymer PLGA [82].

\subsection{Lipid drug delivery systems}

In the last 10-15 years, lipid drug delivery received considerable attention in the field of pharmaceutical technology. Administration of lipid materials is most prevalent in solubility enhancing, lymphatic transport targeting, and/or intestinal transport modulation. Moreover, creating sustained release systems, covering the bitter or bad taste of active ingredients or protecting drug molecules susceptible to different environmental factors may also be a purpose for the application of lipid excipients [83].

\subsubsection{Lipid-based oral drug delivery systems}

In recent years, several oral, lipid-based systems were successfully marketed. That is why there is a considerable interest in these delivery systems. Oral lipid delivery systems can be classified into different subgroups: Type I, Type II, Type IIIA, Type IIIB and Type IV. The 
content of hydrophilic component of formulations is the lowest in Type I and highest in Type IV systems $[84,85]$.

Delivery systems in the first category (Type I) are the simplest lipid products, which means that the active ingredient is dissolved in digestible oil. Triglycerides are easily digested to smaller components in the alimentary tract and a colloidal dispersion is formed in the intestines from where the passive transport of drug containing micelles can occur $[84,85]$.

Formulations in the second category (Type II) contain a lipophilic surfactant which promotes emulsification in the gastrointestinal tract. If the system is carefully composed and under optimum conditions, a self-emulsifying drug delivery system can be formulated. This means that emulsification of the lipid components occurs spontaneously under very gentle conditions. The size of the produced particles is $100-250 \mathrm{~nm}$, but it is heavily influenced by the concentration and type of the surfactant $[84,85]$.

Systems in the third group (Type III) contain hydrophilic surfactants with HLB values over 12, and other water-soluble components. The difference between Type II and III formulations that the hydrophilic components will be dissolved in the aqueous phase after dispersion. They can be self-emulsifying or self-microemulsifying drug delivery systems. Type III formulations can be further divided into two subgroups Type IIIA and Type IIIB. The difference between formulations in group A and group B is that delivery systems in group B have higher hydrophilicity, than in group A $[84,85]$.

Delivery systems in the fourth group (Type IV) contain large amounts of surfactants (or a mixture of surfactants) and co-solvents. There is a high chance that - from these systems - the drug will precipitate and form amorphous or very fine crystals in the gastrointestinal tract. Concerns has been also emerged in connection with these formulations. Firstly, the dissolution of surfactants may take a long time because a gel or liquid crystal phase is formed at the interface. Secondly, high concentration of surfactants can irritate the mucosa of the stomach or intestine and cause damage [85].

\subsection{Electrospun drug delivery systems}

The idea of using high voltage to induce formation of liquid drops has been proposed more than 100 years ago. The electrospinning process was first patented in 1934, but the procedure did not gain commercial success. In the 1990s, interest in this technique was revived and many articles have been published in this topic [86-92].

Electrospinning is a simple method for generating nano or microscale polymer fibers. This technique has received considerable attention in both academic and industrial circles, on the 
account of its unique ability to produce ultrafine fibers of different materials in various assemblies.

\subsubsection{The electrostatic fiber spinning method}

Electrospinning is a method during which a polymeric solution is formed into nano- or micro-sized fibers. In more details, a polymer solution in a syringe is dosed by a pump through a needle onto which high voltage is applied. The solution flowing through the needle with constant flux forms a drop at the end of the needle. Because of the applied high voltage and the grounded metal collector, an electric field is generated. This electric field will force the drop of polymer solution at the end of the needle to form a cone (Taylor cone). When the electric field becomes strong enough to overcome the surface tension, a jet of polymer solution ejected from the cone. On its way to the collector plate, the solvent evaporates from the polymer solution, and solid fibers are formed [93].

Different electrospinning methods exists which were developed from the basic one described above. The second generations of electrospinning techniques are side-by-side electrospinning and co-electrospinning [94].

The third-generation electrospinning technique, multifluidic electrospinning has been proposed and the produced electrospun fibers possess multichannel structures or multiple coreshell structures [94].

As the yield of fibers are low due to the limited flow rate and only one needle, a multi-needle electrospinning technique has been proposed. This technology may improve the yield of fibers which is one of the main research goals of technology [95].

The production rate could be largely improved by the elimination of the needle. During needle-less electrospinning nanofibers are created from a liquid with an open surface. Selforganization of the waves of an electrically conductive liquid occurs when electric voltage reaches a critical level, and multiple jets are generated [96].

\subsubsection{Applications of electrospun drug delivery systems}

Electrospun nanoscale fiber may be used for various applications such as air filtration [97], oil and water separation [98], water purification, disinfection [99] and even sensory functions [100].

However, the most important use of electrospun nanofibers is in the medical field as drug delivery systems or for tissue engineering purposes. These two areas mostly apply nanofibers which were created from biopolymers. Electrospun fibers have been used in vascular, bone, 
neural and ligament tissue applications [101]. For drug delivery purposes, different kind of drugs and biopolymers were investigated to provide sustained release. As the fibers have high surface-to-volume ratio, they can provide suitable media for cell binding and cell proliferation, and also can increase the drug loading capacity [102]. It is also possible to incorporate proteins, DNA, RNA and growth factors into the fibers, while their functionality and bioactivity is maintained [103].

Electrospun drug delivery systems containing antibiotics may be advantageous in the treatment of periodontal disease. In contrast with oral antibiotic treatment, nanofibers could provide high concentrations of antimicrobial drugs at the site of infection for an extended time period.

In a study, tinidazole-containing, biodegradable, chitosan/poly( $\varepsilon$-caprolactone $)$ nanofibrous systems were created. Tinidazole was released in a sustained manner, while an 18-day bacterial growth inhibition could be achieved. The conducted clinical study indicated significant decrease in clinical markers of periodontal disease [90].

In another study, tetracycline hydrochloride was incorporated into PLGA/gum tragacanth nanofibers for periodontal regeneration. Fibers were fabricated via blend and coaxial electrospinning. Drug release was prolonged for an outstanding 75 days. Antimicrobial effectiveness of these delivery systems were also proven [89].

Schkarpetkin and colleagues [91] aimed to prepare nanofibers which contain the combination of ampicillin and $\mathrm{MZ}$ in a PLA matrix. Fibers were spun via multijet electrospinning. Drug release from fibers was sustained for 96 hours and fibers showed antimicrobial effectiveness against Aggregatibacter actinomycetemcomitans, Porphyromonas gingivalis, Fusobacterium nucleatum and Enterococcus faecalis [91]. 


\section{EXPERIMENTAL AIMS}

The aim of my Ph.D. work was to develop and investigate innovative drug delivery systems containing antibiotics for the local treatment or adjuvant local therapy of periodontal disease. Two main research approaches were carried out during my work, the first is the development of lipid formulations, the second is the formulation and investigation of electrospun drug delivery systems for local therapy.

In the case of lipid-based system, components were selected, and the composition was optimized in order to prepare a delivery system which meets the following predefined requirements:

- To contain biocompatible and biodegradable materials.

- To soften at body temperature which allows the delivery system to accommodate to the shape of the periodontal pocket and to leave the periodontal pocket as the gingival tissue heals.

- To have a mucoadhesive feature which helps to maintain the delivery system in the subgingival area and, thus, prolong the effect of the active agent.

- To provide a non-aqueous system in which the incorporated active agent is protected against the effects of the environment (oxidization and humidity) and, thus, decomposition.

- To provide sustained release of the API which contribute to a longer antimicrobial effect.

- To be able to incorporate a wide spectrum of antibiotics or disinfectants to suit the individual needs of patients.

The second research line was focused on the development and characterization of nanofibrous electrospun drug delivery systems. The following requirements were set:

- To have a polymer base which is biocompatible and biodegradable

- To use an adequate polymeric base which provides sustained release of hydrophilic antimicrobial agents.

An important element of the work was the investigation of the relationship between the incorporated API and the bases of the drug delivery systems, and analysis of the antimicrobial effectiveness of the formulations. 


\section{MATERIALS AND METHODS}

\subsection{Materials}

\subsubsection{Active agents incorporated into the delivery systems}

Amoxicillin (AMX) is a semi-synthetic penicillin derivative, is known to be effective against many aerobic or anaerobic Gram-positive and Gram-negative bacteria [4,104]. Literature data shows that AMX is widely used in the treatment of periodontal disease.

Metronidazole (MZ) is an antibacterial agent, included in the nitroimidazole group. Numerous bacteria and protozoa — even anaerobes — are known to be susceptible to this API $[5,105] . \mathrm{MZ}$ is also used in the treatment of periodontitis.

Chlorhexidine gluconate ( $\mathrm{CHX})$ is a biguanide antiseptic widely used in the treatment of periodontal diseases as mouthwashes. This agent is active against Gram-positive and Gramnegative organisms, facultative anaerobes, aerobes, and yeasts, and also reduces the build-up of plaque and may improve the condition of patients in mild gingivitis.

Zinc hyaluronate ( $\mathrm{ZnHA})$ is a polymer derived from sodium hyaluronate and regularly applied in topical gels to accelerate the healing of wounds of different etiologies and to decrease the chance of a possible bacterial superinfection [106]. It may be used as a mucoadhesive agent in ophthalmic solutions to lengthen clearance and provide higher efficiency [107]. Moreover, hyaluronic acid shows anti-inflammatory effects [108], which makes it suitable for the treatment of periodontal diseases. That is why this component was also used in the formulations as a gelling and mucoadhesive agent and to provide an antimicrobial effect.

Zinc gluconate (ZnGlu) is a zinc salt with a known anti-microbial effect. It is often used in combination with zinc hyaluronate in order to increase the antimicrobial effect.

\subsubsection{Materials used for the lipid-based systems}

Cetostearyl alcohol (CA) was used as a potential structure-building component. It is mostly used as a surfactant, a co-surfactant or as a viscosity increasing agent in semisolid formulations. The melting range of the pure component is between $48-56^{\circ} \mathrm{C}$ [109].

White beeswax (WB) was also used as a potential structure-building component. It is a chemically bleached natural beeswax which consists of esters of C24 to C36 straight-chain alcohols and straight-chain acids. It is used in creams, oils, ointments to increase consistency and in emulsions as a stabilizer. According to the literature, its melting point is between 60 $67^{\circ} \mathrm{C}[109]$. 
Lipophilic Suppocire BP (SBP) was chosen as a lipid base. It has a melting range between $36-37{ }^{\circ} \mathrm{C}$ and a hydroxyl value between 30 and 50 . In most cases, it is used as a base for suppositories [109].

Methocel E4M (hydroxypropyl methylcellulose, HPMC) was used as a gelling and mucoadhesive component. HPMC is often used as a mucoadhesive agent in oral formulations such as films [110] or buccal tablets [111] to increase the residence time of the delivery system. This component also helps the water penetration into the systems thus the erosion and the diffusion of the active agent.

Kolliphor RH40 (KP) was used as a wetting agent. This is a polyoxyethylene 40 castor oil derivative and can be used as an emulsifying or wetting agent. Literature data shows that it has a congealing temperature between 16 and $26^{\circ} \mathrm{C}$, and a melting point approximately at $30{ }^{\circ} \mathrm{C}$ $[109,112]$.

\subsubsection{Materials used for the electrospun nanofibrous systems}

For the PLA-based fibers polylactic acid (PLA) (Ingeo 4032D type, NatureWorks LLC., Minnetonka, MN, USA), dichloromethane, dimethyl sulfoxide (Molar Chemicals Ltd., Halásztelek, Hungary) and metronidazole (Ph. Eur. 8., Hungaropharma Plc., Budapest, Hungary) were used.

Polylactic acid is an aliphatic polyester, which can be derived from renewable sources i.e. plants. In terms of physical properties, PLA can be crystalline, semi-crystalline or highly crystalline. Glass transition of this material is between 60 and $65^{\circ} \mathrm{C}$ and melting point is 130 $180{ }^{\circ} \mathrm{C}$.

PLA is often referred to as a bioplastic because it is a biodegradable polymer, and in the medical field, it is used to create absorbable sutures, bioabsorbable implants for orthopedics and systems for controlled drug delivery of active agents [113]. To create the nanofibers PLA (Ingeo 4032D type, NatureWorks LLC., Minnetonka, MN, USA) was used.

\subsection{Methods}

\subsubsection{Preparation of lipid-based systems}

All the formulations were created by a melting and homogenation method. Firstly, the two lipophilic components (CA and SBP) and the surfactant (KP) were melted together at $70{ }^{\circ} \mathrm{C}$ on a hot plate, then the polymer (ZnHA or Methocel E4M) was suspended and homogenized with an overhead stirrer at 50 RPM. The components of drug free compositions for the preformulation studies can be seen in the Table 2 . 
Table 2. Composition of formulations analyzed during the preformulation studies of lipid-based delivery systems.

\begin{tabular}{ccccc}
\hline \multicolumn{5}{c}{ Concentration of the components $(\mathbf{m} / \mathbf{m} \%)$} \\
$\begin{array}{c}\text { Structure-building } \\
\text { component }\end{array}$ & Lipid base & Surfactant & Polymer \\
\hline WB & CA & SBP & KP & $\begin{array}{c}\text { Methocel } \\
\text { E4M }\end{array}$ \\
\hline 10 & 0 & 85 & 5 & 0 \\
\hline 10 & 0 & 80 & 10 & 0 \\
\hline 10 & 0 & 75 & 15 & 0 \\
\hline 10 & 0 & 70 & 20 & 0 \\
\hline 0 & 5 & 90 & 5 & 0 \\
\hline 0 & 5 & 85 & 10 & 0 \\
\hline 0 & 5 & 80 & 15 & 0 \\
\hline 0 & 5 & 75 & 20 & 0 \\
\hline 20 & 0 & 67 & 10 & 3 \\
\hline 40 & 0 & 47 & 10 & 3 \\
\hline 60 & 0 & 27 & 10 & 3 \\
\hline 0 & 20 & 67 & 10 & 3 \\
\hline 0 & 40 & 47 & 10 & 3 \\
\hline 0 & 60 & 27 & 10 & 3 \\
\hline
\end{tabular}

In the case of the formulation studies for the drug delivery sytems, the antimicrobial agent(s) (AMX, MZ, ZnGlu) was/were dispersed and homogenized at $50^{\circ} \mathrm{C}$ to avoid thermal decomposition. Delivery systems were created by molding the melted formulations into round shaped silicone molds. Cylindrical drug delivery systems were $1.5 \mathrm{~mm}$ in thickness and $9 \mathrm{~mm}$ in diameter.

In Table 3, the composition of the drug delivery systems is shown. A unique code has been assigned to each individual formulation $[109,114]$.

Table 3. The composition of lipid-based delivery systems.

\begin{tabular}{cccccccc}
\hline & \multicolumn{7}{c}{ Composition of delivery systems $(\mathbf{m} / \mathbf{m} \%)$} \\
\hline Components & F1 & F2 & F3 & F4 & F5 & F6 & F7 \\
\hline SBP & 32 & 32 & 32 & 47 & 46.8 & 32 & 42 \\
\hline CA & 40 & 40 & 40 & 40 & 40 & 40 & 40 \\
\hline KP & 10 & 10 & 10 & 10 & 10 & 10 & 10 \\
\hline Methocel E4M & 3 & 3 & 3 & - & - & - & 3 \\
\hline MZ & - & 15 & 7.5 & - & - & 15 & - \\
\hline ZnHA & - & - & - & 3 & 3 & 3 & - \\
\hline AMX & 15 & - & 7.5 & - & - & - & - \\
\hline ZnGlu & - & - & - & - & 0.2 & - & - \\
\hline $\mathbf{2 \%}$ CHX solution & & & & & & & 5 \\
\hline
\end{tabular}




\subsubsection{Preparation of PLA-based nanofibrous drug delivery systems}

PLA-based systems were produced at the Budapest University of Technology and Economics in the Laboratory of Plastics and Rubber Technology. The production parameters were briefly the following: fibers were spun at ambient temperature at $15 \mathrm{kV}$ voltage, $10 \mathrm{~cm}$ collector distance and a feeding rate of $3 \mathrm{~cm}^{3} / \mathrm{h}$, from the $80 / 20 \mathrm{v} / \mathrm{v} \%$ mixture of dichloromethane and dimethyl sulfoxide. The solution contained the polymer in $10 \mathrm{~m} / \mathrm{m} \%$, while the amount of the active component was changed from 0 to $3 \mathrm{~m} / \mathrm{m} \%$ of the solution.

The fibers were investigated as native fibers and as compressed disks. Neat electrospun fiber mats were taken directly from the aluminum collector film and round-shaped disks were obtained by compressing approximately $10-15 \mathrm{mg}$ of the neat fiber under $1 \mathrm{kN}$ pressure for $30 \mathrm{~s}$ in a pellet die of $13-\mathrm{mm}$ diameter.

\subsubsection{Investigation of drug delivery systems}

\subsubsection{Optical contact angle measurements}

The behavior of the drug delivery systems in contact with aqueous media was studied with an OCA Contact Angle System (Dataphysics OCA 20, Dataphysics Inc., GmbH, Germany). The contact angle of water droplets was determined. The measurement was done with a water droplet of $10 \mu \mathrm{L}$ volume, which was dropped from a calibrated syringe onto the surface of the various delivery systems.

In the case of lipid-based delivery systems, contact angles were measured immediately after contact. Five parallel measurements were performed on each sample.

PLA-based native fibers and compressed disks were measured in a similar way, but contact angles of droplets were measured immediately after, and 10 and 20 minutes subsequent to droplet placement, as well. Five parallel measurements were carried out.

\subsubsection{Differential scanning calorimetry of lipid-based systems}

The softening temperature and the melting point of the lipid formulations were determined by differential scanning calorimetry measurements with a Mettler-Toledo DSC 821e instrument in $\operatorname{argon}$ atmosphere $(100 \mathrm{~mL} / \mathrm{min})$. The temperature was raised from $+5{ }^{\circ} \mathrm{C}$ to $+100{ }^{\circ} \mathrm{C}$ by 5 ${ }^{\circ} \mathrm{C}$ per minute. $10 \mathrm{mg}$ of the samples were put in $40 \mu \mathrm{L}$ aluminum pans. The tops were holed, then the pans were sealed.

Two sets of measurements were carried out: during the preformulation studies formulations without active agents were analyzed, while during the characterization of API containing 
formulations, the effect of the incorporated active agent on the melting point and softening temperature was investigated.

\subsubsection{X-ray diffraction (XRD) measurements}

The solid state of the components was analyzed by means XRD measurements. A Bruker D8 Advance diffractometer (Bruker AXS GmbH, Karlsruhe, Germany) with $\mathrm{CuK} \alpha$ radiation $(\lambda=1.5406 \AA)$ was used for the XRD analysis. The samples were scanned at $40 \mathrm{kV}$ and $20 \mathrm{~mA}$ from $3^{\circ}$ to $40^{\circ} 2 \theta$ angle at a scanning rate of $0.1^{\circ} / \mathrm{s}$ and a step size of $0.01^{\circ}$.

\subsubsection{Hardness of lipid-based systems}

The hardness of lipid-based formulations was investigated by a TA.XT Plus C texture analyzer. During the measurements, a probe $50 \mathrm{~mm}$ in diameter penetrated $0.5 \mathrm{~mm}$ into the formulations. The evaluation was carried out in different circumstances: at room temperature $\left(25^{\circ} \mathrm{C}\right)$ and after 10 minutes of immersion into $1 \mathrm{~mL}$ of water at body temperature $\left(37^{\circ} \mathrm{C}\right)$. Three parallel measurements were carried out.

\subsubsection{In vitro drug release tests}

\subsection{Lipid-based systems}

The in vitro drug release profiles of the delivery systems were determined. The compositions were weighed with an analytical scale, put in 50-mm-long dialysis tubes (Spectra/Por ${ }^{\circledR}$ Standard RC tubing, MWCO: 12-14 kD) and sealed with closures. The tubes containing the formulations were placed in $7.5 \mathrm{~mL}$ of PBS solution (prepared by dissolving $8 \mathrm{~g} / \mathrm{dm}^{3} \mathrm{NaCl}, 0.2$ $\mathrm{g} / \mathrm{dm}^{3} \mathrm{KCl}, 1.44 \mathrm{~g} / \mathrm{dm}^{3} \mathrm{Na}_{2} \mathrm{HPO}_{4} \cdot 2 \mathrm{H}_{2} \mathrm{O}$ and $0.12 \mathrm{~g} / \mathrm{dm}^{3} \mathrm{KH}_{2} \mathrm{PO}_{4}$ in distilled water and the $\mathrm{pH}$ was adjusted to 7.4 by adding an adequate amount of $0.1 \mathrm{M} \mathrm{HCl}$ ) thermostated at $37{ }^{\circ} \mathrm{C}$. Drug release was investigated for seven days, and three parallel measurements were carried out. One milliliter of sample was taken (at $0.5,1,2,4,6,10,24,30,48,72,96$ and $168 \mathrm{~h}$ ) and replaced with $1 \mathrm{~mL}$ of fresh PBS solution thermostated at $37^{\circ} \mathrm{C}$.

CHX was quantified by UV spectrophotometric analysis at $250 \mathrm{~nm}$, while AMX and MZ were analyzed with Merck-Hitachi LaChrome Elite HPLC (Hitachi High Technologies America, Inc., Schaumburg, IL, USA) using UV detector at $230 \mathrm{~nm}$. The column was a Kinetex $250 \mathrm{~mm} \times 4.6 \mathrm{~mm}$ column packed with $53 \mu \mathrm{m}$ EVOLuna C18, $100 \AA$ (Phenomenex Inc., Torrance, CA, USA). Isocratic elution was performed with 20:80 (v/v) Methanol- $\mathrm{NaH}_{2} \mathrm{PO}_{4}$ $(0.05 \mathrm{M})$ at a flow rate of $1 \mathrm{~mL} / \mathrm{min}$. Retention time for AMX and MZ was at 4.6 and 6.7 minutes, respectively. The resolution $\left(\mathrm{R}_{\mathrm{S}}\right)$ was 3.10 . 


\subsection{PLA-based nanofibers}

The in vitro drug release profiles of PLA nanofibers were determined by the measurement of dissolution followed by UV-Vis spectroscopy (Helios $\alpha$ Thermospectronic UVspectrophotometer v4.55, Unicam: Thermo Fisher Scientific, Waltham, MA) at $318 \mathrm{~nm}$. Disks $(0.014-0.017 \mathrm{~g})$ and neat fiber mats $(0.012-0.016 \mathrm{~g})$ were weighed and put into $7.5 \mathrm{~mL}$ of $p \mathrm{H}=7.4 \mathrm{PBS}$ solution thermostated at $37^{\circ} \mathrm{C}$. Samples of $1.0 \mathrm{~mL}$ volume were taken at $0.5,1$, 4, 6, 10, 24, 30, 48, 72, 96 and 196 hours and replaced with $1.0 \mathrm{~mL}$ of fresh PBS solution. Drug release was followed for 7 days.

\subsubsection{Antimicrobial effectiveness of formulations}

Typical periodonthopathogenic bacteria, namely F. nucleatum (ATCC 25586), Parvimonas micra (ATCC 33270), Eikenella corrodens (ATCC 23834), P. gingivalis (ATCC 33277), A. actinomycetemcomitans (ATCC 29524) and Prevotella intermedia (clinical isolate no. 118710) control strains were used. (In the case of nanofibrous drug delivery systems the measurements with $P$. gingivalis could not be carried out, because the previously used control strain showed no sign of growth, and no other control strain was available.) A 1 McFarland standard concentration bacterial suspension of each bacterial strain was made separately with $0.9 \% \mathrm{NaCl}$ solution (in suspension it is equivalent to approximately $3 \times 10^{8}$ colony forming units $/ \mathrm{mL}$ ). The suspension was spread onto a horse blood agar plate, where then formulations were placed on. After 24 hours of incubation in anaerobic conditions (Concept 400 anaerobic incubator, Biotrace International Plc., UK), the diameter of the inhibition zones was measured. The formulations were then put on a new horse blood agar plate, also inoculated with 1 McFarland standard concentration freshly made bacterial suspension of each of the above-mentioned bacterial strains. The plates were then put in an anaerobic chamber for 24 hours. This was repeated until no inhibition zone could be detected.

In our microbiological investigations, the antimicrobial activity of our formulations was measured on six different strains of oral pathogenic bacteria which may contribute to the initiation of periodontitis. As mentioned above, these were the following: E. corrodens, $P$. intermedia, P. micra, F. nucleatum, A. actinomycetemcomitans and $P$. gingivalis.

E. corrodens - a facultative anaerobic, Gram-negative bacterium - is a human (mostly oral) pathogen. E. corrodens appears to be susceptible to beta-lactam antibiotics, but resistant to $\mathrm{MZ}$ [115].

P. micra (previously known as Peptostreptococcus micros or Micromonas micros) - an anaerobic, Gram-positive coccus - is the member of the normal human gastrointestinal flora 
[116]. According to Rams et al., it shows high susceptibility to penicillin, but MZ seems to be less effective against the bacterium [117].

$P$. intermedia - a Gram-negative anaerobic bacterium with black pigments - is often connected with oral and subgingival diseases. $P$. intermedia shows susceptibility to beta-lactam antibiotics and MZ [118].

F. nucleatum - a predominantly oral and periodontal anaerobic pathogen - can be linked to a variety of human diseases. Strains isolated from endodontic infections seem to be highly sensitive to AMX and to a lesser extent to $\mathrm{MZ}[119,120]$.

A. actinomycetemcomitans - a Gram-negative, facultatively anaerobic rod - is frequently associated with most forms of periodontitis and numerous oral infections. AMX is effective against the bacterium, while it shows much less susceptibility to $\operatorname{MZ}[121,122]$.

$P$. gingivalis - an obligately anaerobic, Gram-negative black-pigmented rod - is the most commonly linked microorganism to periodontal disease. According to susceptibility tests, $P$. gingivalis is susceptible to AMX and MZ [123,124].

\section{RESULTS AND DISCUSSION}

\subsection{Lipid-based systems}

The aim of this part of my thesis was to create a local, swellable, degradable drug delivery system with the following requirements: a) to soften but not totally melt at body temperature; b) to accommodate to the shape of the periodontal pocket; c) to have proper wetting properties in order to aqueous media be able to access the incorporated materials; d) to swell in an aqueous environment, which contribute to the erosion and elimination of the delivery systems; e) to have a mucoadhesive feature; f) to be effective against microorganisms responsible for periodontal disease; and g) to provide extended drug release [109,114].

The approach to the satisfaction of these requirements was to formulate a hydrophobic matrix which has a lipid base, contains a structure-building component, a polymer, and a surface-active agent. Proper choice of these excipients could provide the delivery systems with other features such as swelling, erosion, or has a soft but coherent structure.

Incorporation of hydrophilic antimicrobial agents into a hydrophobic matrix could protect the antimicrobial agents and hinder penetration of aqueous media into the devices, and, thus, allowing sustained release of drugs.

According to the abovementioned requirements, the following components were selected. A lipid base with a melting point at body temperature (Suppocire BP (SBP)) was chosen. 
Structure-building components were selected to prevent total melting of formulations (white beeswax (WB) and cetostearyl alcohol (CA)). A surface-active agent was picked to help the wetting of formulations (Kolliphor RH40 (KP)). Different polymers were used to aid water penetration into the formulations, and swelling and erosion of lipid systems, and to provide a mucoadhesive feature to formulations (Methocel E4M and zinc hyaluronate (ZnHA)). Antimicrobial agents were incorporated into the systems to provide antibiotic effect (amoxicillin (AMX), metronidazole (MZ), chlorhexidine gluconate (CHX), zinc gluconate (ZnGlu) and zinc hyaluronate (ZnHA)).

The abovementioned active agents were used alone (AMX, MZ, CHX or ZnHA) or in combination with another agent. Formulations were created with the combination of AMX and MZ; ZnHA and ZnGlu; and ZnHA and MZ. The delivery systems into which ZnHA was incorporated, did not contain HPMC, because ZnHA also possesses mucoadhesive features.

\subsubsection{Preformulation study}

During the preformulation study, different measurements were carried out to determine the suitable concentration and the type of the structure-building component. The concentration of the surface-active agent and the polymer were also determined.

DSC measurements were used to analyze the effect of different structure-building components and their concentration. The effect of surface-active agent concentration on the melting point of the formulations was analyzed, as well.

Wettability of formulations was also investigated to confirm the chosen concentration of the surfactant and the polymer. The wetting of the formulation with the surrounding media is crucial for the future drug release; therefore, it is an effort to provide an ideal contact angle between the system and the aqueous medium.

Cetostearyl alcohol was chosen as a structure-building component, as formulations containing white beeswax - independent of the concentration of white beeswax - disintegrated after one day of immersion in water at body temperature, which could result in a too fast drug release.

DSC measurements indicated that the best softening range of formulations may be achieved if the concentration of cetostearyl alcohol is fixed at $40 \mathrm{~m} / \mathrm{m} \%$. The concentration of the surface-active agent did not influence the softening and melting point of formulations.

By wettability measurements the concentration of the surface-active agent was determined. Best wettability of formulations could be achieved if the concentration of the surface-active agent was at $10 \mathrm{~m} / \mathrm{m} \%$. 
The concentration of the polymers was determined by wettability measurements as well. Results indicated that $3 \mathrm{~m} / \mathrm{m} \%$ of Methocel E4M and $3 \mathrm{~m} / \mathrm{m} \%$ of ZnHA is suitable for the delivery systems.

The concentration of SBP - the lipid base - and the active agent together was always held at $47 \mathrm{~m} / \mathrm{m} \%$. The concentration of APIs was either determined by a preliminary antimicrobial investigation or literature data analysis.

To conclude the results of the preformulation study, CA was chosen as the structure-building component. Its concentration was held at $40 \mathrm{~m} / \mathrm{m} \%$. The concentration of $\mathrm{KP}$ - the surfaceactive agent - was determined to be $10 \mathrm{~m} / \mathrm{m} \% .3 \mathrm{~m} / \mathrm{m} \%$ of polymers were incorporated into the delivery systems. The sum of the concentration of the lipid-base and the antimicrobial agent was $47 \mathrm{~m} / \mathrm{m} \%$.

Formulations shown in Table 2 were the drug delivery systems of which further evaluation were carried out.

\subsubsection{Evaluation of lipid drug delivery systems}

Further evaluation of delivery systems with incorporated active agents were carried out to analyze the effect of the incorporated antimicrobials on the melting point and softening temperature of formulations. The state of the APIs was also investigated along with the consistency of delivery systems and the possibility of sustained drug release. Effectiveness of formulations against bacteria was also determined.

\subsubsection{Differential scanning calorimetry of formulations}

DSC method was employed to analyze if the incorporated active agents modify the softening temperature or melting point of the delivery systems. DSC curves of the formulations can be found in Figure 4.

The lipid base of the compositions was SBP which has a melting point at $39.08{ }^{\circ} \mathrm{C}$ according to our measurements. CA, which was used as the structure-building component of the formulations, had a melting point at $61{ }^{\circ} \mathrm{C}$. The results of DSC measurements show that $\mathrm{KP}$ has a congealing temperature approximately between $20-30^{\circ} \mathrm{C}$, which corresponds with the literature data. No sharp peaks could be perceived on the curves belonging to the polymers (ZnHA and Methocel E4M), and zinc gluconate in this temperature range $\left(10-100{ }^{\circ} \mathrm{C}\right)$. 


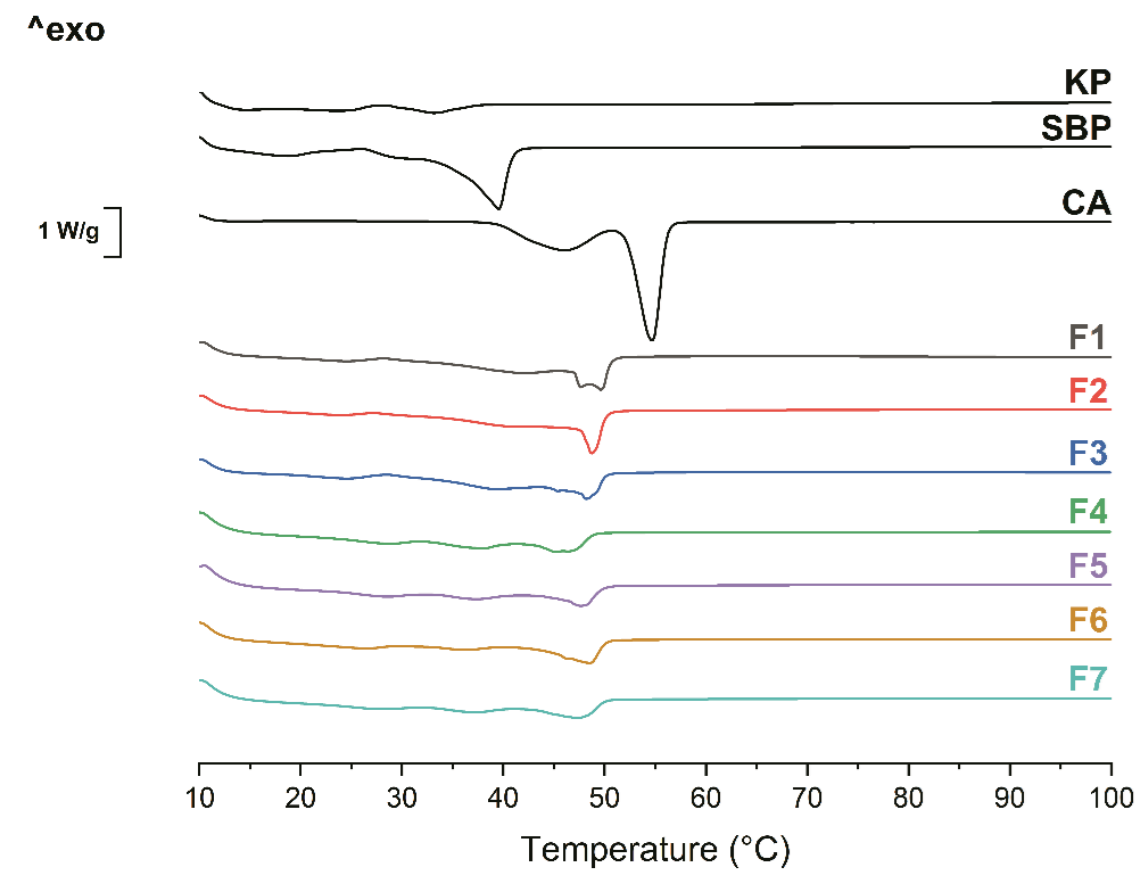

Figure 4. DSC curves of bases and different formulations F1-F7.

Formulations F1-F7 were examined to investigate the possible modifying effect of the incorporated drugs and excipients on melting point. Antibiotics or other suspended materials may partially dissolve in the lipid base, therefore, the effect of various incorporated components of different amounts on the melting point was also investigated. In all formulations, the sharp peaks of SBP and CA disappeared, even the two peaks of CA morphed into one, and shifted to temperatures between the melting points of the two pure components. At approximately $30{ }^{\circ} \mathrm{C}$, a moderate melting can be observed, but total melting only occurs between 40 and $50{ }^{\circ} \mathrm{C}$. This moderate melting supports the softening of the systems, but, the presence of components with melting points at about $50{ }^{\circ} \mathrm{C}$ suggests the existence of a coherent structure at body temperature.

By the thorough analysis of the DSC results, it could be concluded that no peaks of any curve belonging to different formulations could be associated with the decomposition of any component, thus, the constituents are expected to be stable in this temperature range. Moreover, incorporated active agents did not influence the melting point highly, and the softening temperature of the formulations in the preformulation studies are held.

\subsubsection{Evaluation of hardness}

Measurement of the hardness of formulation F7 (CHX) was carried out in order to analyze the deformability and consistency of the drug delivery systems in different circumstances (at room temperature in a dry state; and at body temperature in an aqueous environment which imitates the insertion into the periodontal pockets). In this texture analysis, a spherical probe 
with a diameter of $5 \mathrm{~mm}$ penetrated $0.5 \mathrm{~mm}$ into the formulations. This means that the force is measured which is needed for the approximate $50 \%$ deformation of the delivery systems.

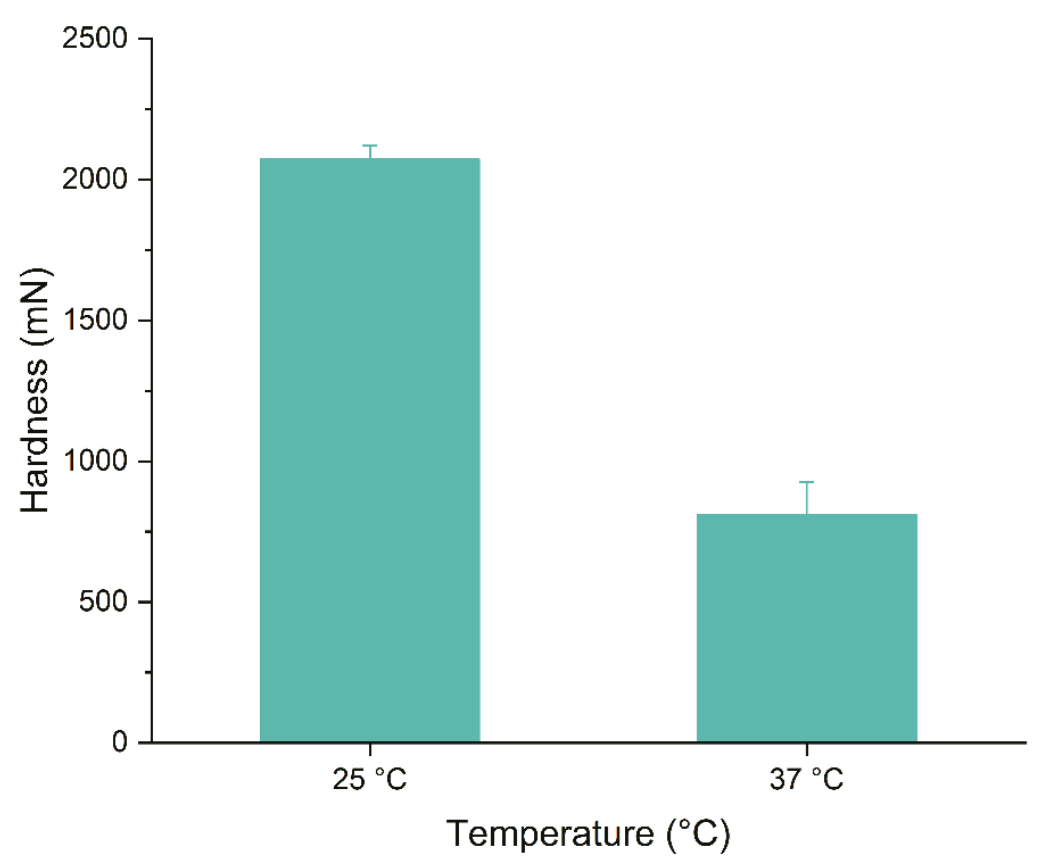

Figure 5. Hardness of formulation F7 in different conditions: at $25^{\circ} \mathrm{C}$ in a dry state and at $37^{\circ} \mathrm{C}$ in aqueous media.

According to the results displayed in Figure 5, $2074 \pm 48 \mathrm{mN}$ force is needed to deform the delivery systems at room temperature. When the formulations were held at $37^{\circ} \mathrm{C}$ in aqueous media, this force dropped down to $810 \pm 116 \mathrm{mN}$.

The decrease of force needed to deform the delivery systems indicates that the structure suffered a considerable softening, but coherent forces of the structure-building component did not cease entirely. Results are in conjunction with the results of DSC analysis and confirm that total melting of formulations do not occur at body temperature; therefore, the delivery systems can stay at the site of infection (at the subgingival area), can accommodate to the shape of the periodontal pockets (PP) and can provide sustained release of antimicrobial drugs.

\subsubsection{X-ray diffraction analysis}

XRD analyses were carried out to investigate the state of the antibiotic drugs. The presence of solid crystals is indicated by sharp peaks in the diffractograms, while molecularly dispersed or amorphous components, as they do not possess a crystalline structure, are not shown. The amorphous structure is often a substantial feature of polymers; thus, a crystalline structure is absent and characteristic diffractograms can rarely be obtained. 


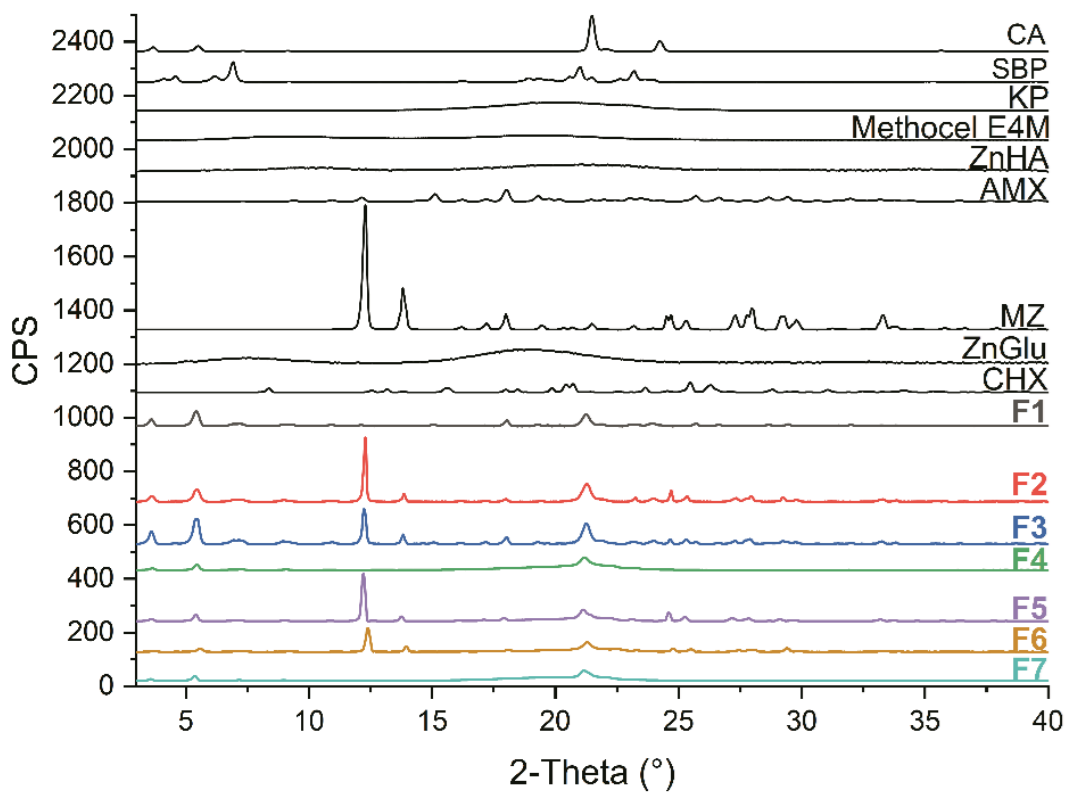

Figure 6. Diffractogram of pure components (Kolliphor RH40 (KP), Suppocire BP (SBP) and cetostearyl alcohol (CA), amoxicillin (AMX), metronidazole (MZ), zinc hyaluronate (ZnHA), zinc gluconate (ZnGlu), Methocel E4M, and chlorhexidine $\mathrm{CHX}$ ) and formulations $\mathrm{F} 1-\mathrm{F} 7$.

All of the components, except HPMC, KP, ZnHA, and ZnGlu, have a crystalline structure according to the XRD diffractograms presented in Figure 6.

SBP and CA have similar molecular structures, which will result in analogous diffractograms. This means that in the diffractograms of the formulations containing all of the components, it is hard to differentiate between the two lipid components, SBP and CA during the evaluation of the different formulations. The more intensive peak of CA could overlap with and cover the peak of SBP at the same $2 \theta$ value in the preparations. This peak, which belongs to $\mathrm{CA}$, has decreased in intensity in the diffractograms of the formulations, which implies that the crystallinity of CA has suffered a setback, which is in accordance with the DSC results, where the two peaks of pure CA have fused together and shifted to a lower temperature range.

A characteristic peak linked to SBP at the $2 \theta$ value of approximately $5^{\circ}$ is present in all formulations with varied intensity.

The characteristic peaks of AMX and MZ could be separated from those of the lipid components. The presence of characteristic peaks in the diffractograms is the sign of solid particles in the formulations. AMX and MZ have a characteristic peak at the $2 \theta$ value of $18.1^{\circ}$ and $12.3^{\circ}$, respectively. Peaks of AMX or MZ at the mentioned $2 \theta$ values could be observed in the diffractograms of formulations F1 (AMX), F2 (MZ), F3 (AMX+MZ) and F6 $(\mathrm{MZ}+\mathrm{ZnHA})$. 
The presence of ZnHA or ZnGlu in the preparations could not be demonstrated by this experiment because of their amorphous structure, to which no characteristic peaks belong in an XRD diffractogram.

Interestingly, characteristic peaks of CHX cannot be seen in the diffractogram of F7 (CHX). There may be more, than one explanation to this phenomenon. Firstly, CHX may be dissolved in the lipid base of the delivery systems. Secondly, CHX may be still dissolved in the aqueous phase which is emulsified with the lipid components. Thirdly, CHX may have precipitated from the aqueous solution - when it was added to the melted lipid components -and the precipitated crystals are suspended in the lipid base. However, the concentration of crystalline CHX is below the limit of detection of the applied method.

The partially crystalline form of the lipid matrix (CA and SBP) could contribute to the coherent structure of the system, which does not melt at body temperature, thus providing sustained release of antibiotic compounds.

\subsubsection{Drug diffusion from the delivery systems}

Drug dissolution measurements were carried out in order to evaluate the drug release profiles of the prepared formulations which contain MZ (F2, F3 and F6) or CHX (F7). Delivery systems were put into dialysis tubes, and MZ was quantified by an HPLC method, whereas CHX was quantified by UV-Vis spectrophotometry.

The amount of released $\mathrm{MZ}$ is shown in Figure 7. Formulations F2 (MZ) and F6 (MZ+ZnHA) have shown similar drug release profiles. A plateau phase commences at approximately the 100th hour in both cases, which is in accordance with a total drug release.

However, the drug release curves of formulation F3 (AMX+MZ) indicates that only onethird of the amount was liberated from the preparations despite that half of the amount had been incorporated into the compositions.

$\mathrm{MZ}$, which is a more soluble material in water compared to AMX, could be liberated more quickly from formulations creating capillaries, which contribute to a higher swelling and therefore allow water to access all the suspended drug in the delivery systems. On the contrary, when smaller amounts of MZ are incorporated into the delivery systems with AMX - a substance with lower water solubility - fewer capillaries may be formed during drug release, permitting less water to penetrate the systems and resulting in non-complete drug dissolution.

The application of ZnHA did not change the release profile, which can be explained by the similar swelling and degradation profiles of formulations F2 (MZ) and F6 (MZ+ZnHA). 


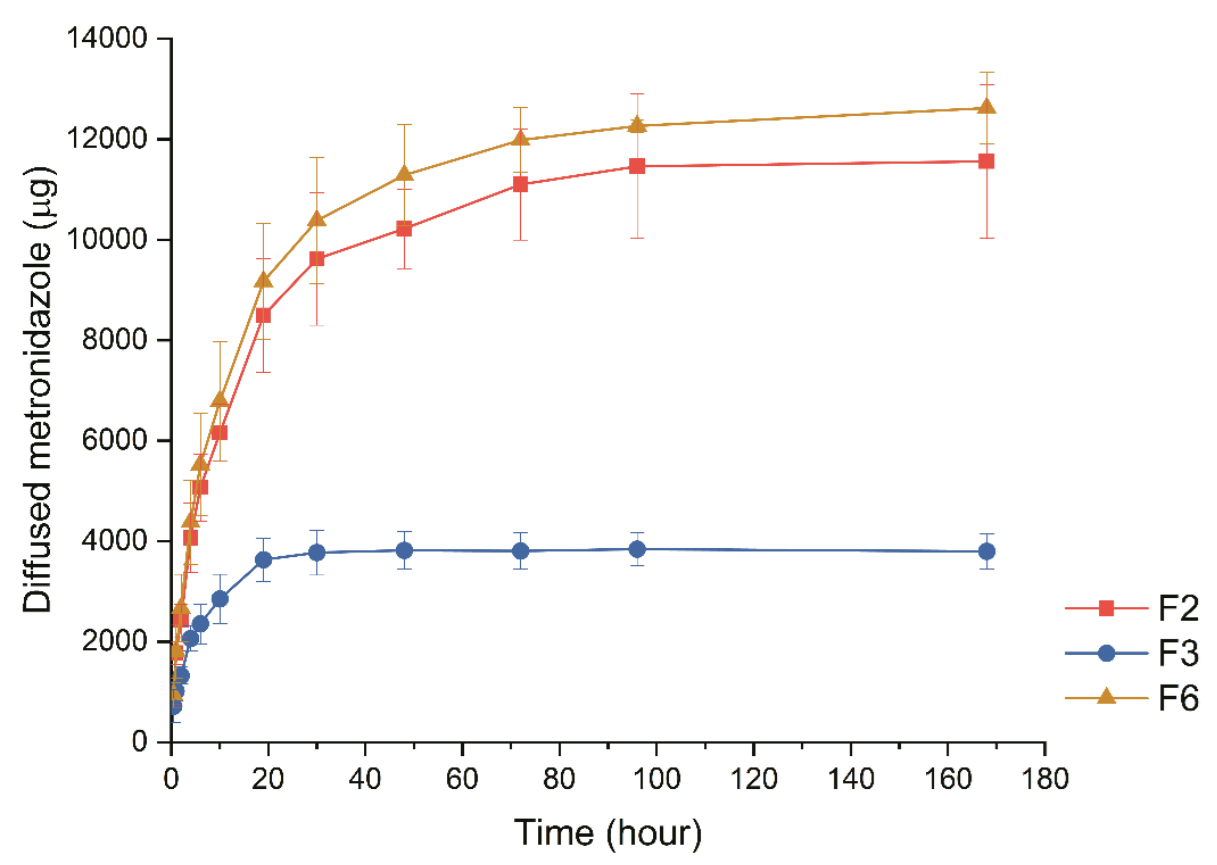

Figure 7. The amount of metronidazole diffused from formulations F2, F3, and F6.

In the case of formulation F7 (CHX) during the one-week (170.5 hour) investigation period, approximately $225 \mu \mathrm{g}$ of active agent diffused from the delivery systems, which is nearly $50 \%$ of all incorporated drug (Figure 8). On average, 19\% (83 $\mu \mathrm{g}$ ) of all incorporated CHX diffused from the delivery systems during the first 24-hour period. A plateau phase could not be observed during the test length, which indicates that the release continues after 1 week.

According to Figure 8., the amount of drug diffused from formulation F7 during one week is approximately $50 \%$ of all incorporated drug, which indicates sustained release. Bakó and colleagues investigated the diffusion of CHX from PerioChip ${ }^{\circledR}$ [125], which is also a CHXcontaining drug delivery system, in different $p \mathrm{H}$ conditions. Comparing their results in $p \mathrm{H} 7.4$ PBS solution to the results of our in vitro drug diffusion measurements, it can be concluded that formulation F7 provides a continuous release, while in the case of PerioChip ${ }^{\circledR}$ a burst release can be observed (40\% of drug released during 24 hours). This burst release is followed by a slow release which results in $27 \%$ more released drug at the end of the 1 -week observation period. This indicates that our formulated CHX containing lipid-based systems can provide a continuous release at a higher but steady rate.

Summarizing the results of drug diffusion testing, a sustained release of drugs could be achieved with these compositions, and the main factors affecting drug release are swelling (driven by the applied hydrophilic components such as polymer, active ingredients and their concentration) and the strength of the coherent lipid structure. 


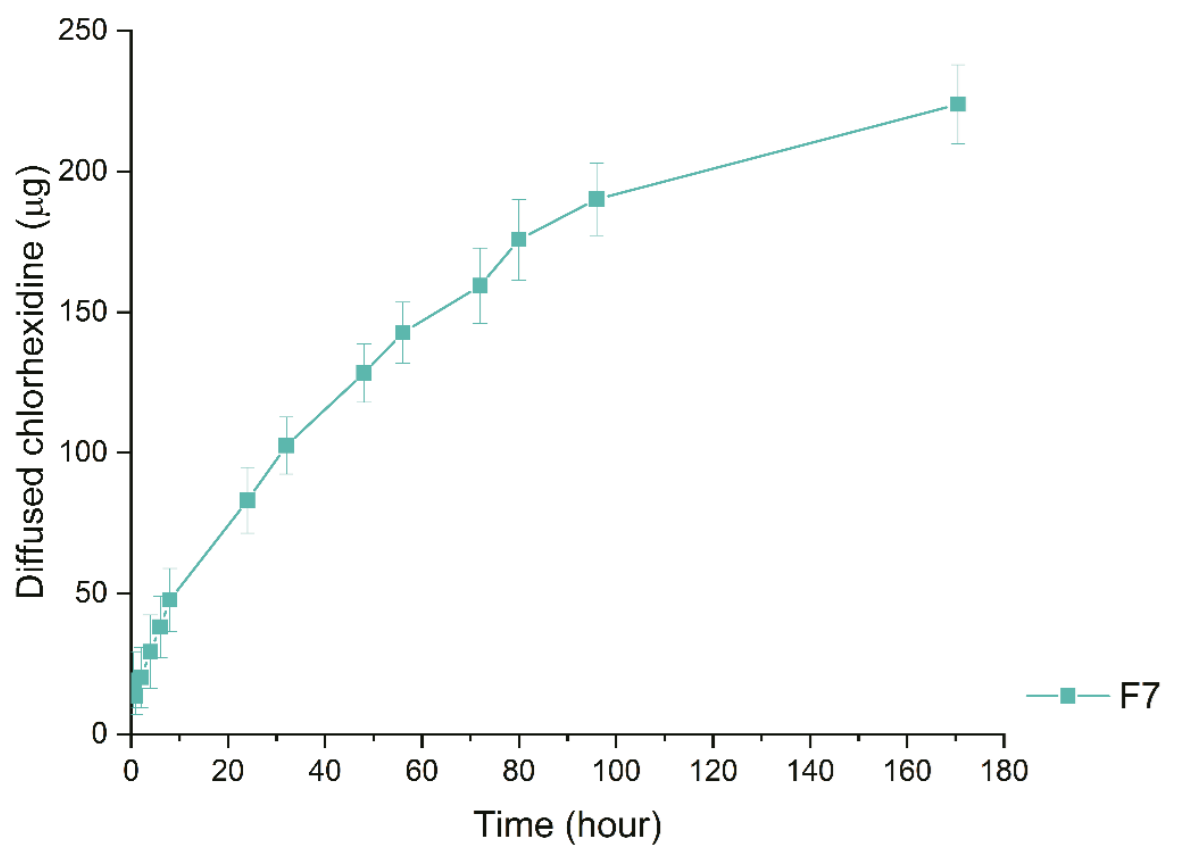

Figure 8. The amount of chlorhexidine diffused from formulation F7.

\subsubsection{Antimicrobial effectiveness of formulations}

Numerous different species of microorganisms may be present in the oral cavity or on the dental surfaces. The composition of the oral microbiota varies widely from person to person, from place to place and naturally with dietary habits [126,127].

According to Kolenbrander and colleagues [128], these species can be separated into two categories concerning plaque formation. Species in the first category (initial colonizers) are thought to stick to the tooth surface and proliferate. The second group (late colonizers) binds to the first group of bacteria via different interactions.

Hyaluronic acid (HA) is a naturally occurring polysaccharide of the extracellular matrix of connective tissue, synovial fluid, and soft periodontal tissues as well. Its application in the treatment of the inflammatory process is established in different medical areas such as orthopedics, dermatology, and ophthalmology. In the treatment of periodontal diseases, hyaluronic acid shows anti-inflammatory effect [129], whereas the zinc salt of HA possesses an antimicrobial effect [114].

Zinc salts may have a beneficial effect when used in mouth rinses, can possibly decrease plaque formation by inhibiting glycolytic enzymes and may prevent the attachment of bacteria 
to the tooth surface [130]. These salts administered in combination with other antimicrobial agents may show a synergism by means of antibacterial effect [131].

In our microbiological investigations, the inhibition zone of the formulation was measured using fresh agar plates day-by day. The length of the inhibition was evaluated in case of the different formulations, the results can be found in Figure 9.

According to our measurements, P. micra was the most sensitive microorganism to AMX containing formulations as the compositions could provide 18 days of effective drug release, while the least susceptible pathogen was E. corrodens with only 9 days of growth inhibition.

MZ susceptibility was slightly lower, as the effect against the most sensitive bacterium $P$. gingivalis was only nine days. E. corrodens was the least susceptible to $\mathrm{MZ}$ as on the first 2 days no growth could be detected, but after two days there was no inhibition zone around the formulation. This result is in accordance with the literature data, where it was established that E. corrodens is resistant to MZ [132].

Formulation F3 (AMX+MZ) did not provide larger inhibition zones than formulations containing only one antimicrobial agent as the antimicrobial effect lasted for a shorter time. $P$. gingivalis was an exception: this bacterial strain was the only one with higher susceptibility to the combination of AMX and MZ.

In most cases, the growth inhibition effect of combinations lasted longer than that of MZ. This could have been possible due to the more potent antimicrobial effect of AMX. Lower susceptibility to the combination of AMX and MZ compared to only AMX containing formulations may be due to the decreased concentration of AMX in the formulation F3 $(\mathrm{AMX}+\mathrm{MZ})$ and the lower susceptibility of bacteria to MZ.

Susceptibility to ZnHA is different among various bacterial strains. $A$. actinomycetemcomitans and E. corrodens show resistance to ZnHA, but combined with ZnGlu, a longer effect can be observed in case of A. actinomycetemcomitans. E. corrodens remains unsusceptible to the combination of $\mathrm{ZnHA}$ and $\mathrm{ZnGlu}$.

According to the results, the same susceptibility characterizes $P$. gingivalis and $P$. intermedia when using $\mathrm{ZnHA}$ alone or in combination with $\mathrm{ZnGlu}$. In case of $P$. micra and F. nucleatum, the results show that higher efficiency may be achieved by administering a combination of ZnHA and ZnGlu instead of using only ZnHA. 


\section{A. actinomycetemcomitans}

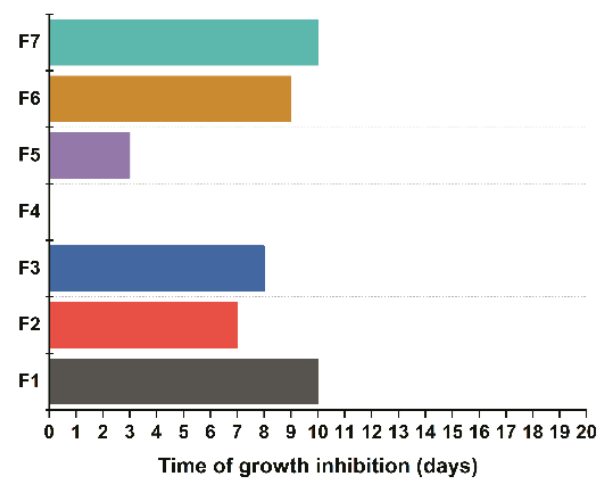

\section{F. nucleatum}

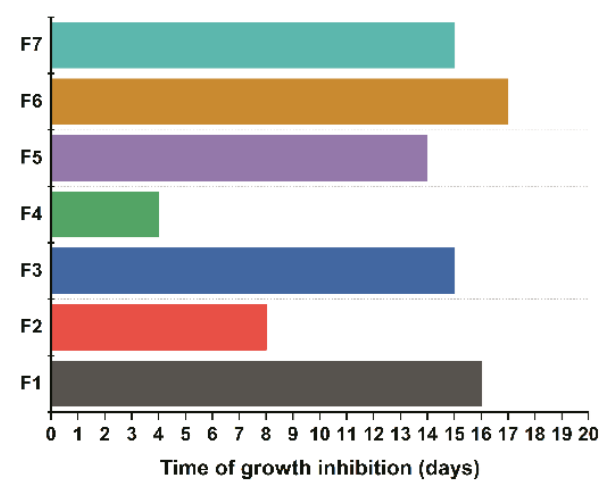

\section{P. gingivalis}

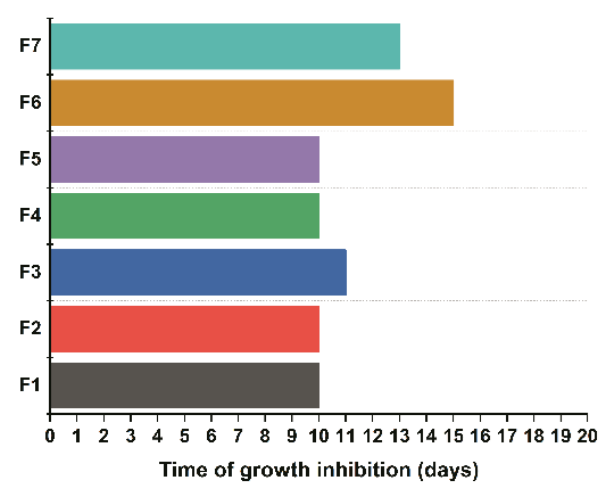

\section{E. corrodens}

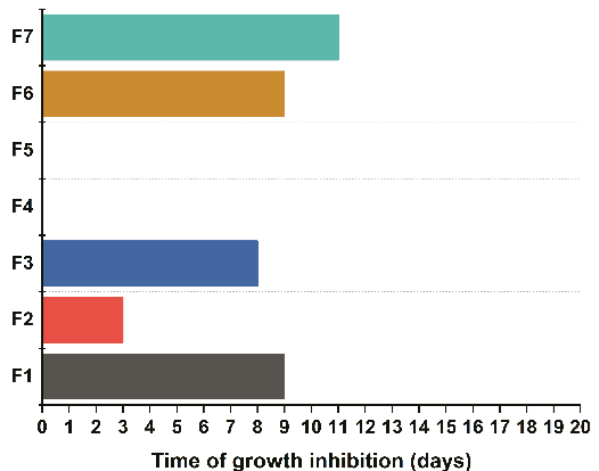

P. micra

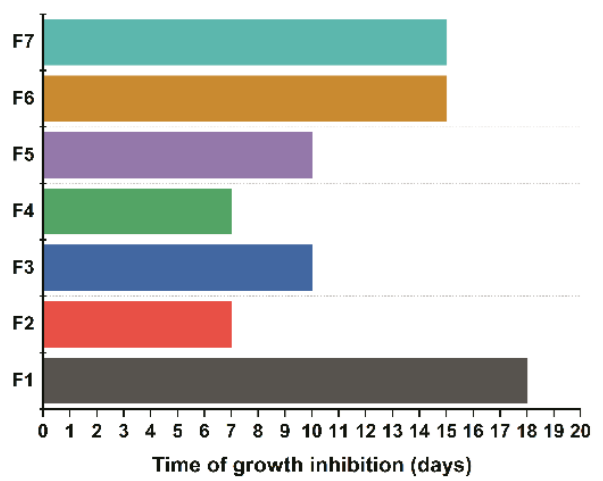

P. intermedia

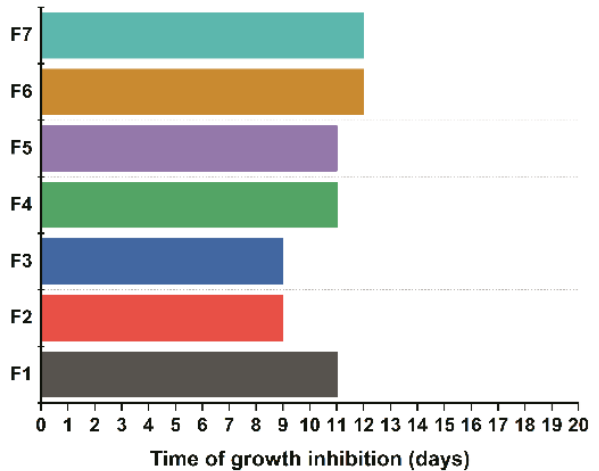

Figure 9. Time of bacterial growth inhibition of formulations F1-F7.

The results imply that the combination of $\mathrm{MZ}$ and $\mathrm{ZnHA}$ provides a synergistic effect: formulations containing both $\mathrm{ZnHA}$ and $\mathrm{MZ}$ provided longer growth inhibition against the microorganisms than the active agents alone. The synergistic effect is most conspicuous against E. corrodens where formulations with $\mathrm{MZ}$ only having a two-day inhibitory effect, and $\mathrm{ZnHA}$ had no antibacterial effect against the bacteria, while the combination showed a nine-day effect.

The outcome of the antimicrobial investigation of ZnHA supports that alone it may not be effective enough, but a combination with an antimicrobial drug(s) (e.g., MZ) may provide a synergistic and - in this case - a longer positive effect. 
Formulation F7 (CHX) has effectively inhibited the growth of all used bacteria. The least susceptible strain was A. actinomycetemcomitans (10 days of growth inhibition), while the most susceptible was $F$. nucleatum and P. micra (15 days of growth inhibition). These results indicate why is CHX is still used in the everyday dental practice.

All in all, it can be concluded that formulations F1 (AMX), F6 (MZ+ZnHA) and F7 (CHX) were the most effective against these six bacterial strains. ZnHA alone may not be effective, but in combination with ZnGlu they may be active against susceptible bacteria. In contrast with literature data, the combination of AMX and MZ did not provide the expected effectiveness. Formulation F2 (MZ) has also showed mild growth inhibition but with formulation F6 (CHX) longer inhibition of bacterial growth could be achieved.

\subsubsection{Summary of the formulated lipid-based drug delivery systems}

To summarize the first part of my experimental work, it can be concluded that:

- By using DSC and OCA measurements, the optimal composition of the lipid-based systems and the proper structure-building component was identified during the preformulation study: $40 \mathrm{~m} / \mathrm{m} \% \mathrm{CA}, 10 \mathrm{~m} / \mathrm{m} \% \mathrm{KP}, 3 \mathrm{~m} / \mathrm{m} \%$ polymer (ZnHA or HPMC) were chosen. The sum of the concentration of SBP and the incorporated active agent is fixed at $43 \mathrm{~m} / \mathrm{m} \%$. This composition is able to provide a softening at body temperature and sustained release of incorporated materials.

- DSC investigation of drug loaded formulations indicated that incorporation of active ingredients does not influence highly the softening and melting point of the formulations.

- XRD measurements proved that the incorporated materials are mostly suspended in crystalline form in the lipid base.

- Swelling and erosion of formulations were confirmed during my $\mathrm{PhD}$ work [114]. Swelling and erosion profiles depend on the incorporated materials, their attributes and the coherent lipid structure.

- In vitro drug release measurements confirmed that sustained drug release of incorporated active agents is possible.

- Antimicrobial effectiveness investigations revealed that the formulations provide long-term effectiveness against anaerobic pathogen bacteria responsible for the initiation of the disease. 
- The investigation of antimicrobial effect on anaerobic bacteria showed that the highest effectiveness can be reached by the incorporation of AMX, CHX or the combination of $\mathrm{MZ}$ and $\mathrm{ZnHA}$.

\subsection{Electrospun drug delivery systems}

\subsubsection{Sample preparation}

The MZ concentration of the fibers was 12.2 and $25.7 \mathrm{~m} / \mathrm{m} \%$, the latter corresponding to the MZ concentration of the saturated spinning solution. Neat electrospun fiber mats taken directly from the aluminum collector film and round-shaped, compressed disks were obtained by compressing approximately $10-15 \mathrm{mg}$ of the neat fiber under $1 \mathrm{kN}$ pressure for 30 seconds in a pellet die of $13 \mathrm{~mm}$ diameter. Samples are shown in the pictures in Figure 10.

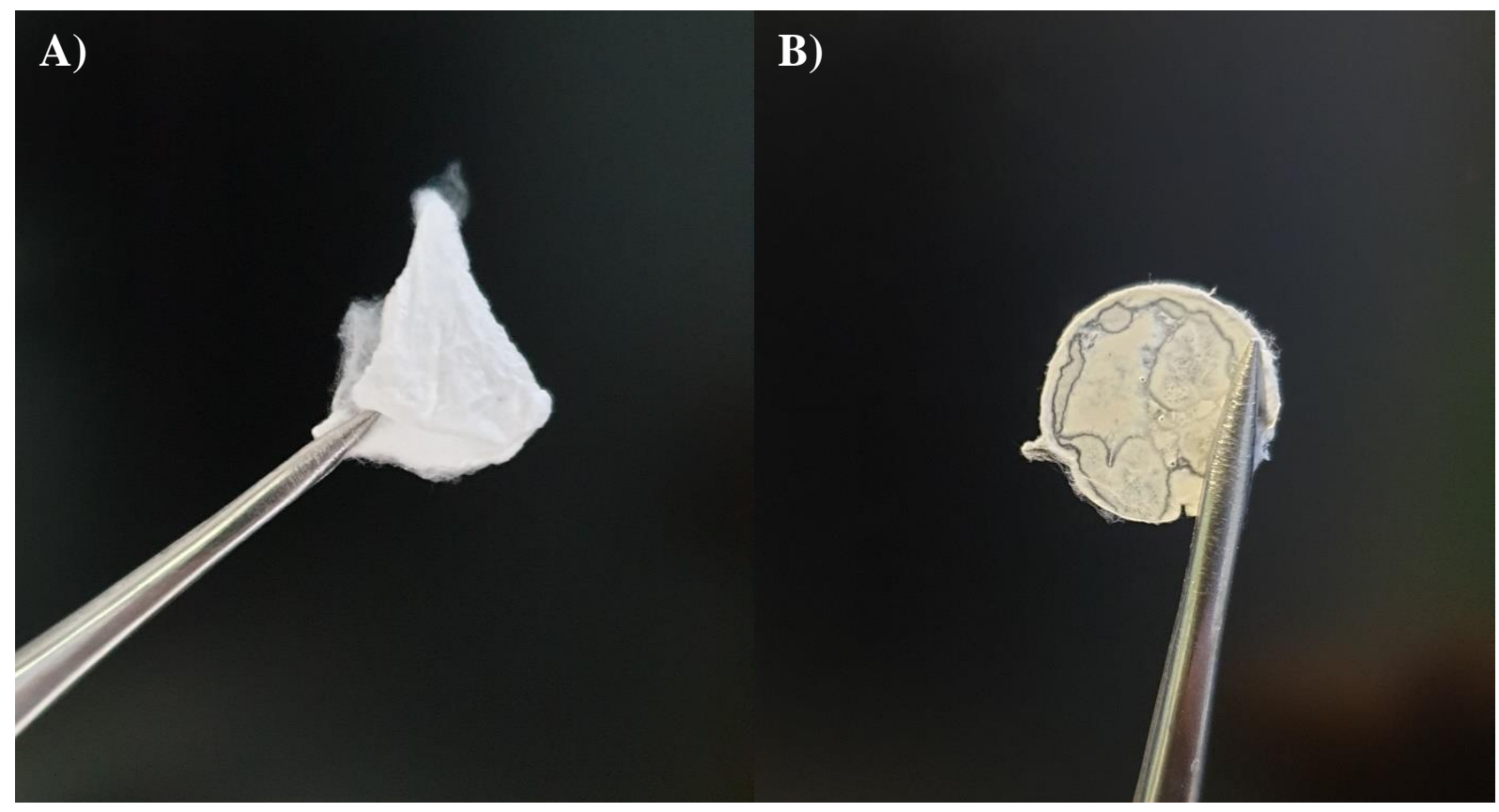

Figure 10. Neat fibers (A) directly from the collector and compressed disks (B) obtained by compression of neat fibers.

\subsubsection{Scanning electron microscopy}

The structure of the fiber mat and the disks was also studied by scanning electron microscopy. Micrographs showing the differences in structure are presented in Figure 11. The mat consists of loose fibers with considerable space among individual fibers (Figure $11 \mathrm{~A}$ ). One would expect fast penetration and easy flow of the fluid used for dissolution and thus very fast release of the drug. The scrutiny of micrographs recorded on mats reveals the presence of MZ crystals among the fibers. The structure of a disk is shown in the micrograph of Figure $11 \mathrm{~B}$ ). The disk has a much more compact structure, voids are smaller, and the fibers are close to each other. The presence of MZ crystals among the fibers is more obvious in this case. The SEM 
study proves that a part of the drug is distributed in crystal form among and probably also within the fibers.

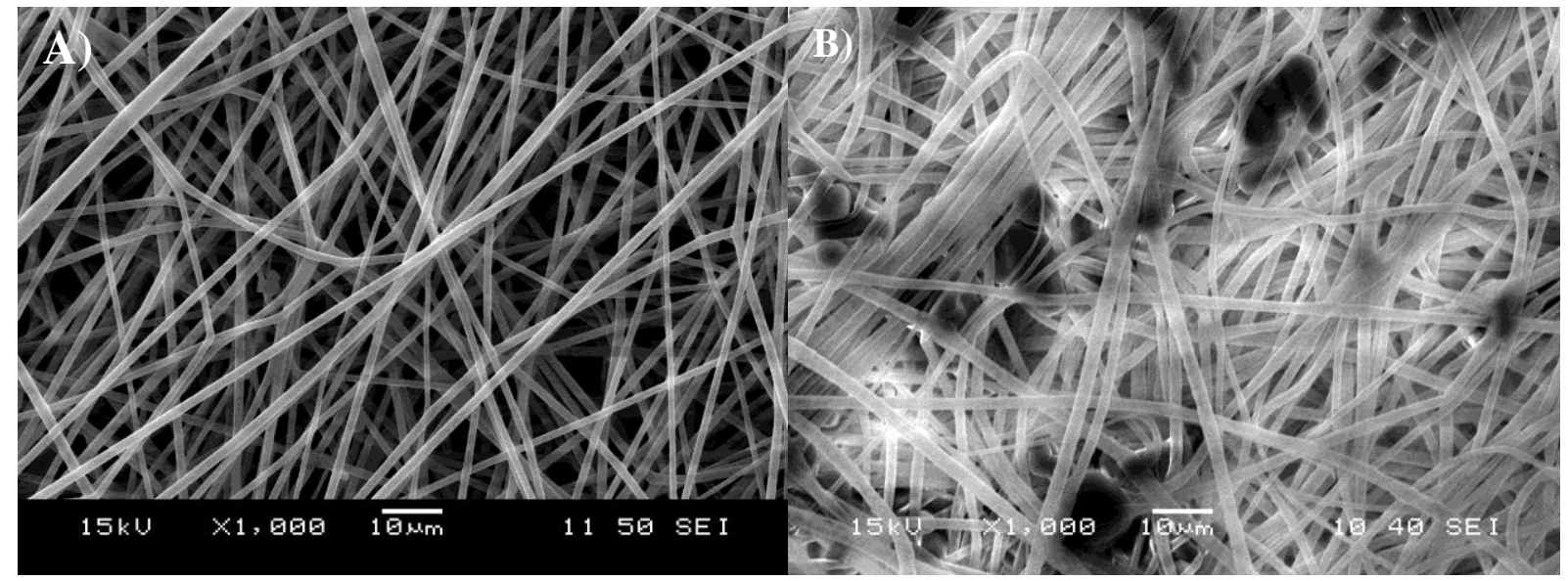

Figure 11. SEM micrographs recorded on the PLA devices studied. A) fiber mat, B) compressed disk. The black dots are crystalline metronidazole particles in picture B).

\subsubsection{X-ray diffraction analysis}

XRD analyses of native and pressed samples prior to and after dissolution testing were carried out to investigate the crystalline structure of the incorporated MZ and the PLA fibers.

MZ-containing and blank devices were analyzed. Both native and compressed fibers were included in the investigation. Diffractograms are shown in Figure 12.

Characteristic peaks of MZ appear in the diffractograms of all metronidazol-containing fibers at approx. 13.8 and $14.92 \theta$, only the intensity varies with the concentration.

Diffractograms of PLA fibers without incorporated API show that electrospun PLA filaments have a semi-crystalline structure ( $2 \theta$ of peak is approx. 16.5), which was held and was more expressed (higher peaks and broader areas) when $\mathrm{MZ}$ was added to the systems. However, a decreased peak area and intensity could be observed after the dissolution test, indicating a decrease in the crystallinity of the semi-crystalline structure of the polymer.

The intensity of characteristic peaks belonging to $\mathrm{MZ}$ decreases during the dissolution experiment as an effect of drug release. It can also be seen that some MZ remains in the device even after the dissolution experiment, which indicates that some of the drug precipitates during fiber spinning and crystals are located not only among, but also within the fibers. The release of this latter part of the drug is quite slow and does not take place in the time scale of the dissolution experiment. 


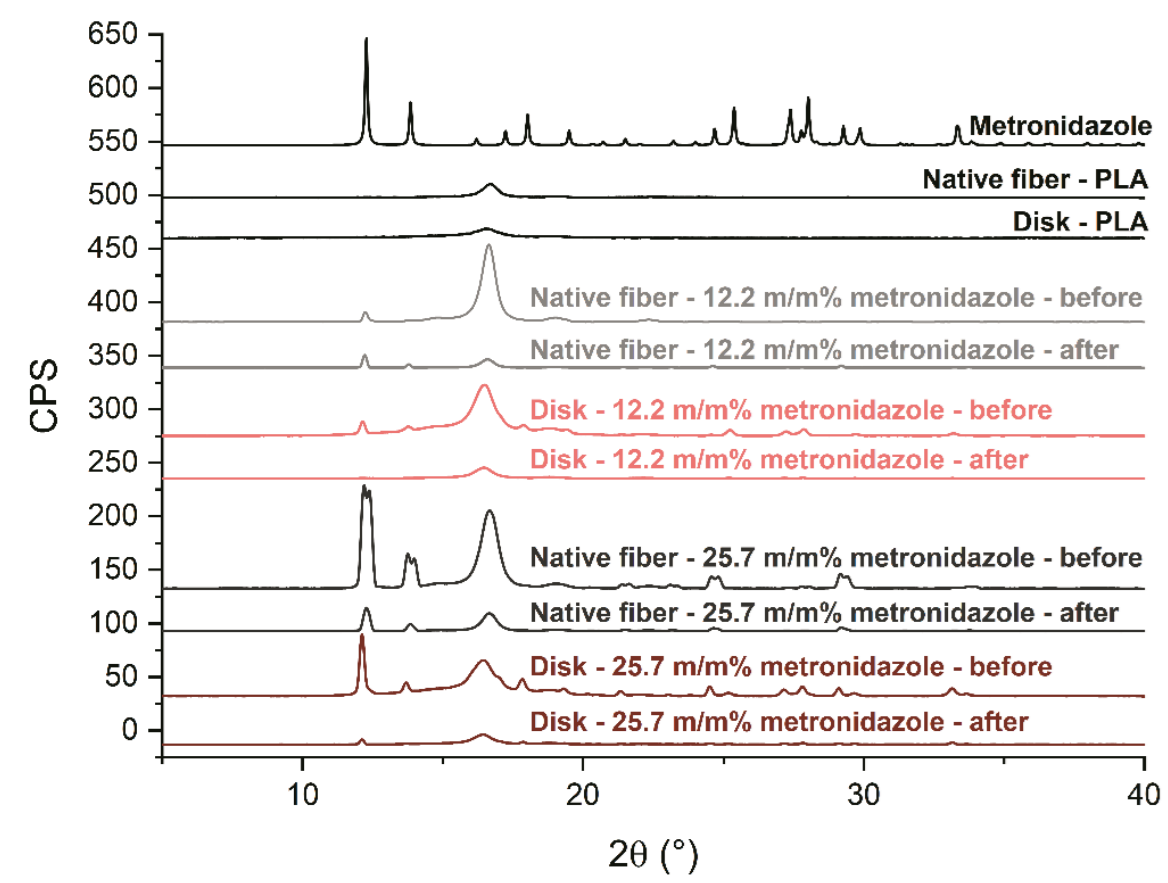

Figure 12. XRD diffractograms of native fibers and compressed disks before and after in vitro drug release measurements.

\subsubsection{Wettability analysis}

In order to gain insight related to the penetration of the dissolution medium into the devices prepared, the contact angle of water was measured on the formulated native fibers, and the compressed disk and MZ as a function of time. The results are presented in Table 4. Contact angle is measured by placing a droplet of a liquid onto a smooth, stable surface. MZ dissolves in water, while both the fiber mats and the compressed disks have rough, porous surfaces into which the liquid may penetrate. Accordingly, the contact angle measured depends on the rate of dissolution in the case of MZ, and on surface tension and surface morphology in the case of the two devices. Pore size and capillary forces also play an important role in the formation of contact angle, but also in the rate of drug release. Since the surface tension of the components is constant and we do not expect large changes in the surface tension of PLA as an effect of the dissolution of some $\mathrm{MZ}$ in it, the major factors in the development of contact angle must be morphology, porosity and capillary forces.

According to the results Table 4, the contact angle of water on the MZ pastille is small, which is not surprising because MZ dissolves in water. Dissolution is confirmed by the fact that contact angle could not be measured after 10 minutes of forming the droplet on the surface. Contrary to MZ, the contact angle of the droplet placed onto the fiber mat is quite large, indicating poor wetting and penetration. Contact angle does not change with MZ content; the differences are caused by changes in morphology and the standard deviation of the 
measurements. Contact angles showed just a slight systematic decrease with time for the neat fiber mats, indicating that water can slowly penetrate into the mat.

Table 4 Contact angles measured (mean and SD values) on a metronidazole pastille and on drug release devices (fiber mats, disks) at various times.

\begin{tabular}{ccccc}
\hline \multirow{2}{*}{ Device } & $\begin{array}{c}\text { Drug content } \\
(\mathrm{m} / \mathrm{m} \%)\end{array}$ & \multicolumn{3}{c}{ Contact angle $\left({ }^{\circ}\right)$ after time $(\mathrm{min})$} \\
\cline { 2 - 5 } & 100 & 0 & 10 & 20 \\
\hline MZ pastille & 0 & $32.5 \pm 1.8$ & - & - \\
\hline \multirow{3}{*}{ Fiber mat } & 12.2 & $125.3 \pm 3.8$ & $123.2 \pm 4.0$ & $121.4 \pm 0.8$ \\
\cline { 2 - 5 } & 25.7 & $117.3 \pm 7.2$ & $112.3 \pm 9.5$ & $105.8 \pm 12.2$ \\
\cline { 2 - 5 } & 0 & $62.5 \pm 1.6$ & $48.7 \pm 0.5$ & - \\
\hline \multirow{3}{*}{ Disk } & 12.2 & $66.9 \pm 1.4$ & $46.4 \pm 0.3$ & - \\
& 25.7 & $64.3 \pm 0.5$ & $47.0 \pm 0.2$ & - \\
\cline { 2 - 5 } & & &
\end{tabular}

The contact angle measured on the compressed disks is much smaller and it is independent of the concentration of $\mathrm{MZ}$ as well. It decreases considerably with time and could not be measured after $10 \mathrm{~min}$ at all, showing that the water droplet placed onto the disk disappeared in this time interval. The difference in the contact angles measured on the fiber mat and the compressed disk proves that the primary factor determining aqueous media penetration is morphology and this factor is expected to determine the extent and rate of drug release as well.

The apparent contradiction that water cannot penetrate so fast into the loose fiber mats, while it does into the discs, should be considered. Capillary forces depend on the interaction of the liquid and the capillary and also on the size of the capillary, i.e. on pore size, in our case. The surface tension of water is $72 \mathrm{~mJ} / \mathrm{m}^{2}$ and that of PLA is around $40 \mathrm{~mJ} / \mathrm{m}^{2}$ [133-135]. Accordingly, water does not wet PLA fibers and cannot penetrate into the devices easily. Compression obviously changed the pore structure of the device, which decreases capillary forces and helps penetration. As an effect of changing morphology and penetration, we may expect faster release of the drug from the disk than from the fiber mat.

\subsubsection{Drug diffusion study}

The release of the drug incorporated into the devices prepared from electrospun fibers is a complex process and depends on several factors. As the results of the XRD measurements and the SEM micrographs showed, the drug, MZ in this case, is incorporated into the devices in various forms: as precipitated crystals within and among the fibers and as dissolved molecules in PLA. The dissolution of these forms must be different. The PBS solution must penetrate the device, dissolve the crystals and diffuse out into the surrounding medium. On the other hand, dissolved MZ must diffuse out of the PLA fibers into the surrounding medium. The solubility 
of MZ is small in PLA, and diffusion is driven by concentration difference, which is also small or even negative due to the large drug concentration of the surrounding solution because of the dissolution of the crystals. Consequently, the main factor determining the dissolution of MZ from the devices, i.e. drug release, is the penetration and flow of the PBS solution. This is different for the two devices, mats and disks, thus dissimilar drug release is expected from them.

The time dependence of dissolution is presented in Figure 13 for the fiber mats and the disks at two different $\mathrm{MZ}$ concentrations. In view of the observations presented, some of the results were expected. Compressed disks release the drug much faster than fiber mats because of the larger and faster penetration of the aqueous medium into the pores of the device. However, the fact that dissolution is independent of the initial concentration of MZ in the device is somewhat surprising. The fast penetration and dissolution of MZ in the PBS solution can result in the independence of concentration. The diffusion of the liquid, which contains the dissolved drug, into the surrounding medium may be the rate-determining step of dissolution in this case.

In the case of the fiber mats, the rate of dissolution depends on concentration, however, not as expected, i.e. higher rate at larger concentration, but in the opposite way. The slower release of the drug from the mats can be easily understood, if we consider the difference in the penetration of the aqueous medium. The effect of concentration, on the other hand, is difficult to explain. Obviously, the dissolution of the drug in the PBS solution and its diffusion into the surrounding medium are the rate-determining steps in this case. However, the presence of the drug did not influence diffusion rate much, thus dissolution must be dissimilar at the two concentrations of MZ. The larger drug concentration probably results in larger precipitated crystals, which leads to slower dissolution and release.

The rate of dissolution can be estimated from the data presented in Figure 13. According to this evaluation, plateau concentrations are reached after 24 hours for the disks, and after 48 or 96 hours for the fiber mats. However, time dependence can be evaluated quantitatively if appropriate functions are fitted to the experimental data. 


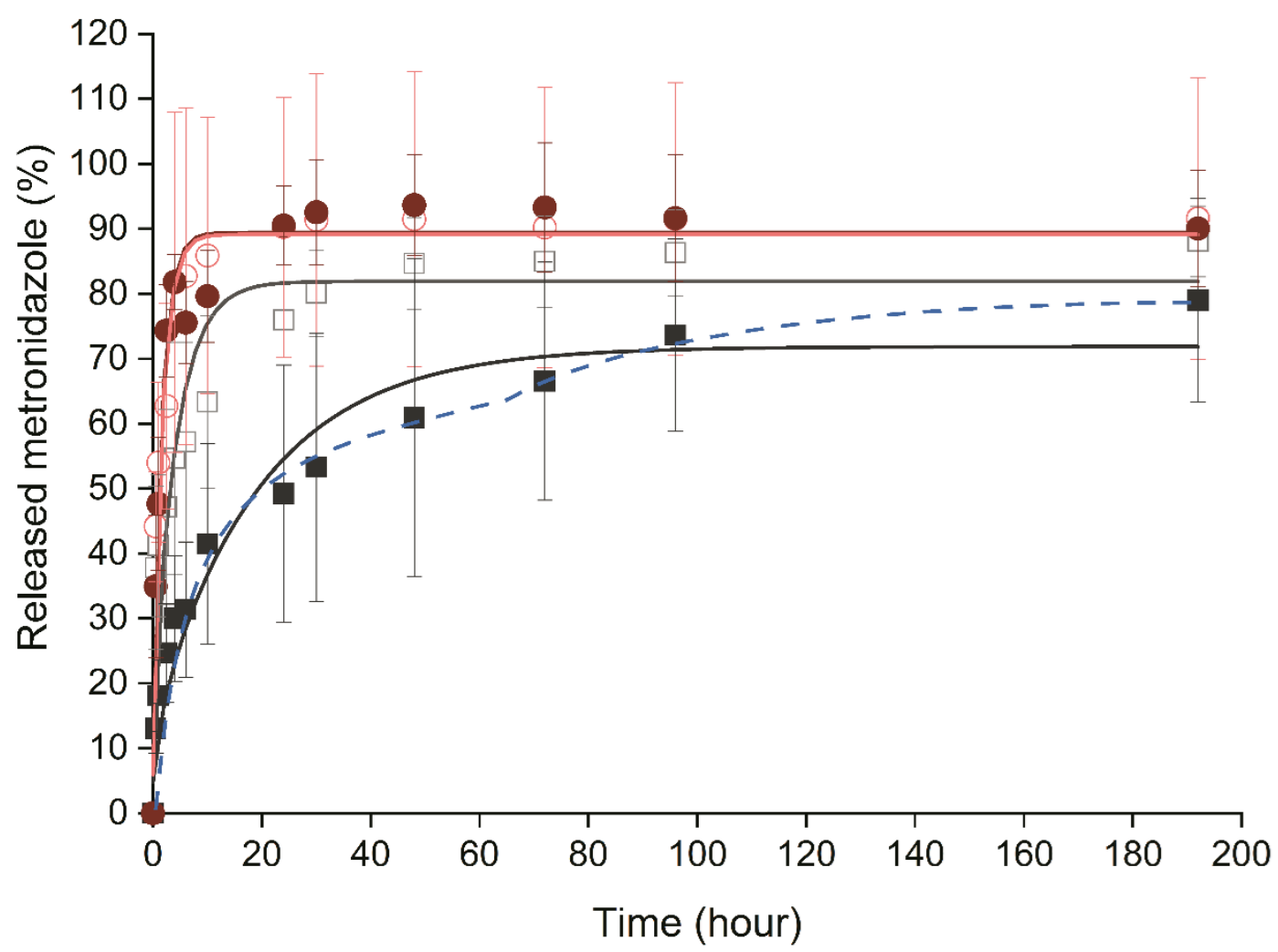

Figure 13. Dissolution of metronidazole from fiber mats and disks. Effect of drug content. Symbols: $(\square, \square)$ Fiber mats, $(\bigcirc$ Disks; empty symbols: 12.2 m/m\% MZ, full symbol: 25.7 m/m\% MZ.

The dissolution and the diffusion of the drug are determined by Fick's laws. Fick's equations can be solved numerically, or they can be expressed analytically using simplifications [136]. Two main approaches are used in practice, those describing the first part of the function plotting experimental results as the function of the square root of time, or those which use an exponential function. This approach gives a more accurate estimate at long times and it allows the estimation of the overall rate of dissolution and the maximum amount of dissolved material at infinite time (if the shape and structure of the device do not change, e.g.: fiber degradation). We followed the latter approach and fitted the function of Eq. 1 to the experimental results:

$$
M_{t}=M_{\infty}\left\{1-\frac{8}{\pi^{2}}\left[\exp (-a t)+\frac{1}{9} \exp (-9 a t)+\frac{1}{25} \exp (-25 a t)\right]\right\}
$$

where $\mathrm{M}_{\mathrm{t}}$ and $\mathrm{M}_{\infty}$ are the dissolved amount of drug at time $\mathrm{t}$ and at infinite time, respectively, and $\mathrm{a}$ is the overall rate of dissolution. The parameters calculated from the fitting are collected in Table 5. 
Table 5 Parameters characterizing the kinetics of dissolution determined by fitting Eq. 1 to the experimental results.

\begin{tabular}{ccccc}
\hline $\begin{array}{c}\text { Drug content } \\
(\mathrm{m} / \mathrm{m} \%)\end{array}$ & Form & $\mathrm{a}(1 / \mathrm{h})$ & $\mathrm{M}_{\infty}(\%)$ & $\mathrm{R}^{2}$ \\
\hline 12.2 & mat & 0.233 & 81.9 & 0.8855 \\
\hline 25.7 & mat & 0.051 & 71.8 & 0.9634 \\
\hline 12.2 & disk & 0.581 & 89.4 & 0.9449 \\
\hline 25.7 & disk & 0.556 & 89.1 & 0.9650 \\
\hline
\end{tabular}

Results presented in Table 5 confirm our qualitative evaluation and show that dissolution is much faster from the disk than from the fiber mat (see parameter a). It also confirms the composition dependence observed. The comparison of the predicted amount of drug dissolved at infinite time $\left(\mathrm{M}_{\infty}\right)$ indicates that a considerable amount of drug, 10-30\% remains in the devices after the dissolution experiment even in the case of the disks. Moreover, a closer comparison of the fitted lines and the measured values indicates that dissolution cannot be described with a single process; it consists of at least two steps, a rapid one at the beginning of the experiment and another one proceeding at a slower rate. This two-step process is demonstrated especially well by the results obtained on the fiber mat, containing $25.7 \mathrm{~m} / \mathrm{m} \%$ MZ. The two steps demonstrated by the blue broken line in the figure might be explained by the dissolution of the different forms of the drug; crystals located among the fibers dissolve much faster than dissolved MZ or crystals precipitated within the fibers. The different rates allow the regulation of the amount of the drug as a function of time and also the active lifetime of the device. Controlling the form of the drug in the device and the rate of water diffusion into the electrospun porous fiber network might be an efficient strategy to control drug release $[137,138]$.

\subsubsection{Antimicrobial effectiveness}

Disks were included in the microbiological study, as uniform shape, weight and thickness could not have been achieved with the fiber mats. The delivery systems evaluated contained 12.2 and $25.7 \mathrm{~m} / \mathrm{m} \%$ MZ. Five different bacterial strains were used in this investigation: $E$. corrodens, P. intermedia, P. micra, F. nucleatum, and A. actinomycetemcomitans.

The results of the microbiological study are presented in Figure 14. The duration of the antimicrobial effect is apparently independent of concentration; it is the same for disks with 12.2 and $25.7 \mathrm{~m} / \mathrm{m} \% \mathrm{MZ}$ content in most cases. A one-day difference appeared in growth inhibition for P. micra at 12.2 and $25.7 \mathrm{~m} / \mathrm{m} \% \mathrm{MZ}$ contents. In the other cases, disks with a larger MZ content provided slightly larger inhibition zones than systems containing less drug on most days. Bacterial growth inhibition is shorter, only $2-3$ days for $A$. 
actinomycetemcomitans, E. corrodens and $P$. micra, while the growth of $F$. nucleatum and $P$. intermedia was affected for a longer time, for 13 days.

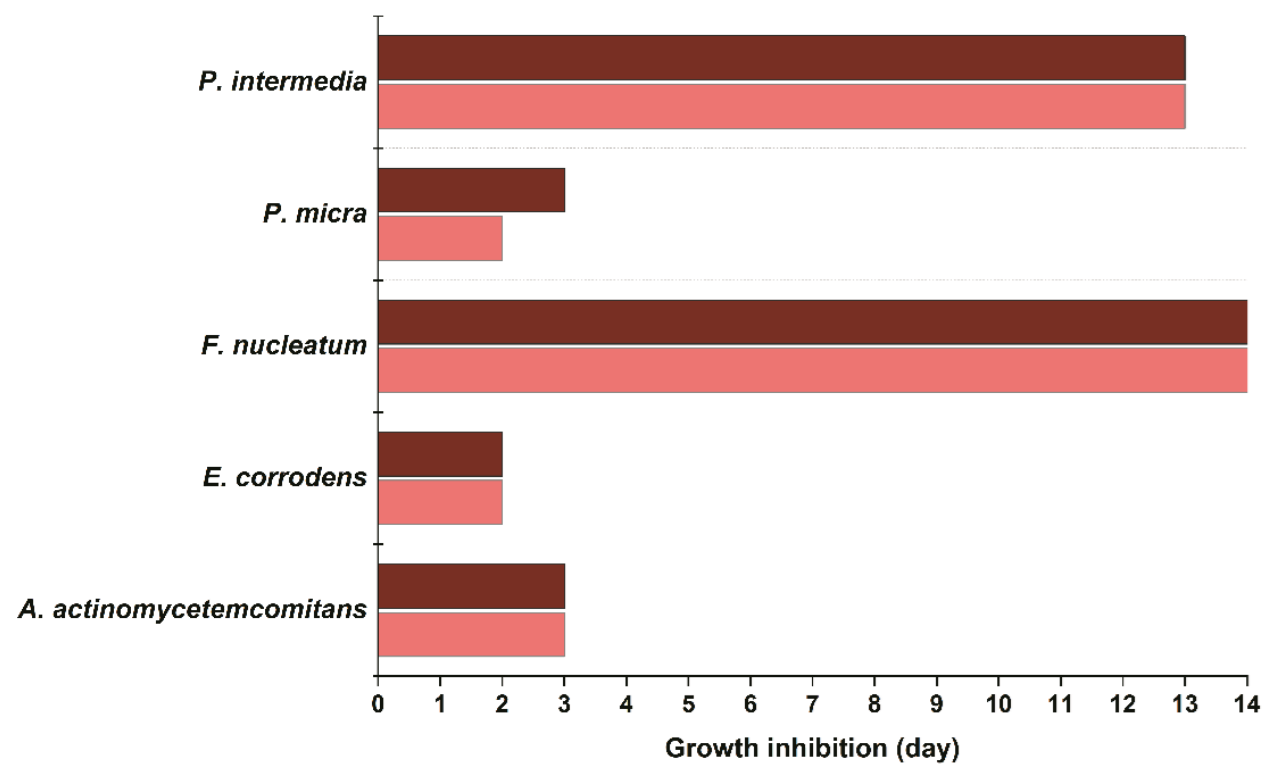

$12.2 \mathrm{~m} / \mathrm{m} \%$ metronidazole

$25.7 \mathrm{~m} / \mathrm{m} \%$ metronidazole

Figure 14. Inhibition of the growth of anaerobic pathogen bacteria. Time is shown in days.

The results of our measurements agree well with those published in the literature, suggesting that $F$. nucleatum and $P$. intermedia are more susceptible to $\mathrm{MZ}$ than $A$. actinomycetemcomitans, E. corrodens and P. micra, which may be completely resistant or minimally sensitive to the antimicrobial drug used. The diameter of the inhibition zone increases slightly with increasing MZ concentration, probably because of longer diffusion paths resulting in enhanced inhibition. The differences in the diameter of the inhibition zone are more pronounced for strains with larger susceptibility to $\mathrm{MZ}$ and they increase with time as well. Inhibition was observed in the growth of susceptible bacteria for as long as almost two weeks, indicating that our devices can be efficient for a long time. This fact, however, needs some consideration, because the dissolution study indicated that most of the drug is released from the disks in 24 hours. The contradiction might be explained by the difference in the conditions, but also in the presence of MZ located within the polymer in the form of dissolved molecules or precipitated crystals. The diffusion, thus the release rate of $\mathrm{MZ}$ is much slower in this latter case than for the drug located among the fibers in crystal form. A slower rate leads to prolonged inhibition times, which could result in greater patient compliance and better results of the periodontitis treatment. 


\subsubsection{Summary of formulated nanofiber systems}

In the second part of my work, an electrospun nanofibrous drug delivery system was created. 12.2 and $25.7 \mathrm{~m} / \mathrm{m} \%$ of MZ was incorporated. The delivery systems were further modified by applying pressure and, therefore, made into disks. Subsequent to the investigation of native fibers and compressed disks containing different amounts of MZ, the following conclusions were obtained:

- SEM investigations indicated that the compression process has not altered the fibers individually, but it has changed the capillary structure of the devices. Moreover, traces of MZ crystals among the fibers can be seen in the microscopic images.

- Results of XRD measurements confirmed that the majority of MZ is in crystalline form in both native fibers and compressed disks and PLA has a semi-crystalline structure. XRD diffractograms of devices after in vitro drug diffusion measurements shows traces of leftover MZ, which may refer to dissolved and crystallized MZ inside the filaments or MZ crystals which are inaccessible for the dissolution medium because of poor water penetration into the delivery systems.

- Results of wettability measurements indicated that water penetration into native fibers is slower than into disks.

- In vitro drug diffusion measurements showed that compression influences the drug diffusion from devices. MZ was released in 24 hours from disks, in 48 hours from native fibers containing $12.2 \mathrm{~m} / \mathrm{m} \% \mathrm{MZ}$ and in 96 hours from mats with $25.7 \mathrm{~m} / \mathrm{m} \%$ MZ.

- Investigation of antimicrobial effectiveness of disks indicates that disks may inhibit the growth of bacterial strains susceptible to $\mathrm{MZ}$ for 13 days.

\section{SUMMARY}

The aim of my Ph.D. work was the formulation and investigation of innovative drug delivery systems for the treatment of periodontal disease. Novelty of this work can be summarized as follows:

- The optimal composition of a local, swellable lipid-based drug delivery system was first described, which provides sustained release of incorporated antimicrobial agents and has a mucoadhesive feature. 
- The developed lipid formulation enables incorporation of high dose of different antimicrobials with an easy preparation method; moreover, the diffusion of the hydrophilic drugs could be prolonged up to 2 weeks.

- The synergism between the antimicrobial effect of ZnHA and MZ on 6 bacterial strains was proven by bacterial growth inhibition measurements.

- The wettability and water penetration into PLA-based nanofibrous devices were modified by compression, therefore the drug release was also altered.

All of the results of the measurements performed excellently illustrated the potential of the application of the developed lipid-based delivery system, its sustained release and high and long effectiveness against the growth of bacterial strains responsible for the disease. The effectiveness of PLA-based nanofiber disks was also shown, and results indicate that this device is suitable for the incorporation of antimicrobial materials. It was also proven that compression of nanofibers results in a device with uniform shape and size, which is convenient for the local treatment of periodontal disease.

In conclusion, all of the devices described in the thesis have the potential to be used in the treatment of periodontal disease. 


\section{REFERENCES}

1. Genco, R.J.; Borgnakke, W.S. Risk factors for periodontal disease. Periodontol. 2000 2013, 62, 59-94.

2. Tonetti, M.S.; Jepsen, S.; Jin, L.; Otomo-Corgel, J. Impact of the global burden of periodontal diseases on health, nutrition and wellbeing of mankind: A call for global action. J. Clin. Periodontol. 2017, 44, 456-462.

3. Chapple, I.L.C. Time to take periodontitis seriously. BMJ 2014, 348, g2645-g2645.

4. Jain, N.; Jain, G.K.; Javed, S.; Iqbal, Z.; Talegaonkar, S.; Ahmad, F.J.; Khar, R.K. Recent approaches for the treatment of periodontitis. Drug Discov. Today 2008, 13, 932-943.

5. Schwach-Abdellaoui, K. Local delivery of antimicrobial agents for the treatment of periodontal diseases. Eur. J. Pharm. Biopharm. 2000, 50, 83-99.

6. Southard, G.L.; Godowski, K.C. Subgingival controlled release of antimicrobial agents in the treatment of periodontal disease. Int. J. Antimicrob. Agents 1998, 9, 239-253.

7. Yar, M.; Farooq, A.; Shahzadi, L.; Khan, A.S.; Mahmood, N.; Rauf, A.; Chaudhry, A.A.; Rehman, I.U. Novel meloxicam releasing electrospun polymer/ceramic reinforced biodegradable membranes for periodontal regeneration applications. Mater. Sci. Eng. C 2016, 64, 148-156.

8. Do, M.P.; Neut, C.; Metz, H.; Delcourt, E.; Siepmann, J.; Mäder, K.; Siepmann, F. Mechanistic analysis of PLGA/HPMC-based in-situ forming implants for periodontitis treatment. Eur. J. Pharm. Biopharm. 2015, 94, 273-283.

9. Tyagi, P.; Vaish, S.; Dodwad, V. Clinical efficacy of subgingivally delivered $0.5 \%$ controlled release azithromycin gel in the management of chronic periodontitis. Indian J. Med. Sci. 2011, 65, 223-230.

10. Southard, G.L.; Godowski, K.C. Subgingival controlled release of antimicrobial agents in the treatment of periodontal disease. Int. J. Antimicrob. Agents 1998, 9, 239-253.

11. Yar, M.; Farooq, A.; Shahzadi, L.; Khan, A.S.; Mahmood, N.; Rauf, A.; Chaudhry, A.A.; Rehman, I. ur Novel meloxicam releasing electrospun polymer/ceramic reinforced biodegradable membranes for periodontal regeneration applications. Mater. Sci. Eng. C 2016, 64, 148-156.

12. Katancik, J.A.; Kumarswamy, A.; Branch-Mays, G.; Califano, J. V. Infections of the Periodontal Apparatus; Elsevier Inc., 2016;

13. Posnick, J.C. Periodontal Considerations in the Evaluation and Treatment of Dentofacial Deformities; Elsevier Inc., 2013; ISBN 9781455726981.

14. Cekici, A.; Kantarci, A.; Hasturk, H.; Van Dyke, T.E. Inflammatory and immune pathways in the pathogenesis of periodontal disease. Periodontol. 2000 2014, 64, 57-80.

15. Graziani, F.; Karapetsa, D.; Alonso, B.; Herrera, D. Nonsurgical and surgical treatment of periodontitis: how many options for one disease? Periodontol. 2000 2017, 75, 152188.

16. Tomasi, C.; Koutouzis, T.; Wennström, J.L. Locally Delivered Doxycycline as an Adjunct to Mechanical Debridement at Retreatment of Periodontal Pockets. $J$. Periodontol. 2008, 79, 431-439.

17. The webpage of the Smile First Family Dental Studio Available online: http://smilefirst.net/scaling-and-root-planing/ (accessed on Feb 18, 2020).

18. Heitz-Mayfield, L.J.A.; Lang, N.P. Surgical and nonsurgical periodontal therapy. Learned and unlearned concepts. Periodontol. 2000 2013, 62, 218-231.

19. Deas, D.E.; Moritz, A.J.; Sagun, R.S.; Gruwell, S.F.; Powell, C.A. Scaling and root planing vs. conservative surgery in the treatment of chronic periodontitis. Periodontol. 2000 2016, 71, 128-139.

20. Sanz, M.; Teughels, W. Innovations in non-surgical periodontal therapy: Consensus 
Report of the Sixth European Workshop on Periodontology. J. Clin. Periodontol. 2008, 35, 3-7.

21. Kapoor, A.; Malhotra, R.; Grover, V.; Grover, D. Systemic antibiotic therapy in periodontics. Dent. Res. J. (Isfahan). 2012, 9, 505.

22. Feres, M.; Figueiredo, L.C.; Soares, G.M.S.; Faveri, M. Systemic antibiotics in the treatment of periodontitis. Periodontol. 2000 2015, 67, 131-186.

23. Winkel, E.G.; Van Winkelhoff, A.J.; Timmerman, M.F.; Van der Velden, U.; Van der Weijden, G.A. Amoxicillin plus metronidazole in the treatment of adult periodontitis patients. A double-blind placebo-controlled study. J. Clin. Periodontol. 2001, 28, 296305.

24. SLOTS, J.; TING, M. Systemic antibiotics in the treatment of periodontal disease. Periodontol. 2000 2002, 28, 106-176.

25. Gajdács, M.; Paulik, E.; Szabó, A. Knowledge, Attitude and Practice of Community Pharmacists Regarding Antibiotic Use and Infectious Diseases: A Cross-Sectional Survey in Hungary (KAPPhA-HU). Antibiotics 2020, 9, 41.

26. Bollen, C.M.L.; Quirynen, M. Microbiological Response to Mechanical Treatment in Combination With Adjunctive Therapy. A Review of the Literature. J. Periodontol. 1996, 67, 1143-1158.

27. Do, M.P.; Neut, C.; Metz, H.; Delcourt, E.; Mäder, K.; Siepmann, J.; Siepmann, F. Insitu forming composite implants for periodontitis treatment: How the formulation determines system performance. Int. J. Pharm. 2015, 486, 38-51.

28. Agarwal, R.K.; Robinson, D.H.; Maze, G.I.; Reinhardt, R.A. Development and characterization of tetracycline-poly(lactide/glycolide) films for the treatment of periodontitis. J. Control. Release 1993, 23, 137-146.

29. Sundararaj, S.C.; Thomas, M. V.; Dziubla, T.D.; Puleo, D.A. Bioerodible system for sequential release of multiple drugs. Acta Biomater. 2014, 10, 115-125.

30. Bromberg, L.E.; Braman, V.M.; Rothstein, D.M.; Spacciapoli, P.; O’Connor, S.M.; Nelson, E.J.; Buxton, D.K.; Tonetti, M.S.; Friden, P.M. Sustained release of silver from periodontal wafers for treatment of periodontitis. J. Control. Release 2000, 68, 63-72.

31. Jain, N.; Jain, G.K.; Javed, S.; Iqbal, Z.; Talegaonkar, S.; Ahmad, F.J.; Khar, R.K. Recent approaches for the treatment of periodontitis. Drug Discov. Today 2008, 13, 932-943.

32. Al-Saeed, M.Y.; Babay, N. The use of povidone-iodine and hydrogen peroxide mixture as an adjunct to non-surgical treatment of slight to moderate chronic periodontitis. Saudi Dent. J. 2009, 21, 127-133.

33. Do, M.P.; Neut, C.; Delcourt, E.; Seixas Certo, T.; Siepmann, J.; Siepmann, F. In situ forming implants for periodontitis treatment with improved adhesive properties. Eur. J. Pharm. Biopharm. 2014, 88, 342-350.

34. Kopytynska-Kasperczyk, A.; Dobrzynski, P.; Pastusiak, M.; Jarzabek, B.; Prochwicz, W. Local delivery system of doxycycline hyclate based on $\epsilon$-caprolactone copolymers for periodontitis treatment. Int. J. Pharm. 2015, 491, 335-344.

35. Chinta, D.P.; Katakam, P.; Murthy, V.S.N.; Newton, M.J. Formulation and in-vitro evaluation of moxifloxacin loaded crosslinked chitosan films for the treatment of periodontitis. J. Pharm. Res. 2013, 7, 483-490.

36. Mayol, L.; Quaglia, F.; Borzacchiello, A.; Ambrosio, L.; Rotonda, M.I. La A novel poloxamers/hyaluronic acid in situ forming hydrogel for drug delivery: Rheological, mucoadhesive and in vitro release properties. Eur. J. Pharm. Biopharm. 2008, 70, 199206.

37. Lee, B.-S.; Lee, C.-C.; Lin, H.-P.; Shih, W.-A.; Hsieh, W.-L.; Lai, C.-H.; Takeuchi, Y.; Chen, Y.-W. A functional chitosan membrane with grafted epigallocatechin-3-gallate and lovastatin enhances periodontal tissue regeneration in dogs. Carbohydr. Polym. 
2016, 151, 790-802.

38. Sundararaj, S.C.; Thomas, M. V.; Peyyala, R.; Dziubla, T.D.; Puleo, D.A. Design of a multiple drug delivery system directed at periodontitis. Biomaterials 2013, 34, 88358842.

39. H.R., R.; Dhamecha, D.; Jagwani, S.; Rao, M.; Jadhav, K.; Shaikh, S.; Puzhankara, L.; Jalalpure, S. Local drug delivery systems in the management of periodontitis: A scientific review. J. Control. Release 2019, 307, 393-409.

40. Kornman, K.S. Controlled-Release Local Delivery Antimicrobials in Periodontics: Prospects for the Future. J. Periodontol. 1993, 64, 782-791.

41. Joshi, D.; Garg, T.; Goyal, A.K.; Rath, G. Advanced drug delivery approaches against periodontitis. Drug Deliv. 2016, 23, 363-377.

42. Tharanathan, R.. Biodegradable films and composite coatings: past, present and future. Trends Food Sci. Technol. 2003, 14, 71-78.

43. Pillai, O.; Panchagnula, R. Polymers in drug delivery. Curr. Opin. Chem. Biol. 2001, 5, 447-451.

44. Langer, R.; Peppas, N.A. Advances in biomaterials, drug delivery, and bionanotechnology. AIChE J. 2003, 49, 2990-3006.

45. Liu, G.; Gu, Z.; Hong, Y.; Cheng, L.; Li, C. Electrospun starch nanofibers: Recent advances, challenges, and strategies for potential pharmaceutical applications. $J$. Control. Release 2017, 252, 95-107.

46. Han, D.; Steckl, A.J. Triaxial Electrospun Nanofiber Membranes for Controlled Dual Release of Functional Molecules. ACS Appl. Mater. Interfaces 2013, 5, 8241-8245.

47. Son, Y.J.; Kim, W.J.; Yoo, H.S. Therapeutic applications of electrospun nanofibers for drug delivery systems. Arch. Pharm. Res. 2014, 37, 69-78.

48. Muzzarelli, R.A.A. Chitins and chitosans for the repair of wounded skin, nerve, cartilage and bone. Carbohydr. Polym. 2009, 76, 167-182.

49. Shelke, S.; Shahi, S.; Jadhav, K.; Dhamecha, D.; Tiwari, R.; Patil, H. Thermoreversible nanoethosomal gel for the intranasal delivery of Eletriptan hydrobromide. J. Mater. Sci. Mater. Med. 2016, 27, 103.

50. Ofluoglu, D.; Ergun, S.; Warnakulasuriya, S.; Namdar-Pekiner, F.; Tanyeri, H. An evaluation of the efficacy of a topical gel with Triester Glycerol Oxide (TGO) in the treatment of minor recurrent aphthous stomatitis in a Turkish cohort: A randomized, double-blind, placebo-controlled clinical trial. Med. Oral Patol. Oral y Cir. Bucal 2017, $0-0$.

51. Lizambard, M.; Menu, T.; Fossart, M.; Bassand, C.; Agossa, K.; Huck, O.; Neut, C.; Siepmann, F. In-situ forming implants for the treatment of periodontal diseases: Simultaneous controlled release of an antiseptic and an anti-inflammatory drug. Int. J. Pharm. 2019, 572, 118833.

52. Bural, C.; Güven, M.; Kayacıoğlu, B.; Ak, G.; Bayraktar, G.; Bilhan, H. Effect of Overthe-Counter Topical Agents on Denture-Induced Traumatic Lesions: A Clinical Study. Int. J. Prosthodont. 2018, 31, 481-484.

53. Zia, K.M.; Tabasum, S.; Khan, M.F.; Akram, N.; Akhter, N.; Noreen, A.; Zuber, M. Recent trends on gellan gum blends with natural and synthetic polymers: A review. Int. J. Biol. Macromol. 2018, 109, 1068-1087.

54. Agarwal, E.; Pradeep, A.R.; Bajaj, P.; Naik, S.B. Efficacy of Local Drug Delivery of $0.5 \%$ Clarithromycin Gel as an Adjunct to Non-Surgical Periodontal Therapy in the Treatment of Current Smokers With Chronic Periodontitis: A Randomized Controlled Clinical Trial. J. Periodontol. 2012, 83, 1155-1163.

55. Jain, M.; Dave, D.; Jain, P.; Manohar, B.; Yadav, B.; Shetty, N. Efficacy of xanthan based chlorhexidine gel as an adjunct to scaling and root planing in treatment of the 
chronic periodontitis. J. Indian Soc. Periodontol. 2013, 17, 439.

56. SHARMA, A.; RAMAN, A.; PRADEEP, A.R. Role of $1 \%$ alendronate gel as adjunct to mechanical therapy in the treatment of chronic periodontitis among smokers. J. Appl. Oral Sci. 2017, 25, 243-249.

57. Gupta, A.; Govila, V.; Pant, V.; Gupta, R.; Verma, U.; Ahmad, H.; Mohan, S. A randomized controlled clinical trial evaluating the efficacy of zoledronate gel as a local drug delivery system in the treatment of chronic periodontitis: A clinical and radiological correlation. Natl. J. Maxillofac. Surg. 2018, 9, 22.

58. Rao, N.; Pradeep, A.; Bajaj, P.; Kumari, M.; Naik, S. Simvastatin local drug delivery in smokers with chronic periodontitis: a randomized controlled clinical trial. Aust. Dent. J. 2013, 58, 156-162.

59. Fehér, A.; Erös, E.C. In situ forming lyotropic liquid crystalline systems containing metronidazole-benzoate. J. Drug Deliv. Sci. Technol. 2005, 15, 343-346.

60. Fehér, A. Formulation and Investigation of in Situ Gelling Lyotropic Liquid Crystalline Systems, University of Szeged, 2006.

61. Arsiwala, A.; Desai, P.; Patravale, V. Recent advances in micro/nanoscale biomedical implants. J. Control. Release 2014, 189, 25-45.

62. Bian, L.; Zhai, D.Y.; Tous, E.; Rai, R.; Mauck, R.L.; Burdick, J.A. Enhanced MSC chondrogenesis following delivery of TGF- $\beta 3$ from alginate microspheres within hyaluronic acid hydrogels in vitro and in vivo. Biomaterials 2011, 32, 6425-6434.

63. Sinha, V..; Singla, A..; Wadhawan, S.; Kaushik, R.; Kumria, R.; Bansal, K.; Dhawan, S. Chitosan microspheres as a potential carrier for drugs. Int. J. Pharm. 2004, 274, 1-33.

64. Yang, Y. Morphology, drug distribution, and in vitro release profiles of biodegradable polymeric microspheres containing protein fabricated by double-emulsion solvent extraction/evaporation method. Biomaterials 2001, 22, 231-241.

65. Tawfik, M.S.; Abdel-Ghaffar, K.A.; Gamal, A.Y.; El-Demerdash, F.H.; Gad, H.A. Lycopene solid lipid microparticles with enhanced effect on gingival crevicular fluid protein carbonyl as a biomarker of oxidative stress in patients with chronic periodontitis. J. Liposome Res. 2019, 29, 375-382.

66. Mundargi, R.C.; Srirangarajan, S.; Agnihotri, S.A.; Patil, S.A.; Ravindra, S.; Setty, S.B.; Aminabhavi, T.M. Development and evaluation of novel biodegradable microspheres based on poly(d,l-lactide-co-glycolide) and poly( $\varepsilon$-caprolactone) for controlled delivery of doxycycline in the treatment of human periodontal pocket: In vitro and in vivo studies. J. Control. Release 2007, 119, 59-68.

67. El-Kamel, A.H.; Ashri, L.Y.; Alsarra, I.A. Micromatricial metronidazole benzoate film as a local mucoadhesive delivery system for treatment of periodontal diseases. AAPS PharmSciTech 2007, 8, E184-E194.

68. Rao, S.K.; Setty, S.; Acharya, A.B.; Thakur, S.L. Efficacy of locally-delivered doxycycline microspheres in chronic localized periodontitis and on Porphyromonas gingivalis. J. Investig. Clin. Dent. 2012, 3, 128-134.

69. Persson, G.R.; Salvi, G.E.; Heitz-Mayfield, L.J.A.; Lang, N.P. Antimicrobial therapy using a local drug delivery system (ArestinR) in the treatment of peri-implantitis. I: microbiological outcomes. Clin. Oral Implants Res. 2006, 17, 386-393.

70. Shin, W.-K.; Cho, J.; Kannan, A.G.; Lee, Y.-S.; Kim, D.-W. Cross-linked Composite Gel Polymer Electrolyte using Mesoporous Methacrylate-Functionalized SiO2 Nanoparticles for Lithium-Ion Polymer Batteries. Sci. Rep. 2016, 6, 26332.

71. Andreu, V.; Arruebo, M. Current progress and challenges of nanoparticle-based therapeutics in pain management. J. Control. Release 2018, 269, 189-213.

72. Zazo, H.; Colino, C.I.; Lanao, J.M. Current applications of nanoparticles in infectious diseases. J. Control. Release 2016, 224, 86-102. 
73. Jadhav, K.; Dhamecha, D.; Bhattacharya, D.; Patil, M. Green and ecofriendly synthesis of silver nanoparticles: Characterization, biocompatibility studies and gel formulation for treatment of infections in burns. J. Photochem. Photobiol. B Biol. 2016, 155, 109115.

74. Cioffi, N.; Torsi, L.; Ditaranto, N.; Tantillo, G.; Ghibelli, L.; Sabbatini, L.; BleveZacheo, T.; D’Alessio, M.; Zambonin, P.G.; Traversa, E. Copper Nanoparticle/Polymer Composites with Antifungal and Bacteriostatic Properties. Chem. Mater. 2005, 17, 5255-5262.

75. Jadhav, K.; Deore, S.; Dhamecha, D.; H R, R.; Jagwani, S.; Jalalpure, S.; Bohara, R. Phytosynthesis of Silver Nanoparticles: Characterization, Biocompatibility Studies, and Anticancer Activity. ACS Biomater. Sci. Eng. 2018, 4, 892-899.

76. Vial, S.; Reis, R.L.; Oliveira, J.M. Recent advances using gold nanoparticles as a promising multimodal tool for tissue engineering and regenerative medicine. Curr. Opin. Solid State Mater. Sci. 2017, 21, 92-112.

77. Kong, L.X.; Peng, Z.; Li, S.-D.; Bartold, P.M. Nanotechnology and its role in the management of periodontal diseases. Periodontol. 2000 2006, 40, 184-196.

78. Atridox FDA label Available online: https://www.accessdata.fda.gov/drugsatfda_docs/label/2011/050751s015lbl.pdf (accessed on Feb 9, 2020).

79. Actisite - FDA Available online: https://www.accessdata.fda.gov/scripts/cder/daf/index.cfm?event=overview.process\&A pplNo=050653 (accessed on Feb 9, 2020).

80. Actisite - RxList Available online: https://www.rxlist.com/actisite-drug.htm\#description (accessed on Feb 9, 2020).

81. PerioChip FDA label Available online: https://www.accessdata.fda.gov/drugsatfda_docs/label/2012/020774s011lbl.pdf (accessed on Feb 9, 2020).

82. ARESTIN FDA label Available online: https://www.accessdata.fda.gov/drugsatfda_docs/label/2017/050781s020lbl.pdf (accessed on Feb 9, 2020).

83. Jannin, V.; Musakhanian, J.; Marchaud, D. Approaches for the development of solid and semi-solid lipid-based formulations. Adv. Drug Deliv. Rev. 2008, 60, 734-746.

84. Pouton, C.W. Lipid formulations for oral administration of drugs: non-emulsifying, selfemulsifying and "self-microemulsifying" drug delivery systems. Eur. J. Pharm. Sci. 2000, 11 Suppl 2, S93-8.

85. Pouton, C.W.; Porter, C.J.H. Formulation of lipid-based delivery systems for oral administration: materials, methods and strategies. Adv. Drug Deliv. Rev. 2008, 60, 62537.

86. Wang, L.; Ryan, A.J. Introduction to electrospinning. Electrospinning Tissue Regen. 2011, 3-33.

87. Huang, Z.M.; Zhang, Y.Z.; Kotaki, M.; Ramakrishna, S. A review on polymer nanofibers by electrospinning and their applications in nanocomposites. Compos. Sci. Technol. 2003, 63, 2223-2253.

88. Zheng, F.; Wang, S.; Wen, S.; Shen, M.; Zhu, M.; Shi, X. Characterization and antibacterial activity of amoxicillin-loaded electrospun nano-hydroxyapatite/poly(lacticco-glycolic acid) composite nanofibers. Biomaterials 2013, 34, 1402-1412.

89. Ranjbar-Mohammadi, M.; Zamani, M.; Prabhakaran, M.P.; Bahrami, S.H.; Ramakrishna, S. Electrospinning of PLGA/gum tragacanth nanofibers containing tetracycline hydrochloride for periodontal regeneration. Mater. Sci. Eng. C 2016, 58, $521-531$. 
90. Khan, G.; Yadav, S.K.; Patel, R.R.; Kumar, N.; Bansal, M.; Mishra, B. Tinidazole functionalized homogeneous electrospun chitosan/poly ( $\varepsilon$-caprolactone) hybrid nanofiber membrane: Development, optimization and its clinical implications. Int. J. Biol. Macromol. 2017, 103, 1311-1326.

91. Schkarpetkin, D.; Reise, M.; Wyrwa, R.; Völpel, A.; Berg, A.; Schweder, M.; Schnabelrauch, M.; Watts, D.C.; Sigusch, B.W. Development of novel electrospun dualdrug fiber mats loaded with a combination of ampicillin and metronidazole. Dent. Mater. 2016, 32, 951-960.

92. Dias, A.M.; da Silva, F.G.; Monteiro, A.P. de F.; Pinzón-García, A.D.; Sinisterra, R.D.; Cortés, M.E. Polycaprolactone nanofibers loaded oxytetracycline hydrochloride and zinc oxide for treatment of periodontal disease. Mater. Sci. Eng. C 2019, 103, 109798.

93. Sun, B.; Long, Y.Z.; Zhang, H.D.; Li, M.M.; Duvail, J.L.; Jiang, X.Y.; Yin, H.L. Advances in three-dimensional nanofibrous macrostructures via electrospinning. Prog. Polym. Sci. 2014, 39, 862-890.

94. Wang, N.; Zhao, Y. Coaxial Electrospinning. In Electrospinning: Nanofabrication and Applications; Elsevier, 2019; pp. 125-200.

95. He, J.; Zhou, Y. Multineedle Electrospinning. In Electrospinning: Nanofabrication and Applications; Elsevier, 2019; pp. 201-218.

96. Yan, G.; Niu, H.; Lin, T. Needle-less Electrospinning. In Electrospinning: Nanofabrication and Applications; Elsevier, 2019; pp. 219-247.

97. Zhang, S.; Rind, N.A.; Tang, N.; Liu, H.; Yin, X.; Yu, J.; Ding, B. Electrospun Nanofibers for Air Filtration. In Electrospinning: Nanofabrication and Applications; Elsevier, 2019; pp. 365-389.

98. Sarbatly, R.; Krishnaiah, D.; Kamin, Z. A review of polymer nanofibres by electrospinning and their application in oil-water separation for cleaning up marine oil spills. Mar. Pollut. Bull. 2016, 106, 8-16.

99. Wasim, M.; Sabir, A.; Shafiq, M.; Jamil, T. Electrospinning: A Fiber Fabrication Technique for Water Purification. In Nanoscale Materials in Water Purification; Elsevier, 2019; pp. 289-308.

100. Ding, B.; Wang, M.; Yu, J.; Sun, G. Gas Sensors Based on Electrospun Nanofibers. Sensors 2009, 9, 1609-1624.

101. Sill, T.J.; von Recum, H.A. Electrospinning: Applications in drug delivery and tissue engineering. Biomaterials 2008, 29, 1989-2006.

102. Torres-Martinez, E.J.; Cornejo Bravo, J.M.; Serrano Medina, A.; Pérez González, G.L.; Villarreal Gómez, L.J. A Summary of Electrospun Nanofibers as Drug Delivery System: Drugs Loaded and Biopolymers Used as Matrices. Curr. Drug Deliv. 2018, 15, 13601374.

103. Hu, X.; Liu, S.; Zhou, G.; Huang, Y.; Xie, Z.; Jing, X. Electrospinning of polymeric nanofibers for drug delivery applications. J. Control. Release 2014, 185, 12-21.

104. Sutherland, R.; Croydon, E.A.P.; Rolinson, G.N. Amoxycillin: A new Semi-synthetic Penicillin. Br. Med. J. 1972, 3, 13-16.

105. Edwards, D.I. Mechanisms of selective toxicity of metronidazole and other nitroimidazole drugs. Br. J. Vener. Dis. 1980, 56, 285-290.

106. Juhász, I.; Zoltán, P.; Erdei, I. Treatment of partial thickness burns with Zn-hyaluronan: lessons of a clinical pilot study. Ann. Burns Fire Disasters 2012, 25, 82-5.

107. Horvát, G.; Budai-Szucs, M.; Berkó, S.; Szabó-Révész, P.; Soós, J.; Facskó, A.; Maroda, M.; Mori, M.; Sandri, G.; Bonferoni, M.C.; et al. Comparative study of nanosized crosslinked sodium-, linear sodium- and zinc-hyaluronate as potential ocular mucoadhesive drug delivery systems. Int. J. Pharm. 2015, 494, 321-328.

108. Dahiya, P.; Kamal, R. Hyaluronic acid: A boon in periodontal therapy. N. Am. J. Med. 
Sci. 2013, 5, 309.

109. Leber, A.; Budai-Szucs, M.; Urban, E.; Valyi, P.; Kovacs, A.; Berko, S.; Csanyi, E. Formulation and Investigation of a Lipid Based Delivery System Containing Antimicrobials for the Treatment of Periodontal Disease. Curr. Drug Deliv. 2018, 15.

110. Perioli, L.; Ambrogi, V.; Angelici, F.; Ricci, M.; Giovagnoli, S.; Capuccella, M.; Rossi, C. Development of mucoadhesive patches for buccal administration of ibuprofen. $J$. Control. Release 2004, 99, 73-82.

111. Ramesha Chary, R.B.; Vani, G.; Rao, Y.M. In Vitro and In Vivo Adhesion Testing of Mucoadhesive Drug Delivery Systems. Drug Dev. Ind. Pharm. 1999, 25, 685-690.

112. C. Rowe, R.; J. Sheskey, P.; E. Quinn, M. Handbook of Pharmaceutical Excipients; 6th editio.; Pharmaceutical Press, 2009; ISBN 9780853697923.

113. P. Pawar, R.; U. Tekale, S.; U. Shisodia, S.; T. Totre, J.; J. Domb, A. Biomedical Applications of Poly(Lactic Acid). Rec. Pat. Regen. Med. 2014, 4, 40-51.

114. Léber, A.; Budai-Szücs, M.; Urbán, E.; Vályi, P.; Gácsi, A.; Berkó, S.; Kovács, A.; Csányi, E. Combination of Zinc Hyaluronate and Metronidazole in a Lipid-Based Drug Delivery System for the Treatment of Periodontitis. Pharmaceutics 2019, 11, 142.

115. Chen, C.K.C.; Wilson, M.E. Eikenella corrodens in Human Oral and Non-Oral Infections: A Review. J. Periodontol. 1992, 63, 941-953.

116. Baghban, A.; Gupta, S. Parvimonas micra: A rare cause of native joint septic arthritis. Anaerobe 2016, 39, 26-27.

117. Rams, T.E.; Feik, D.; Listgarten, M.A.; Slots, J. Peptostreptococcus micros in human periodontitis. Oral Microbiol. Immunol. 1992, 7, 1-6.

118. Santos, F.A.; Bastos, E.M.A.; Rodrigues, P.H.; de Uzeda, M.; de Carvalho, M.A.R.; de Macedo Farias, L.; Andrade Moreira, E.S. Susceptibility of Prevotella intermedia/Prevotella nigrescens (and Porphyromonas gingivalis) to Propolis (Bee Glue) and other Antimicrobial Agents. Anaerobe 2002, 8, 9-15.

119. Han, Y.W. Fusobacterium nucleatum: a commensal-turned pathogen. Curr. Opin. Microbiol. 2015, 23, 141-147.

120. Jacinto, R.C.; Montagner, F.; Signoretti, F.G.C.; Almeida, G.C.; Gomes, B.P.F.A. Frequency, Microbial Interactions, and Antimicrobial Susceptibility of Fusobacterium nucleatum and Fusobacterium necrophorum Isolated from Primary Endodontic Infections. J. Endod. 2008, 34, 1451-1456.

121. Raja, M. Aggregatibacter Actinomycetemcomitans - A Tooth Killer? J. Clin. DIAGNOSTIC Res. 2014, 8, 13-16.

122. Oettinger-Barak, O.; Dashper, S.G.; Catmull, D. V.; Adams, G.G.; Sela, M.N.; Machtei, E.E.; Reynolds, E.C. Antibiotic susceptibility of Aggregatibacter actinomycetemcomitans JP2 in a biofilm. J. Oral Microbiol. 2013, 5, 20320.

123. How, K.Y.; Song, K.P.; Chan, K.G. Porphyromonas gingivalis: An Overview of Periodontopathic Pathogen below the Gum Line. Front. Microbiol. 2016, 7, 1-14.

124. Jacinto, R.C.; Gomes, B.P.F.A.; Shah, H.N.; Ferraz, C.C.; Zaia, A.A.; Souza-Filho, F.J. Incidence and antimicrobial susceptibility of Porphyromonas gingivalis isolated from mixed endodontic infections. Int. Endod. J. 2006, 39, 62-70.

125. József, B.; István, V.; Kinga, B.; István, L.; Viktória, H.; Igor, B.; Csaba, H. PerioChip ${ }^{\circledR}$ klórhexidin-glükonát felszabadulásának vizsgálata különböző pH-k esetén. Fogorv. Sz. 2019, 112., 34-40.

126. Moore, W.E.C.; Moore, L.V.H. The bacteria of periodontal diseases. Periodontol. 2000 1994, 5, 66-77.

127. Costalonga, M.; Herzberg, M.C. The oral microbiome and the immunobiology of periodontal disease and caries. Immunol. Lett. 2014, 162, 22-38.

128. Kolenbrander, P.E.; Palmer, R.J.; Periasamy, S.; Jakubovics, N.S. Oral multispecies 
biofilm development and the key role of cell-cell distance. Nat. Rev. Microbiol. 2010, 8, 471-480.

129. Mani, A.; Pawar, B.; Pendyala, G.; Mustilwar, R.; Bhosale, A.; Bhadange, S. Hyaluronic acid - A boon to periodontal therapy. Pravara Med. Rev. 2016, 8, 8-13.

130. Adams, D.; Addy, M. Mouthrinses. Adv. Dent. Res. 1994, 8, 291-301.

131. Goudouri, O.; Kontonasaki, E.; Lohbauer, U.; Boccaccini, A.R. Antibacterial properties of metal and metalloid ions in chronic periodontitis and peri-implantitis therapy. Acta Biomater. 2014, 10, 3795-3810.

132. Sutherland, R.; Croydon, E. a; Rolinson, G.N. Amoxycillin: a new semi-synthetic penicillin. Br. Med. J. 1972, 3, 13-16.

133. Li, Z.Q.; Zhou, X.D.; Pei, C.H. Synthesis of PLA-co-PGMA Copolymer and its Application in the Surface Modification of Bacterial Cellulose. Int. J. Polym. Mater. 2010, 59, 725-737.

134. Jordá-Vilaplana, A.; Fombuena, V.; García-García, D.; Samper, M.D.; Sánchez-Nácher, L. Surface modification of polylactic acid (PLA) by air atmospheric plasma treatment. Eur. Polym. J. 2014, 58, 23-33.

135. Biresaw, G.; Carriere, C.J. Interfacial tension of poly(lactic acid)/polystyrene blends. $J$. Polym. Sci. Part B Polym. Phys. 2002, 40, 2248-2258.

136. Kenyó, C.; Kajtár, D.A.; Renner, K.; Kröhnke, C.; Pukánszky, B. Functional packaging materials: factors affecting the capacity and rate of water adsorption in desiccant composites. J. Polym. Res. 2013, 20, 294.

137. Zhang, J.J.; Liu, J.; Yu, H.; Zhang, Y.; Zhu, M.F.; Chen, Y.M. Crosslinked Electrospun UPM/PHBV/PVP Fibers for Sustained Drug Release. Mater. Sci. Forum 2009, 610-613, 1331-1334.

138. Yohe, S.T.; Colson, Y.L.; Grinstaff, M.W. Superhydrophobic Materials for Tunable Drug Release: Using Displacement of Air To Control Delivery Rates. J. Am. Chem. Soc. 2012, 134, 2016-2019. 


\section{ACKNOWLEDGEMENTS}

I would like to express my sincere gratitude to the Head of the Institute of Pharmaceutical Technology and Regulatory Affairs Dr. habil. Ildikó Csóka for providing me with the opportunity to work in this Department and complete my Ph.D. work under her expert guidance.

I would like to express my warmest thanks to my supervisors Dr. habil. Erzsébet Csányi associate professor and Dr. Mária Budai-Szúcs assistant professor for their guidance, patience, motivation and continuous support of my research.

I wish to thank Dr. Attila Gácsi for his immense help and co-operation, especially in HPLC measurements.

I wish to thank Dr. habil. Edit Urbán and Dr. habil. Katalin Burián the former and current head of the Institute of Clinical Microbiology, University of Szeged for their immense help with the antimicrobial investigations.

I owe many thanks to Dr. Péter Vályi for his professional guidance on periodontal diseases.

I am very grateful to all my co-authors, Prof. Dr. Béla Pukánszky, Dr. Balázs Kirschweng, Muriel Józó and Lu Cui from the Laboratory of Plastics and Rubber Technology, Budapest University of Technology and Economics, for their co-operation.

I wish to thank all members of the Institute of Pharmaceutical Technology and Regulatory Affairs for their help and friendship.

I also owe many thanks to my family and friends for their encouragement, support and understanding; and for ensuring a peaceful background. 
ANNEX 
I. 
RESEARCH ARTICLE

\title{
Formulation and Investigation of a Lipid Based Delivery System Contain- ing Antimicrobials for the Treatment of Periodontal Disease
}

\author{
Attila Léber ${ }^{\mathrm{a}}$, Mária Budai-Szűcs ${ }^{\mathrm{a}}$, Edit Urbán ${ }^{\mathrm{b}}$, Péter Vályi ${ }^{\mathrm{c}}$, Anita Kovács ${ }^{\mathrm{a}}$, Szilvia Berkóa and \\ Erzsébet Csányi $*^{\mathrm{a}}$ \\ ${ }^{a}$ Institute of Pharmaceutical Technology and Regulatory Affairs, Faculty of Pharmacy, University of Szeged; ${ }^{b}$ Institute \\ of Clinical Microbiology, Faculty of Medicine, University of Szeged; ${ }^{c}$ Department of Periodontology, Faculty of Den- \\ tistry, University of Szeged
}

\begin{abstract}
Background: Periodontitis is a chronic inflammatory disease, which affects the supporting tissues of the teeth, and without proper treatment it may lead to tooth loss. Antibiotics - administered orally - have been widely used in the treatment of periodontitis. With the conventional administration routes, adequate drug levels cannot be reached in the periodontal pockets and oral application of antimicrobials could lead to side effects. Drug delivery systems containing antibiotics, administered at the site of infection, could possibly help eliminate pathogen bacteria and treat periodontitis.
\end{abstract}

\section{A R T I C L E H IS T O R Y \\ Received: August 24, 2017 Revised: December 11,2017 Revised: Decepted: Janury 10,2018 \\ DOI: 10.2174/1567201815666180116092354}

Objective: The aim of the recent study was to create a locally swellable, biodegradable, biocompatible, mucoadhesive, lipophilic drug delivery system (SDLDDS) containing antimicrobial drugs which softens at body temperature, accommodate to the shape of the periodontal pocket and can provide extended drug release for at least one week.

Methods: During the formulation, thermoanalytical, consistency, wettability, swelling, degradation and drug release studies were applied to determine the ideal ratios of lipid bases, structure-building components and surface active agent concentrations.

Results and Discussion: The structure-building component cetostearyl alcohol appeared to be the most convenient, thanks to its wettability and mechanical properties, which led to controlled drug release. With the use of ideal concentrations of components (10\% surfactant, $40 \%$ structure-building component, $32 \%$ lipid base, $15 \%$ antimicrobial agent and $3 \%$ polymer), sustained drug release can be provided up to nearly 3 weeks.

Keywords: lipid drug delivery, sustained drug release, swelling, degradation, periodontitis, periodontal pocket, antimicrobial therapy.

\section{INTRODUCTION}

Periodontitis (PD) is a chronic inflammatory disease, which affects the supporting tissues of the teeth, and without proper treatment it may lead to tooth loss $[1,2]$. Periodontal diseases are multifactorial diseases: initiation and progression of periodontitis require the simultaneous occurrence of virulent periodontal pathogen microorganisms, local environment, host susceptibility, genetic and acquired risk factors. The subgingival biofilm initiates and maintains inflammatory responses in the periodontal tissues and periodontopathogen microorganisms also play a role directly in tissue damage through different virulence factors [3-5]. Inflammation is mostly caused by a wide set of microorganisms

*Address correspondence to this author at the Institute of Pharmaceutical Technology and Regulatory Affairs, Faculty of Pharmacy, University of Szeged, Eötvös st. 6., Szeged, Hungary; Tel: +36-62-545-573; Fax: +36-62545-571; E-mail: csanyi@pharm.u-szeged.hu (viruses, bacteria, etc.), with the most important putative pathogens being Gram-negative bacteria, namely Aggregatibacter actinomycetemcomitans, Porphyromonas gingivalis, Tannerella forsythia and Treponema denticola, but other species are also considered to be responsible for disease initiation and progression [6].

The disease is characterised by the destruction of the gingival tissues, the resorption of the alveolar bone, the migration of the junctional epithelium in the direction of the roots and the formation of a so-called periodontal pocket (PP) [2] This subgingival area is ideal for the proliferation of anaerobic pathogen bacteria, some of which were listed above, which activate neutral degrading enzymes, thereby causing a vicious cycle $[2,6]$.

The oral cavity and PPs have all the important features required to be an appropriate medium for the growth and maturation of a bacterial biofilm [6]. A community of bacte- 
rial cells producing a polymeric matrix in which they are enclosed on a surface is called a bacterial biofilm [7]. As there are lipids, saccharides in the nutrients and proteins and glycoproteins in saliva, bacteria have access to adequate nourishment [6].

As biofilm formation and adherence to tooth surface are the main causes of periodontal disease, the removal of plaque and the eradication of microorganisms are key elements of an effective therapy [8-10]. The most common methods of mechanical debridement are scaling and root planing with some limitations, such as the inability to access ecological niches. The varying geometry of deep pockets, the irregularities of the root surface, root furcations, concavities and infected pocket walls are not accessible to mechanical removal by hand or with power-driven instruments [11]. Because of the imperfect removal of the bacterial biofilm and the high number of pathogen microorganisms present in the oral cavity, chances are high that the remaining microbiota recolonises the subgingival area [12].

The infectious nature of periodontitis indicates an adjunctive anti-infective chemotherapy to eradicate or reduce the amounts of pathogenic bacteria from ecological niches. Our current understanding of antimicrobial therapy requires the disruption of the structured biofilm by mechanical debridement before antimicrobial therapy is considered [11].

Antibiotics have been widely used in the treatment of PD and can help eliminate the oral pathogens responsible for the formation of the disease. However, with conventional administration routes (e.g. per os), adequate drug levels are not reachable in the PPs even with high systemic concentrations. The oral application of high doses of antimicrobial agents leads to serious side effects with limited efficacy [13].

As the peroral application of antimicrobial drugs is not a viable option, antimicrobial local drug delivery systems applied at the inflammation site could be a possibility in the elimination of pathogen bacteria and in the treatment of PD. Numerous articles demonstrated various types of local drug delivery systems with different active agents directed at PD [1, 2, 12-25]. Applicable active substances could be chlorhexidine, metronidazole, tetracyclines, clindamycin, ofloxacin [2], moxifloxacin [17], amoxicillin [18] or even povidone iodine [26].

The development of local, targeted, sustained and controlled release therapeutic systems containing different active agents are among the hot topics of pharmaceutical research. Serious adverse effects of particular drugs can be prevented by their administration, and even a continuous effect could be obtained without sequential dosage and therapeutic drug levels can be achieved at the desired place. Different nano carriers [27], layered structures [22, 28], polymer matrixes $[27,29]$ and lipid systems $[30,31]$ may be used to achieve these features.

Sustained or controlled release of drugs can be also obtained with the use of different vehicles like polymers or other materials capable of up-building a coherent structure [28, 32-34].

The aim of the recent study was to create a locally swellable, degradable, lipophilic drug delivery system (SDLDDS) containing an antimicrobial drug, which softens at body temperature, accommodates to the shape of the PP, is biodegradable, biocompatible, can provide extended drug release at least for one week and has a mucoadhesive feature.

\section{MATERIALS AND METHODS}

\subsection{Materials}

For the compositions Suppocire BP (SBP) pellets (Gattefossé, Saint-Priest, France), Methocel E4M (Colorcon Ltd., Dartford, United Kingdom), Kolliphor RH40 (KP) (BASF ChemTrade GmbH, Ludwigshafen, Germany), amoxicillin (AMX) (Antibióticos de León S.L.U., León, Spain), white beeswax (WB), cetostearyl alcohol (CA) and metronidazole (MZ) (Ph. Eur. 8., Hungaropharma Plc., Budapest, Hungary) were used.

During the drug release tests, a simulated saliva solution was used as an acceptor phase. The composition of the solution was the following: $0.720 \mathrm{~g} / \mathrm{L}$ potassium chloride, 0.600 $\mathrm{g} / \mathrm{L}$ sodium chloride, $0.030 \mathrm{~g} / \mathrm{L}$ citric acid (Ph. Eur. 8., Hungaropharma Plc., Budapest, Hungary), $0.220 \mathrm{~g} / \mathrm{L}$ calcium chloride dihydrate, $0.680 \mathrm{~g} / \mathrm{L}$ potassium phosphate monobasic, $0.866 \mathrm{~g} / \mathrm{L}$ sodium phosphate dodecahydrate, dibasic, $1.500 \mathrm{~g} / \mathrm{L}$ potassium bicarbonate and $0.060 \mathrm{~g} / \mathrm{L}$ potassium thiocyanate (Ph. Eur. 8., Molar Chemicals Ltd., Halásztelek, Hungary) at a pH of 6.5 [35]. For wettability, swelling and degradation measurements purified water was used.

The preliminary microbiological investigations were implemented with the use of a test bacterium, Streptococcus mutans (ATCC ${ }^{\circledR} 55677^{\mathrm{TM}}$ ), a facultatively anaerobic, oral streptococcus. Bacterial suspensions were made in $0.9 \%$ $\mathrm{NaCl}$ solution. Columbia agar plates with $5 \%$ of horse blood were used as culture media.

\subsection{Composition of the Local Drug Delivery Systems}

Five main components were used to create the delivery systems. A lipophilic component SBP was chosen as the lipid base and CA or WB was used as a structure-building component. All of the lipid components can be safely used on mucosae, where the SBP can soften at body temperature. Each structure-building component was used in four different concentrations, from which the best was chosen according to the results of different measurements.

KP was used as a surface active agent, which helps the wetting of the formulation in the PP and thus, promotes its swelling and degradation. Different concentrations were applied to decide which is the most suitable for the delivery system.

Methocel E4M (HPMC) was used as a swelling and bioadhesive agent.

AMX or MZ, which are often used in PD treatment, were incorporated into the vehicles.

All of the formulations were created by melting. At first, the two lipophilic components and the surfactant were melted together at $70{ }^{\circ} \mathrm{C}$ on a hot plate (IKA-WERKE RCT B, ETSD4, IKA Werke GmbH \& Co. KG, Staufen im Breisgau, Germany), then the polymer was added and homogenized with an overhead stirrer at 50 RPM (Digital Overhead Stirrer DLH, VELP Scientifica, Usmate Velate, Italy). The antimicrobial agent was only added right before moulding to avoid 
its possible thermal decomposition. Delivery systems were created by moulding the melted formulations into a round shaped mould $1.5 \mathrm{~mm}$ in thickness and $9 \mathrm{~mm}$ in diameter.

The optimal ratio of the ingredients was determined by various methods described below. Before any measurement, the formulations were kept at room temperature in a desiccator for at least $24 \mathrm{~h}$ to reach complete solidification.

The compositions of different formulations examined during our study can be found in Table $\mathbf{1}$. The course of the investigations is shown in Fig. (1).

\subsection{Differential Scanning Calorimetry (DSC) Measure- ments}

DSC measurements were used to determine the softening temperature and the effect of the structure-building component on the melting point and the structure. All of the measurements were done with a Mettler-Toledo DSC $821^{\mathrm{e}}$ instrument in argon atmosphere $(100 \mathrm{~mL} / \mathrm{min})$ between +10 and $100{ }^{\circ} \mathrm{C}$ raising the temperature by $5{ }^{\circ} \mathrm{C}$ per minute. 10 $\mathrm{mg}$ of the samples were placed into $40-\mu \mathrm{L}$ aluminium pans. The pans were sealed and holed on top.

Table 1. The composition of formulations analysed with different methods.

\begin{tabular}{|c|c|c|c|c|c|c|c|}
\hline \multirow{3}{*}{ Measurements } & \multicolumn{7}{|c|}{ Concentration of the Components (\%) } \\
\hline & \multicolumn{2}{|c|}{$\begin{array}{l}\text { Structure-building } \\
\text { Component }\end{array}$} & \multirow{2}{*}{$\begin{array}{c}\text { Lipid Base } \\
\text { SBP }\end{array}$} & \multirow{2}{*}{$\begin{array}{c}\text { Surfactant } \\
\text { KP }\end{array}$} & \multirow{2}{*}{$\begin{array}{c}\text { Polymer } \\
\text { Methocel E4M } \\
\end{array}$} & \multicolumn{2}{|c|}{ Antimicrobials } \\
\hline & WB & CA & & & & $\mathbf{M Z}$ & AMX \\
\hline \multirow{14}{*}{$\begin{array}{c}\text { DSC, } \\
\text { Wettability, } \\
\text { Penetrometry }\end{array}$} & 10 & 0 & 85 & 5 & 0 & 0 & 0 \\
\hline & 10 & 0 & 80 & 10 & 0 & 0 & 0 \\
\hline & 10 & 0 & 75 & 15 & 0 & 0 & 0 \\
\hline & 10 & 0 & 70 & 20 & 0 & 0 & 0 \\
\hline & 0 & 5 & 90 & 5 & 0 & 0 & 0 \\
\hline & 0 & 5 & 85 & 10 & 0 & 0 & 0 \\
\hline & 0 & 5 & 80 & 15 & 0 & 0 & 0 \\
\hline & 0 & 5 & 75 & 20 & 0 & 0 & 0 \\
\hline & 20 & 0 & 67 & 10 & 3 & 0 & 0 \\
\hline & 40 & 0 & 47 & 10 & 3 & 0 & 0 \\
\hline & 60 & 0 & 27 & 10 & 3 & 0 & 0 \\
\hline & 0 & 20 & 67 & 10 & 3 & 0 & 0 \\
\hline & 0 & 40 & 47 & 10 & 3 & 0 & 0 \\
\hline & 0 & 60 & 27 & 10 & 3 & 0 & 0 \\
\hline \multirow{6}{*}{ Swelling and degradation } & 20 & 0 & 67 & 10 & 3 & 0 & 0 \\
\hline & 40 & 0 & 47 & 10 & 3 & 0 & 0 \\
\hline & 60 & 0 & 27 & 10 & 3 & 0 & 0 \\
\hline & 0 & 20 & 67 & 10 & 3 & 0 & 0 \\
\hline & 0 & 40 & 47 & 10 & 3 & 0 & 0 \\
\hline & 0 & 60 & 27 & 10 & 3 & 0 & 0 \\
\hline \multirow{9}{*}{ Drug release study } & 20 & 0 & 57 & 10 & 3 & 10 & 0 \\
\hline & 40 & 0 & 37 & 10 & 3 & 10 & 0 \\
\hline & 60 & 0 & 17 & 10 & 3 & 10 & 0 \\
\hline & 0 & 20 & 57 & 10 & 3 & 10 & 0 \\
\hline & 0 & 40 & 37 & 10 & 3 & 10 & 0 \\
\hline & 0 & 60 & 17 & 10 & 3 & 10 & 0 \\
\hline & 0 & 20 & 57 & 10 & 3 & 0 & 10 \\
\hline & 0 & 40 & 37 & 10 & 3 & 0 & 10 \\
\hline & 0 & 60 & 17 & 10 & 3 & 0 & 10 \\
\hline \multirow{3}{*}{$\begin{array}{l}\text { Preliminary microbiological } \\
\text { study }\end{array}$} & 0 & 40 & 42 & 10 & 3 & 0 & 5 \\
\hline & 0 & 40 & 37 & 10 & 3 & 0 & 10 \\
\hline & 0 & 40 & 32 & 10 & 3 & 0 & 15 \\
\hline
\end{tabular}


Determination of the optimal concentration ratios

Selection of the possible main components

Determination of the test sample compositions

DSC, wettability and consistency measurements

$\sqrt{1}$

Determination of the concentration of the surfaceactive agent and the structure-building component

Swelling, degradation and drug diffusion tests

Determination of the most convenient structurebuilding component and its optimal concentration

Preliminary microbiological investigation

Fig. (1). Flowchart showing the course of the investigations.

\subsection{Wettability of the Delivery Systems}

The wettability of the formulations was studied with an OCA Contact Angle System (Dataphysics OCA 20, Dataphysics Inc., GmbH, Germany). As a flat surface is needed for optical contact angle measurements to be carried out, microscopic slides were covered with the melted formulations (without active ingredients). After moulding, they were stored at room temperature in a desiccator for $24 \mathrm{~h}$. The degree of wetting was determined by measuring the contact angle: $10 \mu \mathrm{L}$ of water from a calibrated syringe was dripped on the surface of the formulations moulded onto the microscopic slides, and the contact angle of the water droplets and the formulations was measured by computer software.

\subsection{Determination of Consistency by Penetrometry}

The consistency of the different formulations was determined with a penetrometer (07/2008:20909, 2.9.9, 2.9.9.-1,3., Ph. Eur. 8) by measuring the penetration depth of a penetrating object into the test samples. Melted samples were poured into Petri dishes, and they were stored at room temperature for $24 \mathrm{~h}$ in a desiccator to reach the total solidification. Five parallel measurements were carried out with each sample at room temperature. The penetration depth was measured for one minute. The penetration depth directly refers to the consistency of the samples.

\subsection{Swelling and Degradation}

The water absorption capacity of the formulations was determined gravimetrically in order to examine the effects of the incorporated polymer on the swelling and degradation properties of the delivery systems. At first, the formulations were weighed with an analytical scale then were immersed into $10 \mathrm{~mL}$ of distilled water and were thermostated at $37^{\circ} \mathrm{C}$ for various periods of time $(1,1.5,2,3,4,5,6,7$ and 14 days). After the defined time periods, they were weighed again. After the second measurement, the formulations were held at room temperature until they totally dried out, and they were weighed again for a third time. With the results, the absorption capacity and the swelling related degradation could be determined.

\subsection{Drug Release Study}

The in vitro drug release profiles of the formulations were determined. The compositions were weighed with an analytical scale and put in 5-cm-long dialysis tubes (Spectra/Por ${ }^{\circledR}$ Standard RC tubing, MWCO: $12-14 \mathrm{kD}$ ) and sealed with closures. The tubes containing the formulations were placed in $50 \mathrm{~mL}$ of acceptor phase thermostated at $37{ }^{\circ} \mathrm{C}$. As an acceptor phase, a simulated saliva solution was used. Drug release was investigated for $48 \mathrm{~h}$ and 3 parallel measurements were carried out. $5 \mathrm{~mL}$ of samples were taken (at $0.5,1,4,8,12,24,36,48$ hours) and were replaced with 5 $\mathrm{mL}$ of acceptor phase. The released drug, AMX and MZ, was quantified by UV spectrophotometry (Helios $\alpha$ Thermospectronic UV-spectrophotometer v4.55, Unicam: Thermo Fisher Scientific, Waltham, MA) at 273 and $318 \mathrm{~nm}$, respectively.

\subsection{Investigation of Sustained Antimicrobial Effect: Pre- liminary Study}

A preliminary microbiological study was conducted in order to measure the antibacterial effectiveness of the developed formulations. As a typical oral bacterium, Streptococcus mutans control strain was used. A $0.5 \mathrm{McF}$ arland standard concentration bacterial suspension was made with $0.9 \%$ $\mathrm{NaCl}$ solution (in suspension it is equivalent to approximately $1.5 \times 10^{8}$ colony forming units $/ \mathrm{mL}$ ). The suspension was spread onto a horse blood agar plate, then formulations containing 5, 10 and $15 \mathrm{~m} / \mathrm{m} \%$ of AMX were placed on it. After 24 hours of incubation in $5 \% \mathrm{CO}_{2}$ atmosphere, the diameter of the inhibition zones was measured. The formulations were then put on a new horse blood agar plate, also inoculated with 0.5 McFarland standard concentration freshly made bacterial suspension of Streptococcus mutans. The plates were then put in $5 \% \mathrm{CO}_{2}$ atmosphere for 24 hours. This was repeated until no inhibition zone could be detected.

\subsection{Statistical Analysis}

The results of the drug release measurements were analysed statistically with GraphPad Prism version 5 software, using two-way ANOVA analysis with Bonferroni post-tests. A level of $p \leq 0.05$ was considered significant, $p \leq 0.01$, very significant, and $\mathrm{p} \leq 0.001$ highly significant.

\section{RESULTS AND DISCUSSION}

\subsection{Differential Scanning Calorimetry (DSC) Measure- ments}

DSC was used to determine the concentration of the structure-building component and the softening temperature of the formulations.

One of the structure-building components is white beeswax, which is natural beeswax but chemically bleached. It consists of esters of $\mathrm{C} 24$ to $\mathrm{C} 36$ straight-chain alcohols and straight-chain acids. It is used in creams, oils, ointments to 
increase consistency and in emulsions as a stabilizer. According to the literature, its melting point is between 60-67 ${ }^{\circ} \mathrm{C}$ [36]. In our investigation, DSC measurements show an endothermic peak at $64.45{ }^{\circ} \mathrm{C}$ (Fig. 2).

The other type of structure-building component is cetostearyl alcohol, which is a mixture of solid alcohols and consists of mainly stearyl and cetyl alcohols. It is mostly used in semisolids as a surfactant, a co-surfactant or as a viscosity increasing agent. In oral dosage forms it may slow the dissolution. The specific melting range of the substance is between $48-56{ }^{\circ} \mathrm{C}$ [36]. According to our DSC measurements, it is $56.61{ }^{\circ} \mathrm{C}$ (Fig. 2).

Kolliphor RH40 is a polyoxyethylene 40 castor oil derivative. It can be used as an emulsifying or wetting agent. According to the literature [36] and the DSC measurements, it has a congealing temperature at $16-26{ }^{\circ} \mathrm{C}$, and a melting point approximately at $30{ }^{\circ} \mathrm{C}$ (Fig. 2).

Suppocire BP was used as the lipophilic base of the systems. It has a melting range between $36-37{ }^{\circ} \mathrm{C}$ and a hydroxyl value between 30 and 50. Mostly, it is used as a base for suppositories [36]. According to our measurements, it has a melting point at $39.08^{\circ} \mathrm{C}$ (Fig. 2).

Our results show that the softening temperature of the systems meets our expectations as all of the systems soften partially between $34-36{ }^{\circ} \mathrm{C}$, which is slightly lower than the average body temperature in the oral cavity (Fig. 2), resulting in adaptation to the geometry of PPs.

Firstly, we evaluated how the concentration of the surface active agent affects the melting point of the compositions. The concentration of the structure-building component was fixed (WB at $10 \mathrm{~m} / \mathrm{m} \%$ or CA at $5 \mathrm{~m} / \mathrm{m} \%$ ). The effect of the increasing quantity of the surface active agent (from 5 $\mathrm{m} / \mathrm{m} \%$ to $20 \mathrm{~m} / \mathrm{m} \%$ ) on the melting point was examined. The graphs (Figs. 2 and 3 ) clearly show that the specific endothermic peak at the melting point of the structure-building component (WB: $64.45^{\circ} \mathrm{C}$, CA: $56.61{ }^{\circ} \mathrm{C}$ ) cannot be seen, which means that in this concentration range the structurebuilding components are incorporated homogeneously into the structure instead of the formation of a separated crystalline structure. Besides this, the $37^{\circ} \mathrm{C}$ melting point of SBP decreases and the sharp peak at $37^{\circ} \mathrm{C}$ broadens in the direction of lower temperatures, but the melting point stays above $35^{\circ} \mathrm{C}$.

Following this, the effect of the concentration of the structure-building component on the thermal behaviour of the formulations was evaluated. In this case, the concentration of the surface active agent was held at the mean value of $10 \mathrm{~m} / \mathrm{m} \%$, while the concentration of the structure-building component was changed from $5 \mathrm{~m} / \mathrm{m} \%$ (CA) or $10 \mathrm{~m} / \mathrm{m} \%$ (WB) to 20,40 and $60 \mathrm{~m} / \mathrm{m} \%$. It can be seen clearly that with the increasing concentration of the structure-building component, another peak appears above the temperature of $40{ }^{\circ} \mathrm{C}$, which corresponds to the melting point of waxes, but it is lower than the melting point of the pure material. The peak at the melting point of SBP shifted to lower temperatures when the concentration of the structure-building component was increased to $20 \mathrm{~m} / \mathrm{m} \%$ and shifted to higher temperatures when it was changed to 40 or $60 \mathrm{~m} / \mathrm{m} \%$. (Figs. 4 and 5).

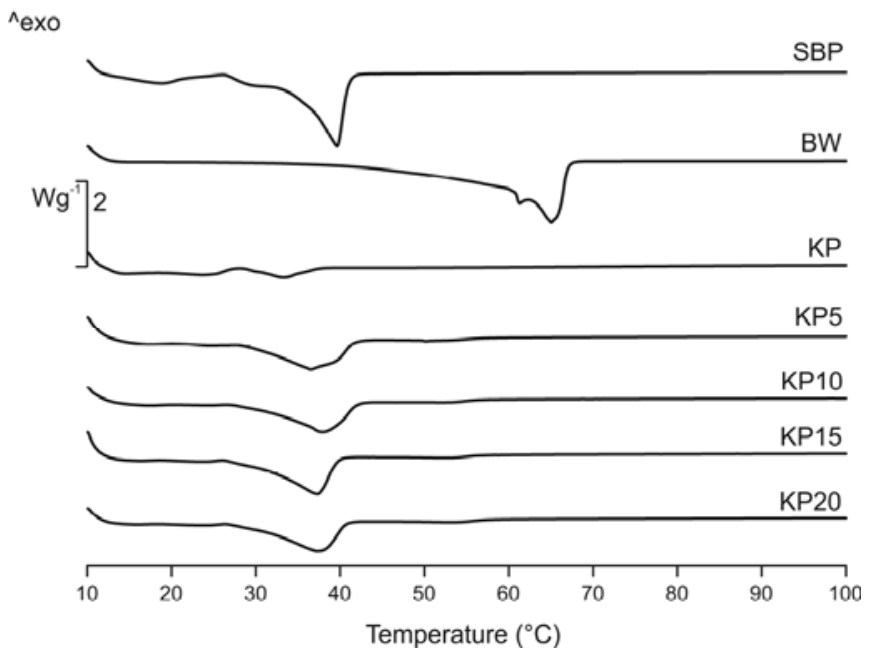

Fig. (2). DSC curves of pure Suppocire BP, white beeswax and Kolliphor RH40 and the compositions containing $10 \mathrm{~m} / \mathrm{m} \%$ white beeswax and 5,10,15 and $20 \mathrm{~m} / \mathrm{m} \%$ of surface active agent.

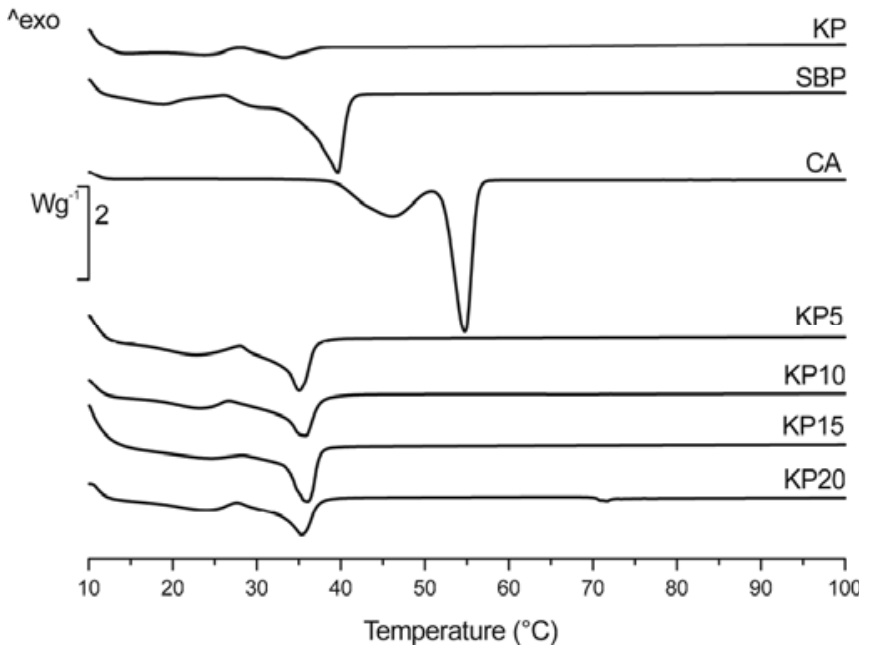

Fig. (3). DSC curves of pure Suppocire BP, cetostearyl alcohol, Kolliphor RH40 and the compositions containing $5 \mathrm{~m} / \mathrm{m} \%$ cetostearyl alcohol, 5, 10, 15 and $20 \mathrm{~m} / \mathrm{m} \%$ of surface active agent.

The DSC results (Figs. 4 and 5) clearly show that for formulations containing $20 \mathrm{~m} / \mathrm{m} \%$ of structure-building component there is no separate peak of the base and the structure building component, which will result in total melting at body temperature and sustained drug release probably cannot be reached thanks to the disintegration of the melted formulation.

The compositions containing 40 and $60 \mathrm{~m} / \mathrm{m} \%$ of the structure-building component show different results. The peaks of the structure-building component and the lipid base are separate, thus there is a compound with a lower melting point and another with a higher one (Figs. 4 and 5) From this it can be concluded that the system has a part which will melt or soften at body temperature, and another part which will give a structure to the system even at body temperature, thereby hindering disintegration and fast degradation.

\subsection{Wettability of the Delivery Systems}

As the systems are mostly hydrophobic, a surface active agent is needed to help the wetting of the delivery systems 
^exo

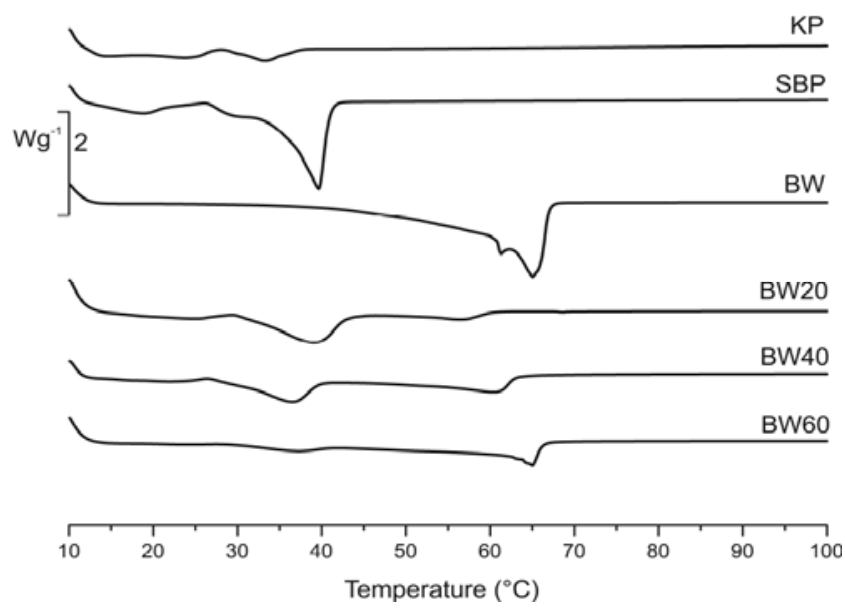

Fig. (4). DSC curves of pure Suppocire BP, white beeswax and Kolliphor RH40 and the compositions containing different concentrations $(20,40$ and $60 \mathrm{~m} / \mathrm{m} \%)$ of structure-building component (white beeswax).

^exo

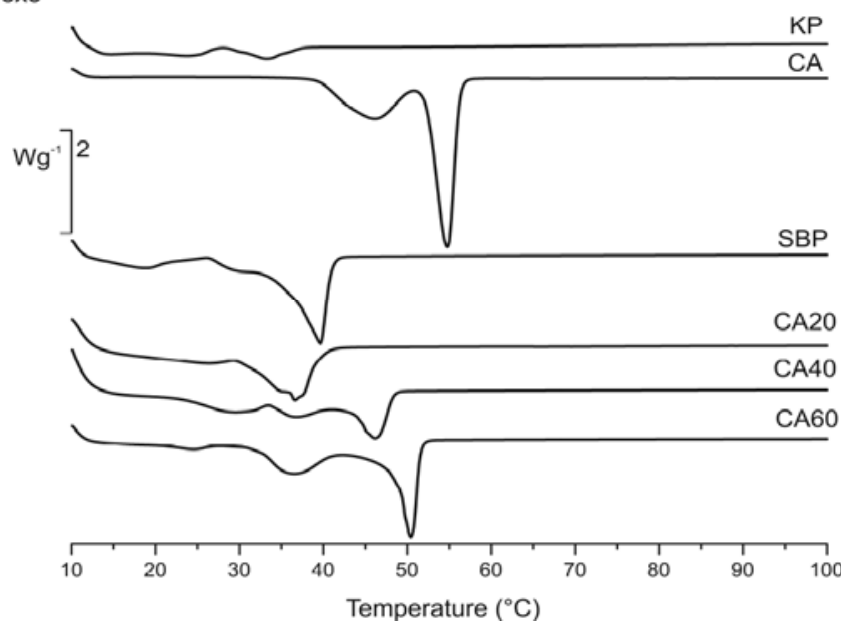

Fig. (5). DSC curves of pure Suppocire BP, cetostearyl alcohol and Kolliphor RH40 and the compositions containing different concentrations $(20,40$ and $60 \mathrm{~m} / \mathrm{m} \%)$ of structure-building component (cetostearyl alcohol).

with exudates in PPs, which is critical to the swelling of the preparations and consequently to drug release. Good wetting contributes to the degradation and elimination of the systems from the PP. An optical contact angle measurement system was used to determine the wettability of our systems.

First, we evaluated the effect of various concentrations of the surface active agent $(5,10,15$ and $20 \mathrm{~m} / \mathrm{m} \%)$, then we measured the effect of the structure-building components with constant surfactant concentrations.

In case of beeswax and the varying concentrations of surface active agent, the higher concentrations of surfactant decreased the contact angle, which means the wetting properties of the delivery systems became better. There is no remarkable difference between the preparations containing 10, 15 and 20 $\mathrm{m} / \mathrm{m} \%$ of surface active agent (Table 2). Based on the results of formulations containing WB, the best is the $10 \mathrm{~m} / \mathrm{m} \%$ concentration of surface active agent as the same wettability can be reached with higher amounts of wetting agent.

The evaluation of the results of the drug delivery systems containing $\mathrm{CA}$ as a structure-building component showed that the formulation with the best wettability was the one with $20 \mathrm{~m} / \mathrm{m} \%$ of surfactant (Table 2). Contact angles had a max. value at $15 \mathrm{~m} / \mathrm{m} \%$ of surface active agent, but the differences between the various compositions were not remarkable so the $10 \mathrm{~m} / \mathrm{m} \%$ of surfactant was chosen. All of the formulations made with CA (except the one with the $15 \mathrm{~m} / \mathrm{m}$ $\%$ of surfactant) had better wettability than the preparations containing WB.

The effect of the different concentrations of the structurebuilding component on the wettability of the preparations was measured. The concentration of the wetting agent was held at a $10 \mathrm{~m} / \mathrm{m} \%$ value (Table 3 ).

Increasing concentrations of WB increased wettability as the contact angle was smaller. The same is not applicable to the formulations containing CA. The contact angle increases with increasing the CA concentration (Table 3 ).

\subsection{Determination of Consistency by Penetrometry}

The consistency of the formulations was measured by penetrometry. The penetration depth of a penetrating object into a test sample was measured. From penetration depth consistency can be calculated or it directly refers to the consistency of the samples. The effect of the surface active agent and the structure-building component on consistency was evaluated.

Table 2. Average and SD of contact angles of compositions, made with white beeswax or cetostearyl alcohol, containing different concentrations $(5,10,15$ and $20 \mathrm{~m} / \mathrm{m} \%)$ of surface active agent.

\begin{tabular}{|c|c|c|c|c|}
\hline \multirow{3}{*}{ Concentration of KP $(\%)$} & \multicolumn{4}{|c|}{ Contact angle $\left(^{\circ}\right)$} \\
\hline & \multicolumn{2}{|c|}{ WB } & \multicolumn{2}{|c|}{ CA } \\
\hline & Average & SD & Average & SD \\
\hline 5 & 94.63 & 1.63 & 66.81 & 3.20 \\
\hline 10 & 98.04 & 4.10 & 75.44 & 8.72 \\
\hline 15 & 98.33 & 1.32 & 102.24 & 1.63 \\
\hline 20 & 100.86 & 0.65 & 58.29 & 6.12 \\
\hline
\end{tabular}


Table 3. Average and SD of contact angles of compositions containing different concentrations of the structure-building components (white beeswax or cetostearyl alcohol) compared to compositions with a lower quantity of structure-building component.

\begin{tabular}{|c|c|c|c|c|}
\hline \multirow{3}{*}{$\begin{array}{l}\text { Concentration of the Structure-building } \\
\text { Component }(\%)\end{array}$} & \multicolumn{4}{|c|}{ Contact Angle $\left(^{\circ}\right)$} \\
\hline & \multicolumn{2}{|c|}{ WB } & \multicolumn{2}{|c|}{$\mathbf{C A}$} \\
\hline & Average & SD & Average & SD \\
\hline 20 & 91.80 & 7.53 & 46.60 & 7.19 \\
\hline 40 & 76.27 & 11.12 & 60.95 & 4.15 \\
\hline 60 & 74.30 & 7.65 & 69.16 & 1.71 \\
\hline
\end{tabular}

Table 4. Average and SD of penetration into compositions, made with white beeswax or cetostearyl alcohol, containing different concentrations $(5,10,15$ and $20 \mathrm{~m} / \mathrm{m} \%)$ of surface active agent.

\begin{tabular}{|c|c|c|c|c|}
\hline \multirow{3}{*}{ Concentration of KP (\%) } & \multicolumn{4}{|c|}{ Penetration Depth (mm) } \\
\hline & \multicolumn{2}{|c|}{ WB } & \multicolumn{2}{|c|}{$\mathbf{C A}$} \\
\hline & Average & SD & Average & SD \\
\hline 5 & 5.63 & 0.25 & 4.34 & 0.07 \\
\hline 10 & 5.76 & 0.34 & 4.54 & 0.11 \\
\hline 15 & 5.79 & 0.27 & 4.87 & 0.20 \\
\hline 20 & 6.54 & 0.27 & 4.84 & 0.26 \\
\hline
\end{tabular}

Formulations made with CA were harder as penetration into the test objects was smaller. The concentration of the surface active agent had a small effect on penetration depth and consistency. The increasing concentration of KP caused deeper penetration into the penetrating objects except for the preparations made with CA containing $15 \mathrm{~m} / \mathrm{m} \%$ and 20 $\mathrm{m} / \mathrm{m} \%$ of KP, where the penetration depth was $0.03 \mathrm{~mm}$ deeper when measuring the system with a smaller quantity of surface active agent (Table 4).

As the surfactant concentration had not influenced the penetration depth remarkably, in accordance with the DSC and wettability results the formulations with $10 \mathrm{~m} / \mathrm{m} \%$ of surface active agent was chosen to carry out the further investigations.

The penetration depth into preparations made with different concentrations of structure-building component was also evaluated. The concentration of the surface active agent was held at a constant $10 \mathrm{~m} / \mathrm{m} \%$ value. The increasing quantity of the structure-building component in case of both WB and $\mathrm{CA}$ made the formulations harder and the penetration depth shallower. CA had a greater impact on penetration depth and consistency than WB because 40 and $60 \mathrm{~m} / \mathrm{m} \%$ concentrations showed a higher decrease in penetration depth (Table 5).

The formulations containing CA have better wettability and solidity, which may influence not only the application of the system but also drug release.

\subsection{Swelling and Degradation}

The swelling and degradation properties of formulations containing 20, 40 and $60 \mathrm{~m} / \mathrm{m} \%$ of CA were measured gravimetrically to determine whether the preparations are able to absorb water, swell and degrade. Degradation is necessary as the systems are aimed to be biodegradable and to be eliminated from the subgingival area.

The formulations containing $20 \mathrm{~m} / \mathrm{m} \%$ of structurebuilding component fell apart on the first day of measurement. In light of this, the results connected to $20 \mathrm{~m} / \mathrm{m} \%$ of $\mathrm{CA}$ are not shown in the figures.

Fig. (6) shows the swelling ratio of the formulations containing 40 and $60 \mathrm{~m} / \mathrm{m} \%$ of CA.

Swelling ratio was calculated as follows:

Swelling ratio $=\frac{m_{s}}{m_{d}}$

where $m_{s}$ is the mass of the formulations after immersed in water for the specified time and $m_{d}$ is the mass of the dried formulations.

The results show that the preparations containing $40 \mathrm{~m} / \mathrm{m}$ $\%$ of structure-building component can absorb more water as they are not as hard as the formulations containing $60 \mathrm{~m} / \mathrm{m}$ $\%$ of CA (Fig. 6).

Swelling lasts for four days, then the swelling ratio remains on the same level. This may happen because a balance is formed between swelling and degradation (Fig. 6). 
Table 5. Average and SD of penetration into compositions containing different concentrations of the structure-building components (white beeswax or cetostearyl alcohol) compared to compositions with a lower quantity of structure-building component.

\begin{tabular}{|c|c|c|c|c|}
\hline \multirow{3}{*}{$\begin{array}{l}\text { Concentration of the Structure-building } \\
\text { Component (\%) }\end{array}$} & \multicolumn{4}{|c|}{ Penetration Depth $(\mathrm{mm})$} \\
\hline & \multicolumn{2}{|c|}{ WB } & \multicolumn{2}{|c|}{$\mathbf{C A}$} \\
\hline & Average & SD & Average & SD \\
\hline 20 & 4.85 & 0.12 & 4.95 & 0.26 \\
\hline 40 & 4.35 & 0.15 & 3.17 & 0.27 \\
\hline 60 & 3.60 & 0.23 & 1.87 & 0.09 \\
\hline
\end{tabular}

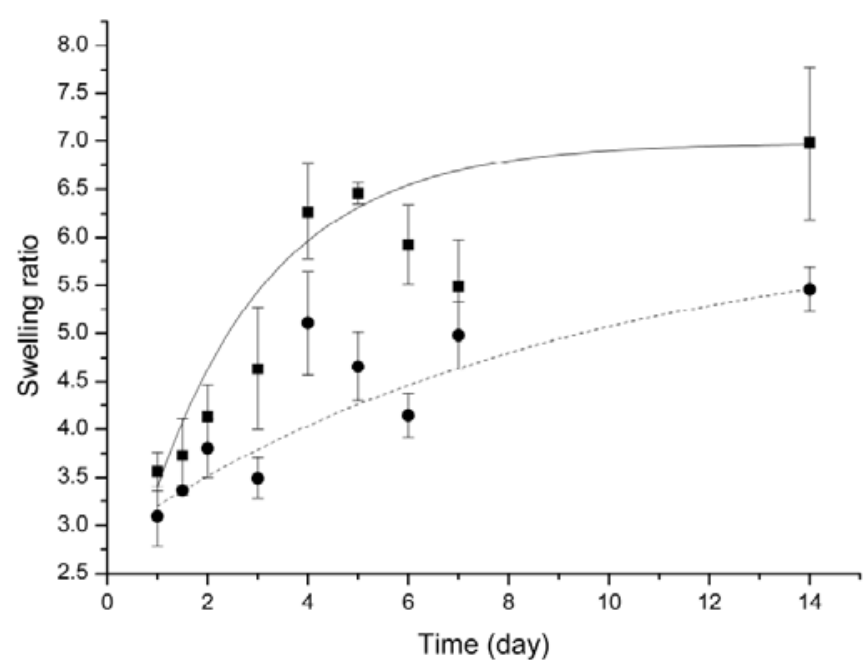

Fig. (6). Swelling ratio of compositions containing 40 and $60 \mathrm{~m} / \mathrm{m}$ $\%$ of cetostearyl alcohol. $40 \mathrm{~m} / \mathrm{m} \%, \bullet 60 \mathrm{~m} / \mathrm{m} \%$ of cetostearyl alcohol. tion:

Degradation (\%) was calculated from the following equa-

Degradation $(\%)=\frac{m_{o}-m_{d}}{m_{o}} \cdot 100 \%$

where $m_{o}$ is the mass of the original formulations and $m_{d}$ is the mass of the dried formulation after the swelling and degradation measurements.

Degradation properties show no remarkable differences between the two concentrations. Similarly to swelling, the increase of degradation stops on the fourth day as a possible result of a balance between swelling and degradation (Fig. 7).

\subsection{Drug Release Study}

Drug release measurements were performed to investigate the possibility of achieving the sustained release of active agents by using increasing concentrations of the structure-building component. Both of the structure-building agents were tested. The drug release of two different antimicrobials (amoxicillin and metronidazole) was evaluated from the compositions.

AMX is a semi-synthetic penicillin derivative, with a broad spectrum of antibiotic activity against aerobic or anaerobic Gram-positive and Gram-negative bacteria [18, 37].

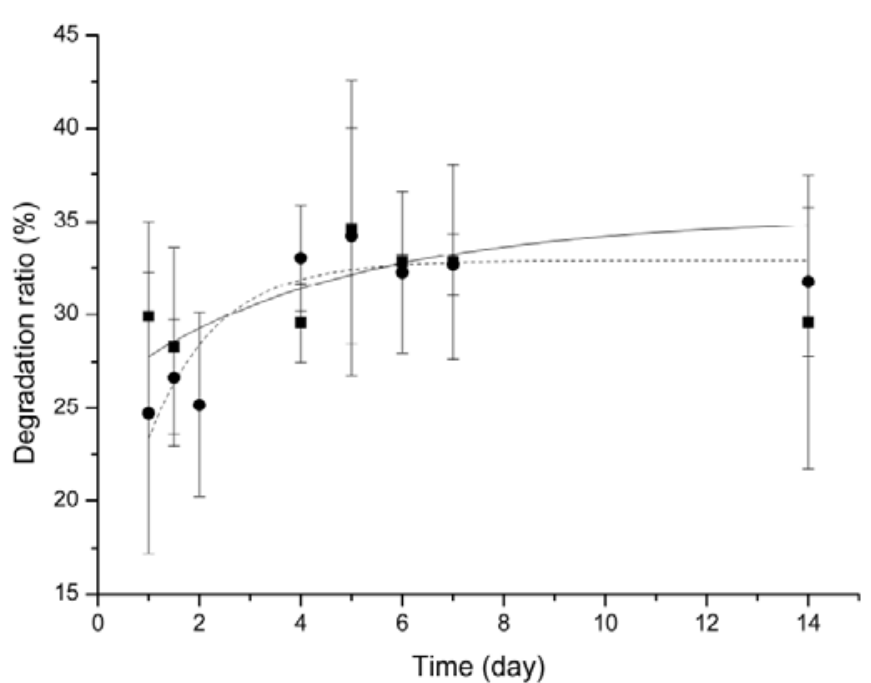

Fig. (7). Degradation of compositions containing 40 and $60 \mathrm{~m} / \mathrm{m} \%$ of cetostearyl alcohol. - $40 \mathrm{~m} / \mathrm{m} \%, \bullet 60 \mathrm{~m} / \mathrm{m} \%$ cetostearyl alcohol.

$\mathrm{MZ}$ is an antibacterial agent, belonging to the nitroimidazole group. It is known to be effective against numerous bacteria and protozoa, including anaerobes $[2,38]$.

$\mathrm{MZ}$ and AMX are also used in the therapy of periodontal disease as an adjunct to the mechanical debridement (scaling, root planing) locally or systematically [39].

Six different compositions were measured and with every composition 3 parallel measurements were carried out. The release of MZ was investigated from systems made with WB and CA. AMX release was only tested for preparations with $\mathrm{CA}$ due to the results of $\mathrm{MZ}$ release. The formulations contained: SBP, Methocel E4M, KP, 20, 40 and $60 \mathrm{~m} / \mathrm{m} \%$ of $\mathrm{WB}$ or $\mathrm{CA}, 10 \mathrm{~m} / \mathrm{m} \%$ of AMX or MZ.

The results of the evaluation of $\mathrm{MZ}$ release from formulations containing 20, 40 and $60 \mathrm{~m} / \mathrm{m} \%$ of WB showed large $\mathrm{SD}$ values. This may be due to the fact that the compositions fell apart during the drug release tests. It can be concluded that WB is unsuitable for providing the sustained release of antimicrobial agents, so because of this AMX release was measured only from formulations made with CA.

The results show that, with increasing concentrations of $\mathrm{CA}$, it is possible to prolong the MZ release from the formulations (Fig. 8). Fig. (8) shows that formulations containing 


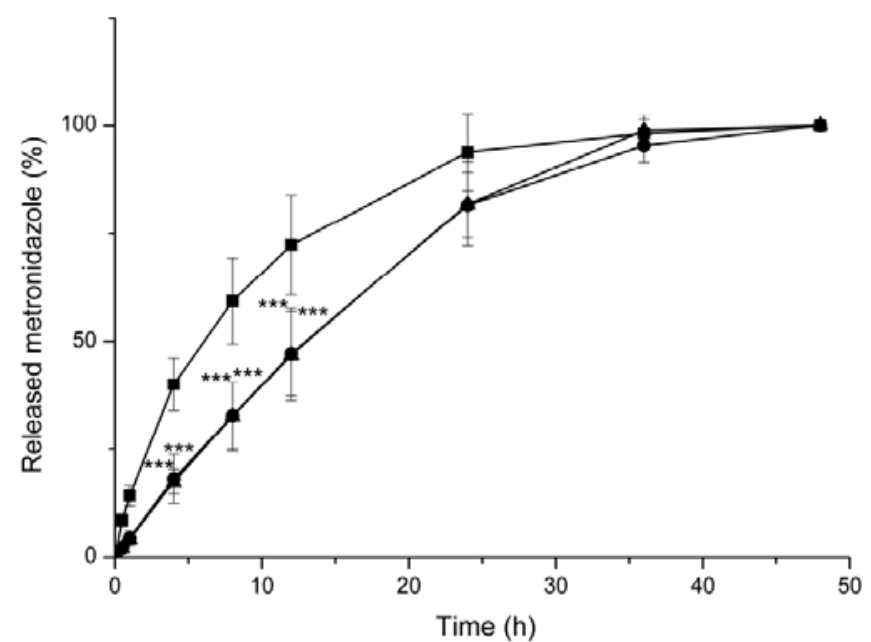

Fig. (8). Release of metronidazole from formulations containing different concentrations $(20,40$ and $60 \mathrm{~m} / \mathrm{m} \%)$ of cetostearyl alcohol. - $20 \mathrm{~m} / \mathrm{m} \%, \bullet 40 \mathrm{~m} / \mathrm{m} \%, \boldsymbol{\Delta} 60 \mathrm{~m} / \mathrm{m} \%$ cetostearyl alcohol. Drug release from compositions containing 40 and $60 \mathrm{~m} / \mathrm{m} \%$ cetostearyl alcohol shows highly significant difference $(* * *)$ compared to formulations with $20 \mathrm{~m} / \mathrm{m} \%$ cetostearyl alcohol at 4, 8 and 12 hours.

$20 \mathrm{~m} / \mathrm{m} \%$ of CA have a faster drug release than the formulations made with 40 or $60 \mathrm{~m} / \mathrm{m} \%$ of CA, which can be explained by the faster degradation of formulations containing $20 \mathrm{~m} / \mathrm{m} \%$ CA during the drug release measurements. There is no significant difference between the systems containing 40 or $60 \mathrm{~m} / \mathrm{m} \%$ of structure-building components.

AMX release from formulations made with CA was similar to the release of MZ as it was possible to sustain the AMX release. Another similarity to MZ release is that there is no significant difference between the release of AMX from formulations made with $40 \mathrm{~m} / \mathrm{m} \%$ and $60 \mathrm{~m} / \mathrm{m} \%$ CA (Fig. 9).

The drug release mechanism can be characterized with the following equation:

$\frac{M_{t}}{M_{\alpha}}=k t^{n}$

where $M_{t} / M_{\propto}$ is the fraction of drug released, $k$ is the kinetic constant and $n$ is the release exponent describing the mechanism of the release [40].

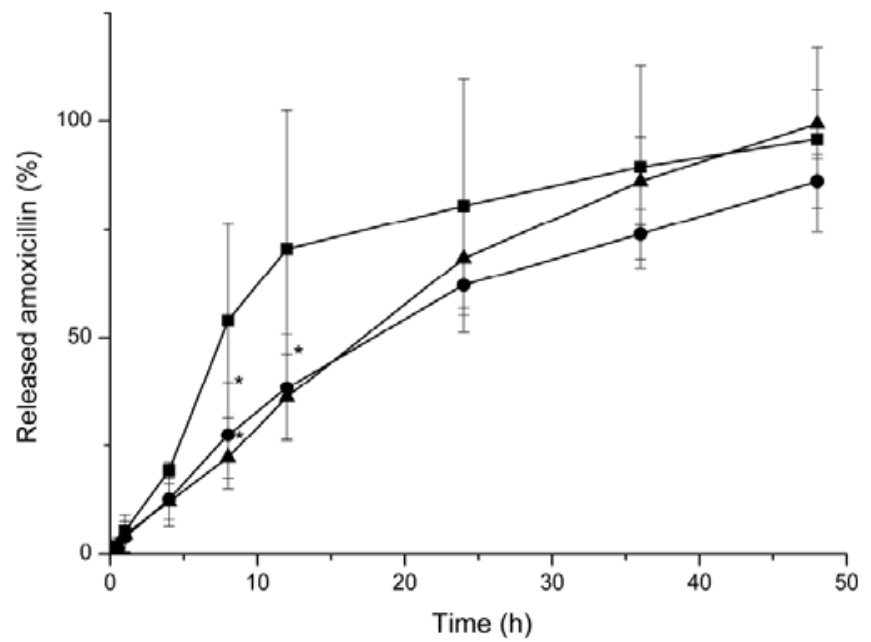

Fig. (9). Release of amoxicillin from formulations containing different concentrations $(20,40$ and $60 \mathrm{~m} / \mathrm{m} \%)$ of cetostearyl alcohol. - $20 \mathrm{~m} / \mathrm{m} \%, \bullet 40 \mathrm{~m} / \mathrm{m} \%, \boldsymbol{\Delta} 60 \mathrm{~m} / \mathrm{m} \%$ cetostearyl alcohol. Drug release from compositions containing $40 \mathrm{~m} / \mathrm{m} \%$ cetostearyl alcohol shows significant difference at $(*)$ at 8 hours compared to formulations with $20 \mathrm{~m} / \mathrm{m} \%$ cetostearyl alcohol. The drug release from compositions containing $60 \mathrm{~m} / \mathrm{m} \%$ cetostearyl alcohol also shows significant difference $(*)$ at 8 and 12 hours compared to formulations with $20 \mathrm{~m} / \mathrm{m} \%$ cetostearyl alcohol.

These values were determined from the equation of drug release curves (Table $\mathbf{6}$ ).

\subsection{Investigation of Sustained Antimicrobial Effect: Pre- liminary Study}

A preliminary microbiological study was made to investigate the sustained microbiological activity of our formulations containing 5, 10 and $15 \mathrm{~m} / \mathrm{m} \%$ of AMX. The results are in Table 7. The diameter of the inhibition zone was measured in $\mathrm{mm}$ and it is the average of two perpendicular diameters. The averages were rounded to integer numbers.

AMX containing systems inhibited bacterial growth for 8 to 21 days.

With $5 \mathrm{~m} / \mathrm{m} \%$ of AMX incorporated into the systems, growth inhibition lasted for 8 days. $10 \mathrm{~m} / \mathrm{m} \%$ of AMX caused a 10-day growth inhibition, while the $15 \mathrm{~m} / \mathrm{m} \%$ one lasted for 21 days. The inhibition zone of the $15 \mathrm{~m} / \mathrm{m} \%$ AMX contain-

Table 6. Release exponent $(n)$ and kinetic constant $(k)$ at different structure-building component concentrations of formulations containing amoxicillin or metronidazole and the $R^{2}$ values of the fitted curves.

\begin{tabular}{|c|c|c|c|c|}
\hline \multirow{2}{*}{ Active Ingredient } & $\begin{array}{c}\text { Structure-building Component } \\
\text { Concentration (m/m \%) }\end{array}$ & $\boldsymbol{n}$ & $\boldsymbol{k}$ \\
\hline \hline \multirow{3}{*}{ MZ } & 20 & 0.5560 & 15.169 & 0,9598 \\
\cline { 2 - 5 } & 40 & 0.8717 & 4.5846 & 0,9853 \\
\cline { 2 - 5 } & 60 & 0.8808 & 4.4657 & 0,9866 \\
\hline \multirow{2}{*}{ AMX } & 20 & 0,8503 & 5,4502 & 0,9416 \\
\cline { 2 - 5 } & 40 & 0,9264 & 3,1313 & 0,9721 \\
\cline { 2 - 5 } & 60 & 0,8457 & 4,1243 & 0,9955 \\
\hline
\end{tabular}


Table 7. Results of the preliminary microbiological investigations of formulations containing 5,10 and $15 \mathrm{~m} / \mathrm{m} \%$ of amoxicillin (AMX).

\begin{tabular}{|c|c|c|c|}
\hline \multirow{2}{*}{ Time (day) } & \multicolumn{3}{|c|}{ Diameter of Inhibition Zones (mm) } \\
\hline & $5 \%$ AMX & $10 \%$ AMX & $15 \%$ AMX \\
\hline Day 1 & 60 & 60 & no growth \\
\hline Day 2 & 60 & 60 & 60 \\
\hline Day 3 & 60 & 60 & 60 \\
\hline Day 6 & 50 & 54 & 60 \\
\hline Day 7 & 40 & 54 & 60 \\
\hline Day 8 & 20 & 42 & 58 \\
\hline Day 9 & 0 & 38 & 54 \\
\hline Day 10 & 0 & 16 & 52 \\
\hline Day 13 & 0 & 0 & 51 \\
\hline Day 14 & 0 & 0 & 50 \\
\hline Day 15 & 0 & 0 & 42 \\
\hline Day 16 & 0 & 0 & 40 \\
\hline Day 20 & 0 & 0 & 40 \\
\hline Day 21 & 0 & 0 & 18 \\
\hline Day 22 & 0 & 0 & 0 \\
\hline
\end{tabular}

ing formulation could not be measured after $24 \mathrm{~h}$ as there was no detectable growth of the bacteria in the Petri dish. The largest measurable inhibition zone was $60 \mathrm{~mm}$ in diameter and was smaller and smaller day by day (Table 7 ).

The shrinking zones are the signs of the decreasing concentrations of the active ingredient in the formulations as the systems were put on a new plate in every $24 \mathrm{~h}$.

During the microbiological evaluation all of the formulations absorbed water, swelled. Water absorption and swelling are necessary for drug release.

The prolonged antibacterial effect means that our delivery system is ready to sustain the AMX release (Table 7).

\section{CONCLUSION}

By developing lipid formulations, anhydrous drug delivery systems could be prepared which soften at body temperature, therefore they fit the geometry of the periodontal pockets. Wettability is provided by the added surface active agent, while the softening at body temperature is due to the low melting point of the lipid components $\left(35-37^{\circ} \mathrm{C}\right)$. Sustained drug release is supported by the structure-building components.

According to the wettability measurements, the $10 \mathrm{~m} / \mathrm{m}$ $\%$ Kolliphor RH40 concentration is optimal. The type and the concentration of the structure-building component highly influence hardness and - under $40 \mathrm{~m} / \mathrm{m} \%$ - drug release. The results of penetrometry, the swelling, degradation and drug release measurements indicate that the optimal structure- building component is CA in $40 \mathrm{~m} / \mathrm{m} \%$. In this composition the formula softens at body temperature and prolonged drug release can be ensured as well. Sustained drug release was also confirmed with a preliminary antimicrobial test.

In our work, we formulated a subgingival, swellable, biodegradable drug delivery system, which could provide sustained antimicrobial activity up to almost 3 weeks.

\section{CONSENT FOR PUBLICATION}

Not applicable.

\section{CONFLICT OF INTEREST}

The authors declare no conflict of interest, financial or otherwise.

\section{ACKNOWLEDGEMENTS}

We would like to express our gratitude to Gattefossé and BASF for providing us with Suppocire BP pellets and Kolliphor RH40.

\section{REFERENCES}

[1] A. Kopytynska-Kasperczyk, P. Dobrzynski, M. Pastusiak, B. Jarzabek, and W. Prochwicz, "Local delivery system of doxycycline hyclate based on -caprolactone copolymers for periodontitis treatment," Int. J. Pharm., vol. 491, no. 1-2, pp. 335344, 2015.

[2] K. Schwach-Abdellaoui, "Local delivery of antimicrobial agents for the treatment of periodontal diseases," Eur. J. Pharm. Biopharm., vol. 50, no. 1, pp. 83-99, Jul. 2000. 
[3] A. D. Haffajee and S. S. Socransky, "Microbial etiological agents of destructive periodontal diseases," Periodontol. 2000, vol. 5, no. 1, pp. 78-111, Jun. 1994.

[4] M. G. Newman, H. H. Takei, P. R. Klokkevold, and F. A. Carranza, Carranza's Clinical Periodontology. Elsevier Saunders, 2014.

[5] R. C. PAGE and K. S. KORNMAN, "The pathogenesis of human periodontitis: an introduction," Periodontol. 2000, vol. 14, no. 1, pp. 9-11, Jun. 1997.

[6] M. Costalonga and M. C. Herzberg, "The oral microbiome and the immunobiology of periodontal disease and caries," Immunol. Lett., vol. 162, no. 2, pp. 22-38, Dec. 2014.

[7] J. W. Costerton, "Bacterial Biofilms: A Common Cause of Persistent Infections," Science (80-. )., vol. 284, no. 5418, pp. 1318-1322, May 1999.

[8] G. Matuliene, B. E. Pjetursson, G. E. Salvi, K. Schmidlin, U. Brägger, M. Zwahlen, and N. P. Lang, "Influence of residual pockets on progression of periodontitis and tooth loss: Results after 11 years of maintenance," J. Clin. Periodontol., vol. 35, no. 8, pp. 685-695, 2008.

[9] P. D. Marsh, A. Moter, and D. A. Devine, "Dental plaque biofilms: communities, conflict and control," Periodontol. 2000, vol. 55, no. 1, pp. 16-35, Feb. 2011.

[10] E. Westfelt, "Rationale of mechanical plaque control.," J. Clin. Periodontol., vol. 23, no. Page 1986, pp. 263-267, 1996.

[11] K. Jepsen and S. Jepsen, “Antibiotics/antimicrobials: systemic and local administration in the therapy of mild to moderately advanced periodontitis," Periodontol. 2000, vol. 71, no. 1, pp. 82-112, Jun. 2016.

[12] M. P. Do, C. Neut, H. Metz, E. Delcourt, J. Siepmann, K. Mäder, and F. Siepmann, "Mechanistic analysis of PLGA/HPMC-based insitu forming implants for periodontitis treatment," Eur. J. Pharm. Biopharm., vol. 94, pp. 273-283, 2015.

[13] M. P. Do, C. Neut, H. Metz, E. Delcourt, K. Mäder, J. Siepmann, and F. Siepmann, "In-situ forming composite implants for periodontitis treatment: How the formulation determines system performance," Int. J. Pharm., vol. 486, no. 1-2, pp. 38-51, 2015.

[14] R. K. Agarwal, D. H. Robinson, G. I. Maze, and R. A. Reinhardt, "Development and characterization of tetracyclinepoly(lactide/glycolide) films for the treatment of periodontitis," $J$. Control. Release, vol. 23, no. 2, pp. 137-146, 1993.

[15] L. E. Bromberg, V. M. Braman, D. M. Rothstein, P. Spacciapoli, S. M. O'Connor, E. J. Nelson, D. K. Buxton, M. S. Tonetti, and P. M. Friden, "Sustained release of silver from periodontal wafers for treatment of periodontitis," J. Control. Release, vol. 68, no. 1, pp. 63-72, 2000

[16] M. L. Bruschi, D. S. Jones, H. Panzeri, M. P. D. Gremião, O. de Freitas, and E. H. G. Lara, "Semisolid Systems Containing Propolis for the Treatment of Periodontal Disease: In vitro Release Kinetics, Syringeability, Rheological, Textural, and Mucoadhesive Properties," J. Pharm. Sci., vol. 96, no. 8, pp. 2074-2089, Aug. 2007.

[17] D. P. Chinta, P. Katakam, V. S. N. Murthy, and M. J. Newton, "Formulation and in-vitro evaluation of moxifloxacin loaded crosslinked chitosan films for the treatment of periodontitis," $J$. Pharm. Res., vol. 7, no. 6, pp. 483-490, 2013.

[18] N. Jain, G. K. Jain, S. Javed, Z. Iqbal, S. Talegaonkar, F. J. Ahmad, and R. K. Khar, "Recent approaches for the treatment of periodontitis," Drug Discov. Today, vol. 13, no. 21-22, pp. 932943, 2008.

[19] B. S. Lee, C. C. Lee, H. P. Lin, W. A. Shih, W. L. Hsieh, C. H. Lai, Y. Takeuchi, and Y. W. Chen, "A functional chitosan membrane with grafted epigallocatechin-3-gallate and lovastatin enhances periodontal tissue regeneration in dogs," Carbohydr. Polym., vol. 151, pp. 790-802, 2016.

[20] M. Reise, R. Wyrwa, U. Müller, M. Zylinski, A. Völpel, M. Schnabelrauch, A. Berg, K. D. Jandt, D. C. Watts, and B. W. Sigusch, "Release of metronidazole from electrospun poly(1lactide-co-d/l-lactide) fibers for local periodontitis treatment," Dent. Mater., vol. 28, no. 2, pp. 179-188, 2012.

[21] G. L. Southard and K. C. Godowski, "Subgingival controlled release of antimicrobial agents in the treatment of periodontal disease.," Int. J. Antimicrob. Agents, vol. 9, no. 4, pp. 239-253, 1998.
[22] S. C. Sundararaj, M. V. Thomas, T. D. Dziubla, and D. A. Puleo, "Bioerodible system for sequential release of multiple drugs," Acta Biomater., vol. 10, no. 1, pp. 115-125, 2014.

[23] S. C. Sundararaj, M. V. Thomas, R. Peyyala, T. D. Dziubla, and D. A. Puleo, "Design of a multiple drug delivery system directed at periodontitis," Biomaterials, vol. 34, no. 34, pp. 8835-8842, 2013.

[24] M. Yar, A. Farooq, L. Shahzadi, A. S. Khan, N. Mahmood, A. Rauf, A. A. Chaudhry, and I. U. Rehman, "Novel meloxicam releasing electrospun polymer/ceramic reinforced biodegradable membranes for periodontal regeneration applications," Mater. Sci. Eng. C, vol. 64, pp. 148-156, 2016.

[25] M. P. Do, C. Neut, E. Delcourt, T. Seixas Certo, J. Siepmann, and F. Siepmann, "In situ forming implants for periodontitis treatment with improved adhesive properties," Eur. J. Pharm. Biopharm., vol. 88 , no. 2 , pp. 342-350, 2014.

[26] M. Y. Al-Saeed and N. Babay, "The use of povidone-iodine and hydrogen peroxide mixture as an adjunct to non-surgical treatment of slight to moderate chronic periodontitis," Saudi Dent. J., vol. 21, no. 3, pp. 127-133, 2009.

[27] M. P. and R. S. Dhesingh*, "Nanoparticle Modified Drug Loaded Biodegradable Polymeric Contact Lenses for Sustainable Ocular Drug Delivery," Current Drug Delivery, vol. 14, no. 4. pp. 555$565,2017$.

[28] B. L. Li, M. I. Setyawati, L. Chen, J. Xie, K. Ariga, C.-T. Lim, S. Garaj, and D. T. Leong, "Directing Assembly and Disassembly of 2D MoS2 Nanosheets with DNA for Drug Delivery," ACS Appl. Mater. Interfaces, vol. 9, no. 18, pp. 15286-15296, May 2017.

[29] L. Wang, X. Yang, Y. Di, K. Chen, and H. W. and W. Pan, "ZeroOrder Controlled Delivery of Gliclazide from Polyethylene Oxides Matrix Tables: In vitro and In vivo Evaluation," Current Drug Delivery, vol. 14, no. 1. pp. 136-144, 2017.

[30] A. Paudel, Ameeduzzafar, S. S. Imam, M. Fazil, S. Khan, A. Hafeez, and F. J. A. and A. Ali*, "Formulation and Optimization of Candesartan Cilexetil Nano Lipid Carrier: In vitro and In vivo Evaluation," Current Drug Delivery, vol. 14, no. 7. pp. 1005-1015, 2017.

[31] A. L. C. Maia, C. Fernandes, C. N. P. de Oliveira, C. S. Teixeira, M. S. Oliveira, and D. C. F. S. and G. A. Ramaldes, "Liposomes Containing Gadodiamide: Preparation, Physicochemical Characterization, and In vitro Cytotoxic Evaluation," Current Drug Delivery, vol. 14, no. 4. pp. 566-574, 2017.

[32] M. Komiyama, K. Yoshimoto, M. Sisido, and K. Ariga, "Chemistry Can Make Strict and Fuzzy Controls for Bio-Systems: DNA Nanoarchitectonics and Cell-Macromolecular Nanoarchitectonics," Bull. Chem. Soc. Jpn., vol. 90, no. 9, pp. 9671004, Sep. 2017.

[33] X. Li, J. Tsibouklis, T. Weng, B. Zhang, G. Yin, G. Feng, Y. Cui, I. N. Savina, L. I. Mikhalovska, S. R. Sandeman, C. A. Howel, and S. V. Mikhalovsky, "Nano carriers for drug transport across the blood-brain barrier," J. Drug Target., vol. 25, no. 1, pp. 17-28, Jan. 2017.

[34] Y. Koseki, Y. Ikuta, T. Kamishima, T. Onodera, H. Oikawa, and H. Kasai, "Drug Release is Determined by the Chain Length of Fatty Acid-Conjugated Anticancer Agent as One Component of NanoProdrug," Bull. Chem. Soc. Jpn., vol. 89, no. 5, pp. 540-545, May 2016.

[35] M. R. C. Marques, R. Loebenberg, and M. Almukainzi, "Simulated Biological Fluids with Possible Application in Dissolution Testing," Dissolution Technol., vol. 18, no. 3, pp. 15-28, 2011.

[36] R. C. Rowe, P. J. Sheskey, and M. E. Quinn, Handbook of Pharmaceutical Excipients, 6th editio. Pharmaceutical Press, 2009.

[37] R. Sutherland, E. a Croydon, and G. N. Rolinson, "Amoxycillin: a new semi-synthetic penicillin.," Br. Med. J., vol. 3, no. 5817, pp. 13-16, 1972.

[38] D. I. Edwards, "Mechanisms of selective toxicity of metronidazole and other nitroimidazole drugs.," Br. J. Vener. Dis., vol. 56, no. 5, pp. 285-290, Oct. 1980.

[39] N. Cionca, C. Giannopoulou, G. Ugolotti, and A. Mombelli, "Amoxicillin and metronidazole as an adjunct to full-mouth scaling and root planing of chronic periodontitis.," J. Periodontol., vol. 80, no. 3, pp. 364-371, 2009.

[40] N. A. Peppas, P. Bures, W. Leobandung, and H. Ichikawa, "Hydrogels in pharmaceutical formulations," Eur. J. Pharm. Biopharm., vol. 50, no. 1, pp. 27-46, 2000. 


$$
\text { II. }
$$




\title{
Combination of Zinc Hyaluronate and Metronidazole in a Lipid-Based Drug Delivery System for the Treatment of Periodontitis
}

\author{
Attila Léber ${ }^{1}$, Mária Budai-Szúcs ${ }^{1}$, Edit Urbán ${ }^{2}$, Péter Vályi ${ }^{3}{ }^{\circledR}$, Attila Gácsi ${ }^{1}$, Szilvia Berkó ${ }^{1}$, \\ Anita Kovács ${ }^{1}$ and Erzsébet Csányi ${ }^{1, *(D)}$ \\ 1 Institute of Pharmaceutical Technology and Regulatory Affairs, Faculty of Pharmacy, University of Szeged, \\ Szeged 6720, Hungary; leber.attila@pharm.u-szeged.hu (A.L.); maria.szucs@pharm.u-szeged.hu (M.B.-S.); \\ gacsi.attila@pharm.u-szeged.hu (A.G.); berkosz@pharm.u-szeged.hu (S.B.); \\ anita.kovacs@pharm.u-szeged.hu (A.K.) \\ 2 Institute of Clinical Microbiology, Faculty of Medicine, University of Szeged, Szeged 6720, Hungary; \\ tidenabru@freemail.hu \\ 3 Department of Periodontology, Faculty of Dentistry, University of Szeged, Szeged 6720, Hungary; \\ valyi.peter@stoma.u-szeged.hu \\ * Correspondence: csanyi@pharm.u-szeged.hu; Tel.: +36-62-545-573
}

Received: 11 February 2019; Accepted: 21 March 2019; Published: 25 March 2019

\begin{abstract}
Background: Despite being a highly prevalent disease and a possible contributor to adult tooth loss, periodontitis possesses no well-established therapy. The aim of the recent study was the development and evaluation of a mucoadhesive monophase lipid formulation for the sustained local delivery of amoxicillin, metronidazole, and/or zinc hyaluronate or gluconate. Methods: To investigate our formulations, differential scanning calorimetry, $\mathrm{X}$-ray diffraction, swelling, erosion, mucoadhesivity, drug release, and antimicrobial measurements were performed. Results: Differential scanning calorimetry (DSC) and X-ray diffraction (XRD) results show that the loaded drugs are in a suspended form, the softening of the formulations starts at body temperature, but a part remains solid, providing sustained release. Swelling of the lipid compositions is affected by the hydrophilic components, their concentration, and the strength of the coherent lipid structure, while their erosion is impacted by the emulsification of melted lipid components. Conclusions: Results of drug release and antimicrobial effectiveness measurements show that a sustained release may be obtained. Amoxicillin had higher effectiveness against oral pathogens than metronidazole or zinc hyaluronate alone, but the combination of the two latter could provide similar effectiveness to amoxicillin. The applied mucoadhesive polymer may affect adhesivity, drug release through the swelling mechanism, and antimicrobial effect as well.
\end{abstract}

Keywords: periodontitis; lipid drug delivery; zinc hyaluronate; amoxicillin; metronidazole

\section{Introduction}

The plaque-induced forms of periodontal diseases are the most prevalent chronic inflammatory conditions seen in humans worldwide, affecting nearly half of the adults. Periodontitis is a major health problem reducing the quality of life. Not only does it cause tooth loss, disability, masticatory dysfunction, poor nutritional status, and compromised speech, but it is also independently associated with systemic chronic inflammatory diseases including atherogenic cardiovascular disease, type 2 diabetes mellitus, rheumatoid arthritis, chronic kidney disease, obesity, and chronic obstructive pulmonary disease [1-3]. 
Periodontitis is predominantly a bacterial infection involving the accumulation of different bacteria on the non-shedding surfaces. In susceptible patients, dental plaque comprises periodontal pathogenic microorganisms which initiate and trigger a dysfunctional inflammatory immune response, which results in the destruction of the underlying supporting tissues. The host response to the initiating periodontopathogens is mostly a genetically determined non-modifiable risk factor. However, modifiable or eliminative risk factors play an important role in changing the susceptibility or resistance of individuals to the disease. Such risk factors are smoking, poorly controlled diabetes mellitus, obesity, stress, osteopenia, and inadequate intake of calcium and vitamin D. [1]. The most important modifiable local risk factor is poor oral hygiene, which may initiate the infective inflammatory process in periodontal tissues.

Treatment of periodontitis involves a fine balance of various non-surgical and surgical methods carried out in order to reduce periodontal pocket depth, access residual plaque, initiate the regeneration of periodontal supporting tissues and decrease the risk of disease progression. Subgingival antiinfective therapy performed together with self-performed plaque control provides significant benefits in clinical parameters and improvement of systemic inflammatory markers [4].

Non-surgical periodontal treatment consists of professional removal of plaque and calculus, elimination of plaque retentive factors, oral hygiene instruction, chemical plaque control and antibiotic medication. Treatment of teeth with tooth/site-dependent factors (e.g., the presence of plaque, anatomic features, deep pocket, furcation involvement, intrabony defect) is less predictable and bears an elevated risk of the progression of the breakdown of periodontal tissues [5]. Mechanical therapy alone may have limited effect on some periodontopathogens and fail to eliminate them in ecological niches (e.g., infected epithelial and connective tissue pocket wall, scratches left after mechanical debridement, resorption lacunae, accessory root canal, dentinal tubules, dorsal part of the tongue, tonsils, oral mucosa, etc.). These limitations indicate the importance of the employment of pharmacologic agents in periodontal therapy [4].

Pharmacologic therapies are based on the administration of antimicrobials (including antiseptics and local delivery or systemic regimen of antibiotics) and probiotics and host modulation. Systemic antimicrobials should be applied adjunctively to mechanical non-surgical treatment—preferably in patients with aggressive or recurrent forms of periodontitis—and may provide additional benefits in case of very deep pockets or specific microbial infections. Systemic antimicrobial therapy is the most effective when the mechanical disruption of the subgingival biofilm is performed during subgingival debridement. The combination of amoxicillin and metronidazole or ciprofloxacin and metronidazole show greater clinical effectiveness than monotherapy $[4,6]$.

The local delivery of antimicrobial drugs may reduce the systemic adverse effects and provide effective drug concentrations in the periodontal pockets to eliminate the pathogens from the subgingival area $[7,8]$. Local application of antibiotics has been advocated for patients with localized lesions or a limited amount of non-responding or recurrent sites [4]. In the literature, many articles are about the development of various types of delivery systems (fibers, films, gels, strips, injectable systems, microparticles) containing different active agents (tetracycline, doxycycline, chlorhexidine, clindamycin, metronidazole, amoxicillin, povidone-iodine) [7,9-22].

In the last 10-15 years, lipid drug delivery received considerable attention due to advancement in the field. Administration of lipid materials is most prevalent in solubility enhancing, lymphatic transport targeting, and/or intestinal transport modulation aiming the increased bioavailability of drug formulations, but creating sustained release systems, covering the bitter or bad taste of active ingredients or protecting drug molecules susceptible to different environmental factors may also be a purpose for the application of lipid excipients [23].

In periodontal therapy, using lipid formulations may provide the prolonged liberation of hydrophilic antimicrobial agents, while protecting them from decomposition in hydrophilic media and masking their unpleasant taste. 
The aim of the recent study was to develop a lipid-based formulation, which can be inserted into the periodontal pocket, has mucoadhesive features and provides sustained delivery of antimicrobial agents, such as amoxicillin, metronidazole and zinc derivatives (zinc hyaluronate, and zinc gluconate). Due to the fact that the applications site possesses special qualities, the following requirements were considered to be met by our drug delivery system: to have a softening point at body temperature to help the adaptation of the dosage form to the shape of periodontal pockets; to be biodegradable to let the delivery system vanish slowly from periodontal pockets as healing commences; to be biocompatible not to trigger further inflammatory processes; and to provide sustained drug release for at least one week. In addition to softening, the purpose of the lipid composition was to create an anhydrous medium, which could protect the antimicrobial agents (amoxicillin) from decomposition and can have a taste-masking effect in the case of metronidazole. This paper also aims to investigate the properties of these delivery systems. During the development, different investigational methods were employed in order to examine the properties of the delivery systems. The measurements carried out included differential scanning calorimetry (DSC), X-ray diffraction (XRD), and mucoadhesion measurements, and also drug diffusion studies and antimicrobial effectiveness investigations.

\section{Materials and Methods}

\subsection{Materials}

Suppocire BP (SBP) pellets (Gattefossé Ltd., Saint-Priest, France), Methocel E4M HPMC (Colorcon Ltd., Dartford, United Kingdom), Kolliphor RH40 (KP) (BASF ChemTrade GmbH, Ludwigshafen, Germany), amoxicillin (AMX) (Antibióticos de León S.L.U., León, Spain), cetostearyl alcohol (CA), metronidazole (MZ), zinc gluconate (ZnGlu) (Ph. Eur. 8., Hungaropharma Plc., Budapest, Hungary), and zinc hyaluronate (ZnHA) (Richter Gedeon Ltd., Budapest, Hungary) were used to create the delivery systems.

For the mucoadhesion and drug dissolution tests, a phosphate-buffered saline (PBS) solution was prepared by dissolving $8 \mathrm{~g} / \mathrm{dm}^{3} \mathrm{NaCl}, 0.2 \mathrm{~g} / \mathrm{dm}^{3} \mathrm{KCl}, 1.44 \mathrm{~g} / \mathrm{dm}^{-3} \mathrm{Na}_{2} \mathrm{HPO}_{4} \cdot 2 \mathrm{H}_{2} \mathrm{O}$ and $0.12 \mathrm{~g} / \mathrm{dm}^{3} \mathrm{KH}_{2} \mathrm{PO}_{4}$ (Ph. Eur. 8., Hungaropharma Plc., Budapest, Hungary) in distilled water. The $\mathrm{pH}$ was adjusted to 7.4 by adding an adequate amount of $0.1 \mathrm{M} \mathrm{HCl}$. The mucin (porcine gastric mucin type II, Sigma-Aldrich, Saint Louis, MO, USA) was used in the form of an $8 w / w \%$ suspension.

\subsection{Composition of the Drug Delivery Systems}

The exact composition of different formulations can be found in Table 1. Five main components were used to create the delivery systems. A lipophilic component SBP was chosen as lipid base and CA was used as a structure-building component. All of the lipid components can be safely used on mucosae, where the SBP can soften at body temperature providing the accommodation of the dosage form to the periodontal pocket.

Table 1. Composition of formulations 1-6. All values are given in $w / w \%$.

\begin{tabular}{ccccccc}
\hline Formulation No. Component & $\mathbf{1}$ & $\mathbf{2}$ & $\mathbf{3}$ & $\mathbf{4}$ & $\mathbf{5}$ & $\mathbf{6}$ \\
\hline Suppocire BP & $33 \%$ & $33 \%$ & $33 \%$ & $47 \%$ & $46.8 \%$ & $32 \%$ \\
\hline Cetostearyl alcohol & $40 \%$ & $40 \%$ & $40 \%$ & $40 \%$ & $40 \%$ & $40 \%$ \\
\hline Kolliphor RH40 & $10 \%$ & $10 \%$ & $10 \%$ & $10 \%$ & $10 \%$ & $10 \%$ \\
\hline Methocel E4M & $2 \%$ & $2 \%$ & $2 \%$ & - & - & - \\
\hline Metronidazole & - & $15 \%$ & $7.5 \%$ & - & - & $15 \%$ \\
\hline Zinc hyaluronate & - & - & - & $3 \%$ & $3 \%$ & $3 \%$ \\
\hline Amoxicillin & $15 \%$ & - & $7.5 \%$ & - & - & - \\
\hline Zinc gluconate & - & - & - & - & $0.2 \%$ & - \\
\hline
\end{tabular}


Cetostearyl alcohol (CA) consists of mainly stearyl and cetyl alcohols; it is a mixture of solid alcohols, it was used to increase the softening temperature of the formulations.

$\mathrm{KP}$, a polyoxyethylene 40 castor oil derivative, was used as a surface-active agent, which helps the wetting of the formulation in the periodontal pocket; thus, it promotes its swelling and erosion.

HPMC (hydroxypropyl methylcellulose) and ZnHA were applied as gelling and mucoadhesive components. During the erosion of the formulations, they can help to prolong the retention time of the systems in the periodontal pockets. HPMC is often used as a mucoadhesive agent in oral formulations such as films [24] or buccal tablets [25] to increase the residence time of the delivery system, while ZnHA is regularly applied in topical gels to accelerate the healing of wounds of different etiologies and to decrease the chance of a possible bacterial superinfection [26], or may be used as a mucoadhesive agent in ophthalmic solutions to lengthen clearance and provide higher efficiency [27].

AMX or MZ, which are regularly applied orally for PD treatment, were incorporated into the vehicles. ZnGlu was also incorporated into one of the formulations as an antimicrobial agent. Zn salts have a known antibacterial effect, which can be useful in periodontitis treatment.

All the formulations were created by a melting and homogenation method. At first, the two lipophilic components (cetostearyl alcohol and Suppocire BP) and the surfactant (Kolliphor RH40) were melted together at $70{ }^{\circ} \mathrm{C}$ on a hot plate (IKA-WERKE RCT B, ETS-D4, IKA Werke GmbH and Co. KG, Staufen im Breisgau, Germany), then the polymer (zinc hyaluronate or Methocel E4M) was suspended and homogenized with an overhead stirrer at 50 RPM (Digital Overhead Stirrer-DLH, VELP Scientifica, Usmate Velate, Italy). The antimicrobial agent(s) (amoxicillin, metronidazole, zinc gluconate) was/were dispersed and homogenized at $50{ }^{\circ} \mathrm{C}$ to avoid thermal decomposition [28]. Delivery systems were created by molding the melted formulations into round shaped silicone molds. Cylindrical drug delivery systems were $1.5 \mathrm{~mm}$ in thickness and $9 \mathrm{~mm}$ in diameter.

\subsection{Differential Scanning Calorimetry (DSC) Measurements}

The softening temperature and the melting point of the formulations were determined by differential scanning calorimetry measurements with a Mettler-Toledo DSC 821e instrument in an argon atmosphere $(100 \mathrm{~mL} / \mathrm{min})$. The temperature was raised from $+5{ }^{\circ} \mathrm{C}$ to $+100{ }^{\circ} \mathrm{C}$ by $5{ }^{\circ} \mathrm{C}$ per minute. Ten milligrams of the samples were put in $40 \mu \mathrm{L}$ aluminum pans. The tops were holed, then the pans were sealed.

\subsection{X-ray Powder Diffraction (XRD) Analysis}

Diffractograms of the raw materials (SBP, CA, KP, MZ, AMX, HPMC, ZnHA, ZnGlu) and the formulations were obtained with a Bruker D8 Advance diffractometer (Bruker AXS GmbH, Billerica, MA, USA) system with $\mathrm{Cu} K \lambda \mathrm{I}$ radiation $(\lambda=1.5406 \AA)$. Each sample was scanned at $40 \mathrm{kV}$ and $40 \mathrm{~mA}$ in the interval of $3^{\circ}-40^{\circ} 2 \theta$, at a scanning speed of $0.1 / \mathrm{s}$ and a step size of $0.010^{\circ}$.

\subsection{Mucoadhesion Measurements}

Adhesion tests were performed with a TA-XT Plus (Texture analyzer, ENCO, Spinea, Italy) instrument equipped with a $1 \mathrm{~kg}$ load cell and a cylinder probe with a diameter of $1.0 \mathrm{~cm}$. Twenty milligrams of the sample attached to the cylinder probe was placed in contact with a filter paper disc impregnated with $50 \mu \mathrm{L}$ mucin dispersion $(8 w / w \%$, prepared in PBS $(\mathrm{pH}=7.4))$. A $2500 \mathrm{mN}$ preload was applied for $3 \mathrm{~min}$ (the compression speed was $2.5 \mathrm{~mm} \mathrm{~min}^{-1}$ ). The cylinder probe was then moved upwards to separate the sample from the substrate at a prefixed speed of $2.5 \mathrm{~mm} \mathrm{~min}{ }^{-1}$. Polymer solutions, prepared by dissolving the solid polymers in PBS, were analyzed during the mucoadhesion measurements where ZnHA and HPMC concentrations were $0.5,1,2,5$, and $10 w / w \%$. Ten parallel measurements were performed. 


\subsection{Investigation of the Swelling and Erosion of Formulations}

A gravimetrical analysis was carried out in order to investigate the water absorption capacity of the different formulations, and to examine the effects of the mucoadhesive polymers on the erosion profile of the delivery systems. Prior to the measurements, the formulations were weighed with an analytical scale and put in $10 \mathrm{~mL}$ of PBS solution thermostated at $37^{\circ} \mathrm{C}$ for various time periods $(0.5,1$, $2,4,6,8,24,36,48,72,96$, and $168 \mathrm{~h}$ ). Subsequent to the designated time period, the formulations were weighed again, which was followed by drying at room temperature. After the formulations had totally dried out, a third weight measurement was carried out. Evaluation of the changes in the mass of the formulations could help the determination of the absorption capacity and the swelling related erosion profiles. For each sampling time three parallels were applied.

\subsection{Drug Dissolution Tests}

The in vitro drug release profiles of formulation nos. 1, 2, 3, and 6 were determined. The compositions were weighed with an analytical scale, put in 50-mm-long dialysis tubes (Spectra/Por ${ }^{\circledR}$ Standard RC tubing, MWCO: $12-14 \mathrm{kD}$ ) and sealed with closures. The tubes containing the formulations were placed in $7.5 \mathrm{~mL}$ of PBS solution thermostated at $37^{\circ} \mathrm{C}$. Drug release was investigated for seven days and three parallel measurements were carried out. One milliliter of samples was taken (at $0.5,1,2,4,6,10,24,30,48,72,96$ and $168 \mathrm{~h}$ ) and were replaced with $1 \mathrm{~mL}$ of PBS solution. The active agents AMX and MZ were separated with an HPLC system (where necessary) and quantified by a UV spectrophotometer attached to the HPLC at $230 \mathrm{~nm}$.

The solution obtained was filtered through a $0.20 \mu \mathrm{m}$ polyethersulfone syringe membrane filter and injected directly into the HPLC system. The AMX and/or MZ content was quantified with a Merck-Hitachi LaChrome Elite HPLC (Hitachi High Technologies America, Inc., Schaumburg, IL, USA). AMX and MZ were measured on a Kinetex $250 \mathrm{~mm} \times 4.6 \mathrm{~mm}$ column packed with $5 \mu \mathrm{m}$ EVO C18, $100 \AA$ (Phenomenex Inc., Torrance, CA, USA). Elution was performed with 20:80 $(v / v)$ Methanol-NaH2PO4 $(0.05 \mathrm{M})$ at a flow rate of $1 \mathrm{~mL} / \mathrm{min}$. Prior to the elution, the eluent was degassed and filtered through a $0.45 \mu \mathrm{m}$ pore-sized glass filter funnel. The run time was seven minutes. Detection was performed via absorption at $230 \pm 4 \mathrm{~nm} .3 \mu \mathrm{L}$ of sample were injected, and the elution was carried out at a sample temperature of $25^{\circ} \mathrm{C}$ and at a column temperature of $25^{\circ} \mathrm{C}$.

Quantitative determination was achieved by comparison with the spectra of standards. The stock solutions of AMX and MZ $(2 \mathrm{mg} / \mathrm{mL})$ were prepared in PBS and stored at $4{ }^{\circ} \mathrm{C}$. The stock solutions were used within $24 \mathrm{~h}$. Working standards $(0.5,1,1.5$, and $2 \mathrm{mg} / \mathrm{mL})$ were prepared freshly by diluting the stock solution with the mobile phase prior to the HPLC analysis. Calibration plots were freshly prepared and were highly linear $\left(R^{2}(\mathrm{AMX})=0.9990\right.$ and $\left.R^{2}(\mathrm{MZ})=0.9998\right)$. Limit of detection (LOD) and limit of quantification (LOQ) of the quantitative determination was 3.308 and $10.020 \mu \mathrm{g} / \mathrm{mL}$, respectively, while in the case of amoxicillin and metronidazol, the resolution $\left(R_{S}\right)$ was 3.10.

Drug recovery tests were also performed, where the incorporated metronidazole was extracted with water at the melting point of the lipid formulations. The results of the extracted drug amounts corresponded to the incorporated drug amounts (99.50 $\pm 0.30 \%)$.

\subsection{Microbiological Investigation}

Microbiological study was conducted in order to measure the antibacterial effectiveness of the developed formulations. Typical periodontopathogenic bacteria: Fusobacterium nucleatum (ATCC ${ }^{\circledR}$ $25586^{\mathrm{TM}}$ ), Parvimonas micra $\left(\right.$ ATCC $^{\circledR} 33270^{\mathrm{TM}}$ ), Eikenella corrodens (ATCC ${ }^{\circledR} 23834^{\mathrm{TM}}$ ), Porphyromonas gingivalis (ATCC ${ }^{\circledR} 33277^{\mathrm{TM}}$ ), Aggregatibacter actinomycetemcomitans (ATCC ${ }^{\circledR} 29524^{\mathrm{TM}}$ ), and Prevotella intermedia (118710) control strains were used. A 1 McFarland standard concentration bacterial suspension of each bacterial strain was made separately with $0.9 \% \mathrm{NaCl}$ solution (in suspension it is equivalent to approximately $3 \times 108$ colony forming units $/ \mathrm{mL}$ ). The suspension was spread onto a horse blood agar plate, where then formulations were placed on. The concentration of the 
active agent(s) was $15 w / w \%: 15 w / w \%$ of $\mathrm{AMX}$ or $\mathrm{MZ}$ alone (formulations 1 and 2 ), or $7.5 w / w \%$ of both AMX and MZ (formulation 3). The delivery systems made with ZnHA contained $3 w / w \%$ $\mathrm{ZnHA}$ (formulation 4) or $3 w / w \% \mathrm{ZnHA}$ and $0.2 w / w \% \mathrm{ZnGlu}$ (formulation 5) or $3 w / w \% \mathrm{ZnHA}$ and $15 w / w \% \mathrm{MZ}$ (formulation 6). After $24 \mathrm{~h}$ of incubation in anaerobic conditions, the diameter of the inhibition zones was measured. The formulations were then put on a new horse blood agar plate, also inoculated with $1 \mathrm{McF}$ arland standard concentration freshly made bacterial suspension of each of the above-mentioned bacterial strains. The plates were then put in an anaerobic chamber for $24 \mathrm{~h}$. This was repeated until no inhibition zone could be detected.

\section{Results and Discussion}

\subsection{Differential Scanning Calorimetry (DSC) Measurements}

DSC was used to determine the softening temperature and the melting point of the formulations. The lipid base of the compositions was SBP, which is often used as a base for suppositories [29]. In our measurements, it has a melting point at $39.08^{\circ} \mathrm{C}$ (Figure 1 ).

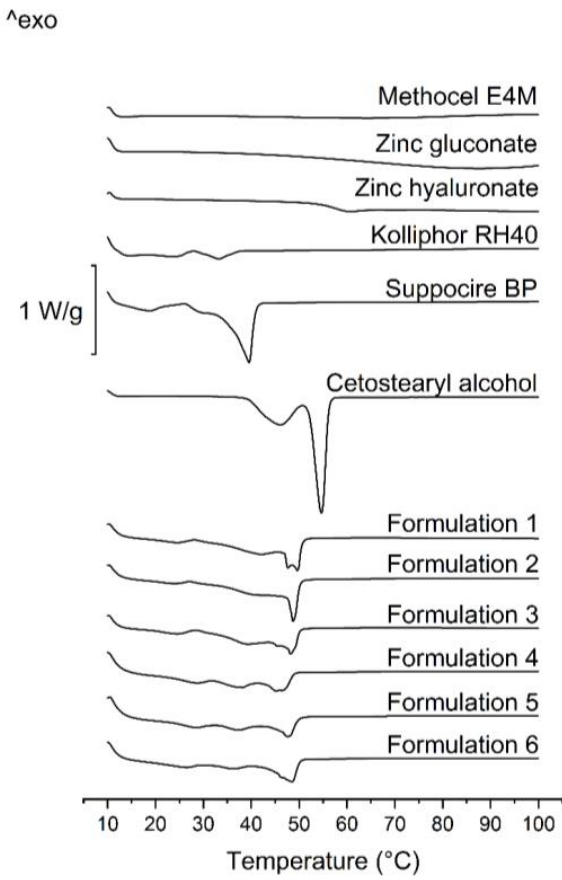

Figure 1. DSC curves of pure components (Kolliphor RH40, Suppocire BP and Cetostearyl alcohol) and formulations containing $15 w / w \%$ of amoxicillin, $15 w / w \%$ of metronidazole, $7.5 w / w \%$ of amoxicillin and metronidazole, $3 w / w \%$ of zinc hyaluronate, $3 w / w \%$ of zinc hyaluronate and $0.2 w / w \%$ of zinc gluconate, and $3 w / w \%$ of zinc hyaluronate and $15 w / w \%$ of metronidazole.

Cetostearyl alcohol is mostly used as a surfactant, a co-surfactant or as a viscosity increasing agent in semisolid formulations. According to our DSC measurements, the melting point of the pure component is at $61{ }^{\circ} \mathrm{C}$ (Figure 1.). In our earlier study [30], we presented that where CA remains partially in a crystalline state; thus, sustained drug release could be achieved.

The results of DSC measurements show that Kolliphor RH40 has a congealing temperature approximately between $20-30{ }^{\circ} \mathrm{C}$ (Figure 1), which corresponds with the literature data [29].

No sharp peaks could be perceived on the curves belonging to the polymers (zinc hyaluronate and Methocel E4M), and zinc gluconate in this temperature range $\left(10-100^{\circ} \mathrm{C}\right)$. Glass transition of these components starts over $50^{\circ} \mathrm{C}$ (Figure 1.)

Formulation nos. 1-6 were examined to investigate the possible modifying effect of the incorporated drugs and excipients on melting point. Antibiotics or other suspended materials may 
partially dissolve in the lipid base, therefore, the effect of various incorporated components of different amounts on the melting point was also investigated.

In all formulations, the sharp peaks of SBP and CA disappeared, even the two peaks of CA morphed into one, and shifted to temperatures between the melting points of the two pure components. At approximately $30^{\circ} \mathrm{C}$, a moderate melting can be observed, but total melting only occurs between 40 and $50{ }^{\circ} \mathrm{C}$. A moderate melting starts at $30^{\circ} \mathrm{C}$, which supports the softening of the systems, but, as there are components with melting points at about $50^{\circ} \mathrm{C}$, which suggests the presence of a coherent structure at body temperature; thus, total melting of the formulations will not occur, and sustained drug release may be possible.

The type of the drug did not influence the melting point greatly, but differences in the shape of the curves are noteworthy. In the case of formulations containing zinc salts or amoxicillin, peaks at approximately $50{ }^{\circ} \mathrm{C}$ (probably the two fused peaks of CA) broadened, meaning an earlier onset of melting, while metronidazole containing ones show sharper peaks.

By the thorough analysis of the DSC results, we could conclude that no peaks of any curve belonging to different formulations could be associated with the decomposition of any component, thus, the constituents are expected to be stable in this temperature range.

\subsection{XRD Analysis}

Active ingredients were suspended in the melted lipids. XRD analyses were carried out to investigate their final state (suspended or dissolved). The presence of solid crystals is indicated by sharp peaks in the diffractograms, while dissolved components, as they do not possess a crystalline structure, are not detectable. The amorphous structure is often a substantial feature of polymers; thus, the crystalline structure and its characteristic peaks are absent from the diffractograms.

All of the components, except for HPMC, Kolliphor RH40, zinc hyaluronate, and zinc gluconate, have a crystalline structure according to the XRD diffractograms presented in Figure 2.

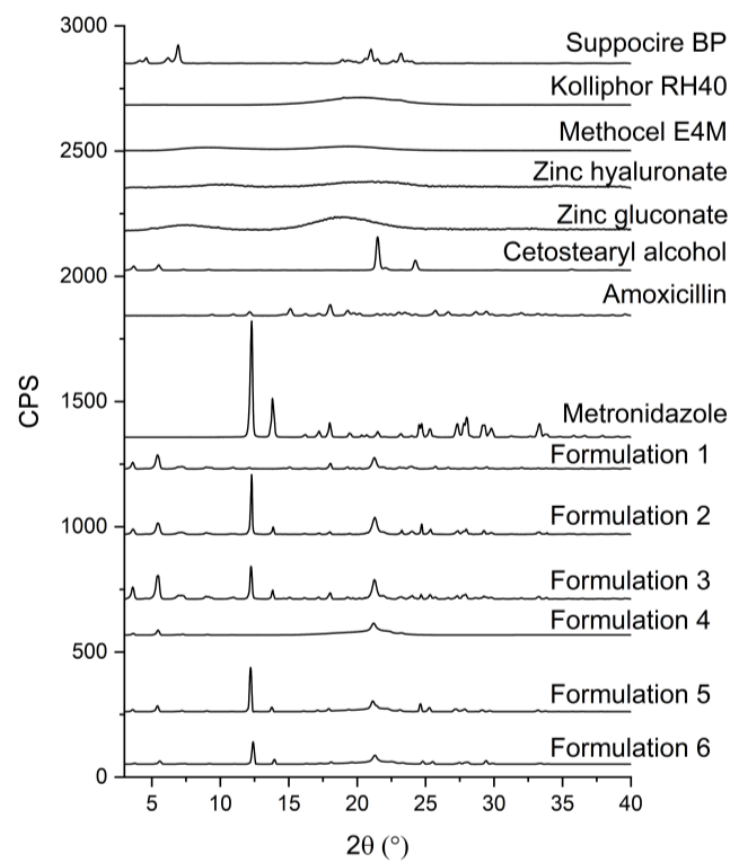

Figure 2. Diffractogram of pure components (Kolliphor RH40, Suppocire BP and cetostearyl alcohol, amoxicillin, metronidazole, zinc hyaluronate, zinc gluconate, Methocel E4M) and formulations containing $15 w / w \%$ of amoxicillin, $15 w / w \%$ of metronidazole, $7.5 w / w \%$ of both of amoxicillin and metronidazole, $3 w / w \%$ of zinc hyaluronate, $3 w / w \%$ of zinc hyaluronate and $0.2 w / w \%$ of zinc gluconate, and $3 w / w \%$ of zinc hyaluronate and $15 w / w \%$ of metronidazole. 
SBP and CA have similar molecular structures, which will result in analogous diffractograms. This means that in the diffractograms of the formulations containing all of the components, it is hard to differentiate between the two lipid components. The more intensive peak of CA could overlap with and cover the peak of SBP at the same $2 \theta$ value in the preparations. This peak, which belongs to CA, has decreased in intensity in the diffractograms of the formulations, which implies that the crystallinity of CA has decreased, which is in accordance with the DSC results, where the two peaks of pure CA have fused together and shifted to a lower temperature range.

A characteristic peak linked to SBP at the $2 \theta$ value of approximately $5^{\circ}$ is present in all formulations with varied intensity, also indicating the crystalline structure of the lipid base.

The characteristic peaks of AMX and MZ could be separated from those of the lipid components. The presence of characteristic peaks in the diffractograms is the sign of solid particles in the formulations. AMX and MZ have a characteristic peak at the $2 \theta$ value of $18.1^{\circ}$ and $12.3^{\circ}$, respectively. Peaks at the mentioned $2 \theta$ values could be observed in the diffractograms of formulations containing AMX or MZ.

The presence of ZnHA or ZnGlu in the preparations could not be demonstrated by this experiment because of their amorphous structure to which no characteristic peak belongs in an XRD diffractogram.

The partially crystalline form of the lipid matrix (CA and SBP) could contribute to the coherent structure of the system, which does not melt at body temperature, thus providing sustained release of antibiotic compounds, while the suspended form of the drugs and antimicrobial agents may provide a more stable form, therefore protecting the drugs from the negative effects of the environment and decomposition as well.

\subsection{Investigation of the Mucoadhesive Properties of the Applied Polymers}

Subsequent to insertion into the periodontal pocket and wetting of the lipid system, the mucoadhesive polymers at the interface of the lipid and the periodontal fluid get hydrated, swell, and form a gel layer, providing adhesion to the application site.

The mucoadhesive properties of the polymers were investigated by means of a tensile test, which is an in vivo-like mucoadhesion method because it mimics the adhesion to a biological surface. Adhesive force and work were determined on the basis of the force-distance curve obtained during the tensile test (Table 2). In this measurement, a larger concentration range of polymers was investigated. The larger concentration range of polymer solutions could mimic the gel layer formed at the surface of the lipid formulation; therefore, the mucoadhesion properties of the delivery system.

Table 2. Adhesive force and work of the gels at different polymer concentrations. Mean values and SD, $n=10$. (ZnHA = Zinc hyaluronate; HPMC = Methocel E4M, hydroxypropyl methylcellulose).

\begin{tabular}{cccccc}
\hline \multirow{2}{*}{ Polymer } & \multirow{2}{*}{ Polymer Conc. (\%) } & \multicolumn{2}{c}{ Adhesive Force $(\mathbf{m N})$} & \multicolumn{2}{c}{ Adhesive Work (mN.mm) } \\
\cline { 2 - 5 } & & \multicolumn{2}{c}{ Mean $\mathbf{\text { SD }}$} & \multicolumn{2}{c}{ Mean \pm SD } \\
\hline \multirow{3}{*}{ ZnHA } & 0.5 & 1579.0 & 71.1 & 167.3 & 9.9 \\
\cline { 2 - 5 } & 1 & 1786.8 & 230.9 & 202.9 & 37.6 \\
\cline { 2 - 5 } & 2 & 1789.6 & 98.9 & 207.1 & 19.6 \\
\cline { 2 - 5 } & 5 & 2299.5 & 111.2 & 397.9 & 30.8 \\
\hline \multirow{3}{*}{ HPMC } & 10 & 2514.4 & 69.6 & 1166.6 & 163.9 \\
\cline { 2 - 5 } & 0.5 & 73.5 & 28.3 & 16.2 & 6.3 \\
\cline { 2 - 5 } & 1 & 145.6 & 62.3 & 28.7 & 17.8 \\
\cline { 2 - 5 } & 2 & 325.7 & 137.1 & 60.7 & 16.1 \\
\hline & 5 & 779.4 & 265.5 & 527.1 & 170.0 \\
\hline
\end{tabular}


In our measurements, in both cases, the adhesive force and work continuously increased with increasing polymer concentrations; ZnHA showed remarkable adhesion over the whole investigated polymer concentration range, indicating better mucoadhesion to the model surface; thus, ZnHA may provide a better mucoadhesive feature for the lipid formulations than HPMC, and the multipotent (adhesive and antimicrobial) characteristics of the former excipient can be beneficial in periodontal therapy.

\subsection{Swelling and Erosion Profiles of Delivery Systems}

In addition to melting and softening, swelling and erosion of the lipid formulation can also affect drug release. In this case, the swelling of the polymer can result in a gel layer on the surface of the delivery system, and then this layer can be slowly removed thanks to the elimination mechanisms in the oral cavity and periodontal pocket. In addition to this mechanism, the melted lipid and the surfactant can also be eliminated from the formulation by emulsification with the produced oral biological medium, the gingival crevicular fluid.

In our experiments the swelling ratio (\%) was calculated as follows [31]:

$$
\text { Swelling ratio }(\%)=m_{\mathrm{s}} / m_{\mathrm{o}} \times 100 \%
$$

where $m_{\mathrm{s}}$ is the mass of the formulations after immersed in water for the specified time and $m_{\mathrm{o}}$ is the mass of the original formulations (prior to the immersion into water).

During the measurements, delivery systems were immersed into purified water to investigate the swelling and erosion properties of the different preparations, because different antimicrobial agents and mucoadhesive polymers are incorporated into the systems. The swelling ratio of the different compositions can be observed in Figure 3a,b, while the erosion profiles are shown in Figure 4a,b.
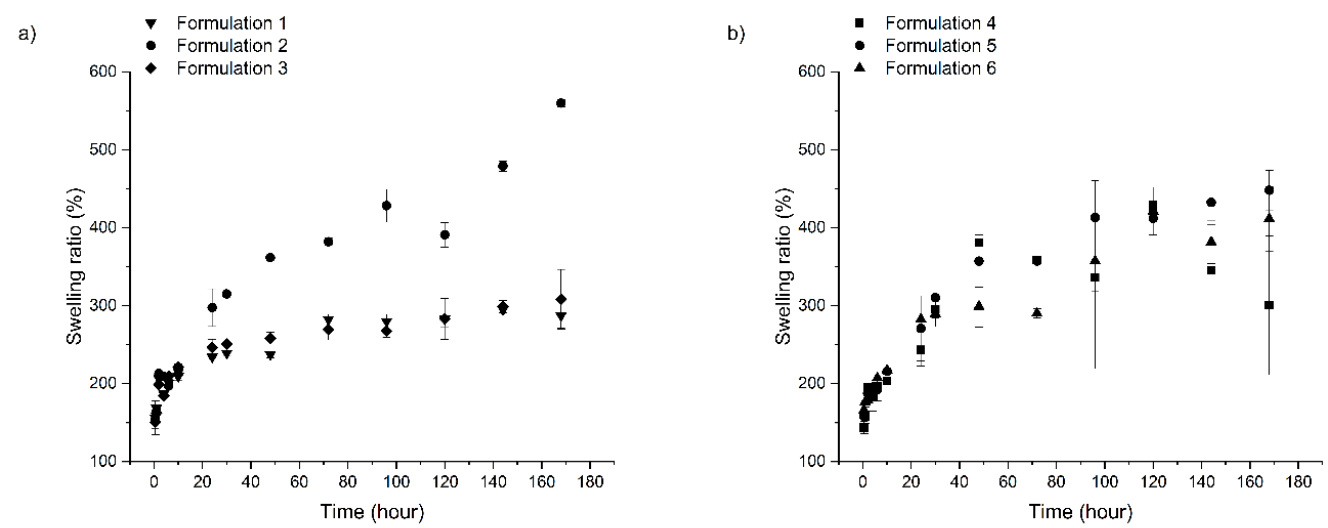

Figure 3. Swelling profiles of formulations: (a) $1-3$ and (b) $4-6$.
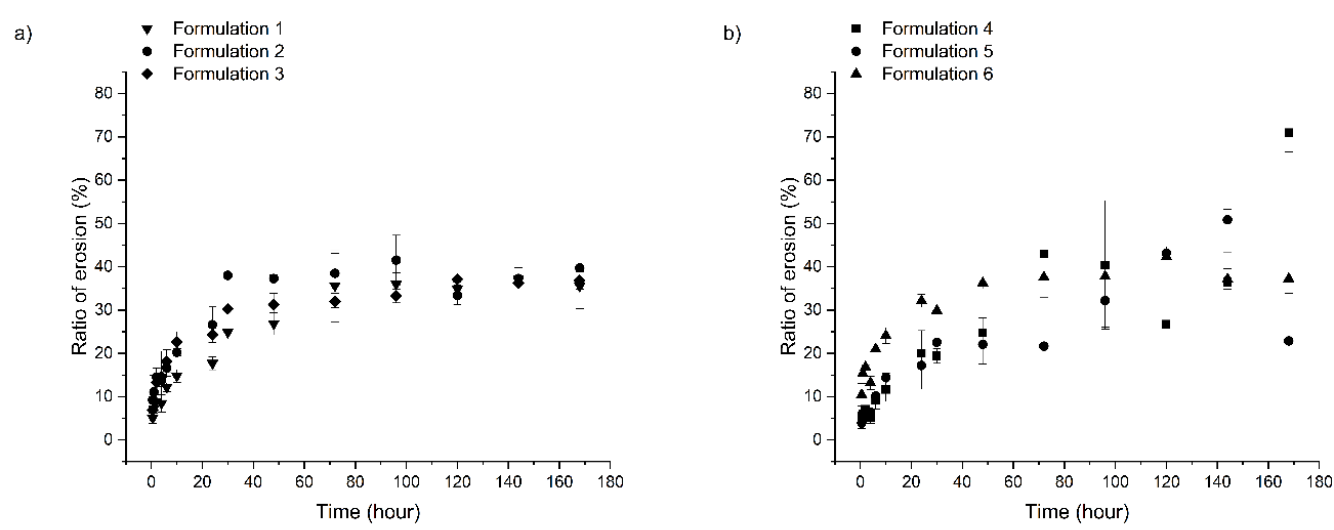

Figure 4. Erosion of formulations: (a) 1-3 and (b) 4-6. 
Based on the swelling profile, two groups can be distinguished. Swelling ratio of formulations containing ZnHA and/or a higher amount of MZ shows a higher water absorption capacity than formulations with AMX or lower concentrations of MZ and HPMC as polymer; the former group had a maximum swelling ratio of approximately $560 \%$, while the latter group was only approximately $300 \%$.

This phenomenon may be driven by the different swelling and solubility of components: i) ZnHA is a material possessing a strong water absorption capacity, and ii) MZ owns a sparingly water-soluble feature but better than amoxicillin. Because of the higher water absorption-capacity of ZnHA and better solubility of MZ, water could access the lipid structure with ease resulting in higher swelling of formulations; ZnHA may bind a very large amount of water and break the coherent lipid structure, and through the generated superficial microgaps more ZnHA particles become accessible to water, thus binding more medium. MZ may work in a highly similar way, but in this case the applied polymer (HPMC) binds the water. The better solubility of MZ compared to AMX, also contributes to quicker dissolution from the systems, and the dissolved MZ (leaving capillaries in the structure) may provide water with a trouble-free entry accessing polymer particles incorporated into the delivery systems. The more drug is dissolved from the preparations, the more capillaries are made, and through more capillaries, more polymer particles may be accessed, which results in a higher water binding. When ZnHA and a high concentration of $\mathrm{MZ}(15 w / w \%)$ are applied together, no extra swelling can be observed. This phenomenon can be explained by the structure of the formulations, the swelling power of the polymers may not be vigorous enough to destroy the coherent solid structure of the delivery system; thus, combining the two components ( $\mathrm{MZ}$ and $\mathrm{ZnHA}$ ) did not result in over-swelling.

AMX and lower concentrations of MZ are not capable of generating the same effect due to the creation of fewer capillaries because of the poorer solubility of amoxicillin compared to $\mathrm{MZ}$ and the lower concentration of MZ; therefore, the swelling of those systems remains below the level provided by the formulations having $\mathrm{ZnHA}$ or more $\mathrm{MZ}$ incorporated into them.

Accordingly, the swelling profiles of the different formulations may be dependent on the incorporated materials, their attributes and the strength of the coherent lipid structure.

Erosion ratio (\%) was calculated from the following equation [30]:

$$
\text { Erosion ratio }(\%)=m_{\mathrm{o}}-m_{\mathrm{d}} / m_{\mathrm{o}} \times 100 \%
$$

where $m_{\mathrm{o}}$ is the mass of the original formulations and $\mathrm{m}_{\mathrm{d}}$ is the mass of the dried formulation after the swelling and erosion measurements.

In contrast with the results of the swelling measurements, the erosion of different compositions are not highly different from each other (Figure $4 a, b)$. The weight of all formulations has decreased to approximately $60 \%$ of that of the original ones. Two main mechanisms may control the erosion of the drug delivery systems. One is the emulsification of components melted at body temperature $\left(37^{\circ} \mathrm{C}\right)$ with water. The other is the swelling power of incorporated polymers, destroying the coherent structure, and the erosion of the gel layer formed at the surface. The similarities in the erosion profiles can highlight that the destruction of delivery systems may be more likely to be defined by the emulsification of the melted lipid components, as the lipid composition and ratio and, therefore, the basic structure of the different compositions, are almost the same. The swelling experiments also suggest the same findings, because the strength of the coherent structure could limit the swelling of polymers.

\subsection{Investigation of Drug Release from Formulations}

Drug dissolution measurements were carried out in order to evaluate the drug release profiles of the prepared formulations which contain MZ. An HPLC method was used to quantify the dissolved drug from the preparations.

The amount of released MZ from formulations containing the antibiotic drug is shown in Figure 5. Preparations containing $15 w / w \%$ MZ and HPMC or ZnHA have shown similar drug release profiles. 
A plateau phase commencing at approximately at the 100th hour is indicated in both cases, which is in accordance with a total drug release.

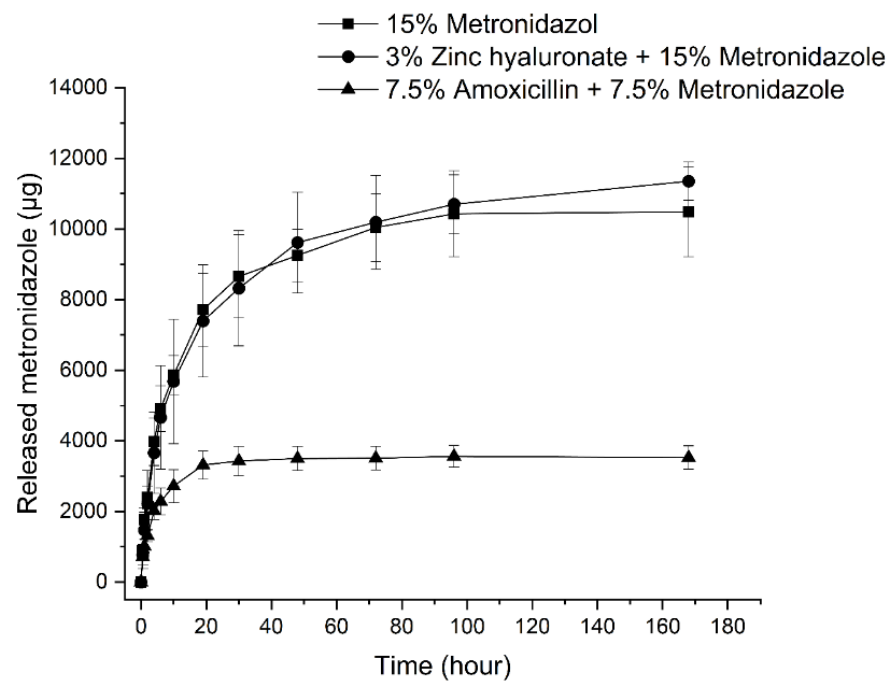

Figure 5. Released amount of metronidazole $(\mu \mathrm{g})$ from formulations with different compositions (15 $w / w \%$ metronidazole, $3 w / w \%$ zinc hyaluronate and $15 w / w \%$ metronidazole, and $7.5 w / w \%$ amoxicillin and $7.5 w / w \%$ metronidazole).

However, the drug release curves of formulations containing $7.5 w / w \% \mathrm{MZ}$ indicates that only one-third of the amount was liberated from the preparations despite the fact that half of the amount had been incorporated into the compositions.

The drug release mechanism can be characterized with the following exponential equation:

$$
M_{\mathrm{t}} / M_{\infty}=k \times t^{n}
$$

where $M_{t} / M_{\infty}$ is the fraction of drug released, $k$ is the kinetic constant and $n$ is the release exponent describing the mechanism of the release.

On the basis of literature data, in case of swelling controlled cylindric drug delivery systems, if the release exponent is 0.45 , the dug release mechanism is a Fickian diffusion, while the release exponent is between 0.45 and 0.89 , the release corresponds to anomalous (non-Fickian) diffusion [32]. In our case, the highly swellable formulations (Formulations 2 and 6) showed non-Fickian diffusion, which indicates that Fickian diffusion coupled with the swelling of the incorporated polymer. In the formulation where swelling is moderated (Formulation 3) alone Fickian diffusion characterizes the drug release $(n=0.4567)$ (Table 3).

Table 3. Release exponent $(n)$, kinetic constant $(k)$ and $R^{2}$ values of curves fitted on the drug release curves of formulations 2,3 , and 6 .

\begin{tabular}{cccc}
\hline Formulation No. & $\boldsymbol{n}$ & $\boldsymbol{k}$ & $\boldsymbol{R}^{\mathbf{2}}$ \\
\hline 2 & 0.6237 & 1555 & 0.9802 \\
\hline 3 & 0.4567 & 995.6 & 0.9926 \\
\hline 6 & 0.6671 & 1360.6 & 0.9880 \\
\hline
\end{tabular}

The phenomenon detailed above in Section 3.4. may be in the background of such results. MZ, which is a more soluble material in water compared to AMX, could be liberated more quickly from formulations creating capillaries, which contribute to a higher swelling and therefore allow water to access all the suspended drug in the delivery systems. On the contrary, when smaller amounts of MZ 
are incorporated into the delivery systems with AMX - a substance with lower water solubility-fewer capillaries may be formed during drug release, permitting less water to penetrate the systems and resulting in non-complete drug dissolution.

The application of $\mathrm{ZnHA}$ did not change the release profile, which can be explained by the similar swelling and erosion profiles of the formulation containing $15 w / w \%$ MZ with and without ZnHA.

Summarizing the results of drug dissolution testing, a sustained release of drugs could be achieved with these compositions, and the main factors affecting drug release are swelling (driven by the applied hydrophilic components such as polymer, active ingredients and their concentration) and the strength of the coherent lipid structure.

\subsection{Microbiological Investigation}

Numerous different species of microorganisms may be present in the oral cavity or on the dental surfaces. The composition of the oral microbiota varies widely from person to person, from place to place and naturally with dietary habits [33,34].

Many journal articles aim to define the species of bacteria which have a contribution to the initiation and progression of periodontal disease [33-41], but the high number of different strains and cultivation difficulties make it almost impossible. Moreover, only a small percentage of the subgingival oral microbiota has a role in disease pathogenesis [42,43]. Pathogen organisms which could be linked to and may have correspondence to the disease are the following: Porphyromonas gingivalis, Prevotella intermedia, Bacteroides forsythus, Actinobacillus actinomycetemcomitans, Treponema denticola, Tannerella forsythia, Fusobacterium nucleatum, Fusobacterium periodonticum, Prevotella intermedia, Prevotella nigrescens, Parvimonas micra, Campylobacter gracilis, Campylobacter rectus, Campylobacter showae, Eubacterium nodatum, Streptococcus constellatus, Eikenella corrodens, Streptococcus spp., etc. [33,42-45]. According to Kolenbrander et. al. [45], these species can be separated into two categories concerning plaque formation. Species in the first category-initial colonizers-are thought to stick to the tooth surface and proliferate. The second group-late colonizers-binds to the first group of bacteria via different interactions.

In our formulations, $\mathrm{AMX}$ or $\mathrm{MZ}$ was incorporated in $15 w / w \%$ concentration, and a system containing 7.5/7.5 w/w\% AMX and MZ was also compiled. In addition to these formulations, ZnHA, $\mathrm{ZnHA}$ and ZnGlu, and ZnHA and MZ containing ones were also created and investigated.

AMX, which is a semi-synthetic penicillin derivative, is known to be effective against many aerobic or anaerobic Gram-positive and Gram-negative bacteria [13,46].

$\mathrm{MZ}$ is an antibacterial agent, included in the nitroimidazole group. Numerous bacteria and protozoa - even anaerobes-are known to be susceptible to MZ [7,47].

In periodontal disease therapy, MZ and AMX may be used as an adjunct to scaling and root planning therapies locally or systematically [48-50].

Hyaluronic acid is a naturally occurring polysaccharide of the extracellular matrix of connective tissue, synovial fluid, and soft periodontal tissues as well. Its application in the treatment of the inflammatory process is established in different medical areas such as orthopedics, dermatology, and ophthalmology. In the treatment of periodontal diseases, hyaluronic acid shows anti-inflammatory and anti-bacterial effects [51].

Zinc salts may have a beneficial effect when used in mouth rinses, can possibly decrease plaque formation by inhibiting glycolytic enzymes and may prevent the attachment of bacteria to the tooth surface [52]. These salts administered in combination with other antimicrobial agents may show a synergism by means of antibacterial effect [53].

In view of the beneficial properties of hyaluronates, zinc hyaluronan was chosen as a combined mucoadhesive and antimicrobial agent for our other set of formulations.

The antibacterial effectiveness of samples with Zinc hyaluronate was also measured to determine if the polymer has an antibiotic effect alone or in combination with other molecules like zinc gluconate or metronidazole. 
In our microbiological investigation, where the antimicrobial activity of our formulations was measured, we used six different strains of oral pathogen bacteria, which may contribute to the initiation of periodontitis. These were the following: Eikenella corrodens, Prevotella intermedia, Parvimonas micra, Fusobacterium nucleatum, Aggregatibacter actinomycetemcomitans, and Porphyromonas gingivalis.

Eikenella corrodens - a facultative anaerobic, Gram-negative bacterium-is a human (mostly oral) pathogen. E. corrodens appears to be susceptible to beta-lactam antibiotics, but resistant to metronidazole [54].

Parvimonas micra (also known as Peptostreptococcus micros or Micromonas micros) —an anaerobic, Gram-positive coccus-is the member of the normal human gastrointestinal flora [55]. According to Rams et al., it shows high susceptibility to penicillin but metronidazole seems to be less effective against the bacterium [56].

Prevotella intermedia-a Gram-negative anaerobic bacterium with black pigments-is often connected with oral and subgingival diseases. P. intermedia shows susceptibility to penicillin and metronidazole [57].

Fusobacterium nucleatum - a mostly oral and periodontal anaerobic pathogen—can be linked to a variety of human diseases. Strains isolated from endodontic infections seem to be highly sensitive to amoxicillin and a little less susceptible to metronidazole [58,59].

Aggregatibacter actinomycetemcomitans-a Gram-negative, facultatively anaerobic rod-is frequently associated with most forms of periodontitis and numerous oral infections. Amoxicillin is effective against the bacterium, while it shows much less susceptibility to metronidazole $[60,61]$.

Porphyromonas gingivalis - an obligately anaerobic, Gram-negative black-pigmented rod-is the most commonly linked microorganism to periodontal disease. According to susceptibility tests, $P$. gingivalis is sensitive to amoxicillin and metronidazole $[62,63]$.

Agar plates were $90.0 \mathrm{~mm}$ in diameter, thus, the largest possibly detectable inhibition zone could be around $90.0 \mathrm{~mm}$.

The results of the microbiological investigations can be found in Figure 6.

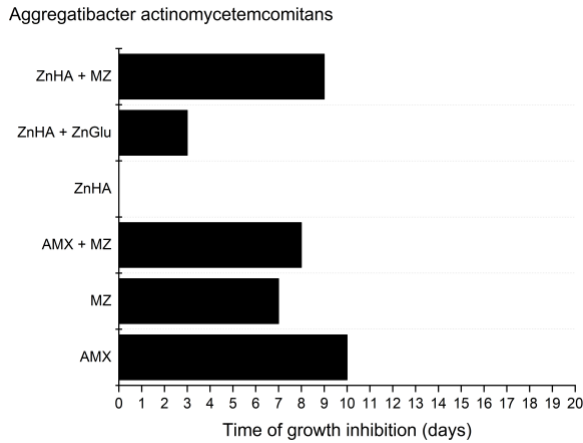

(a)

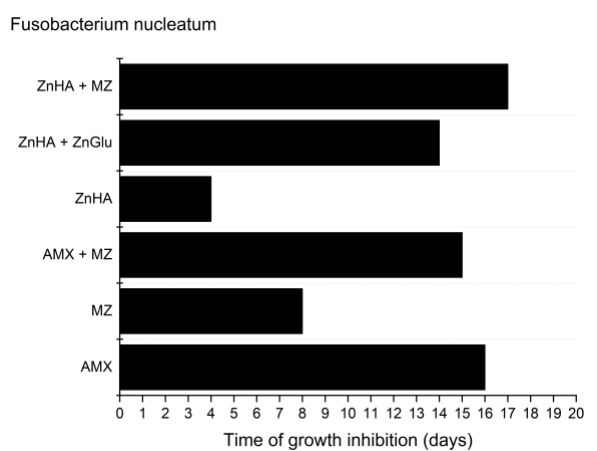

(c)

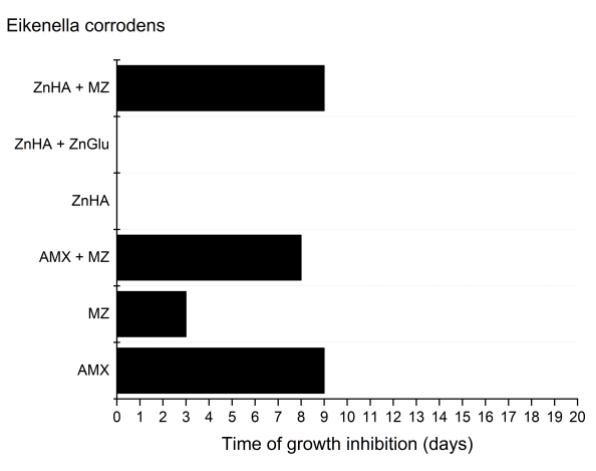

(b)

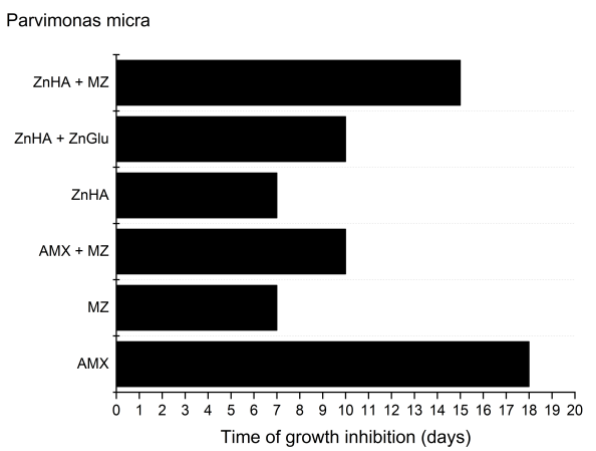

(d)

Figure 6. Cont. 


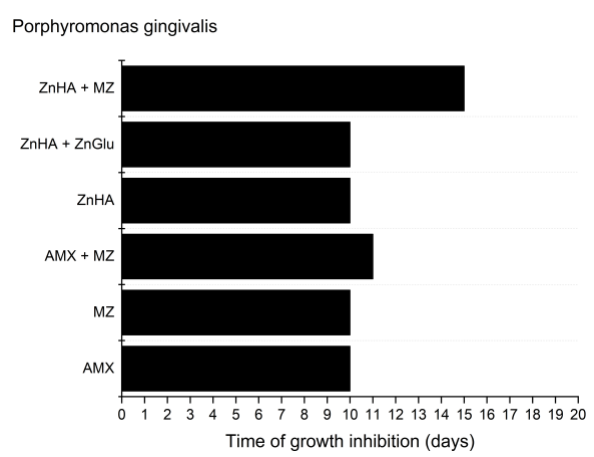

(e)

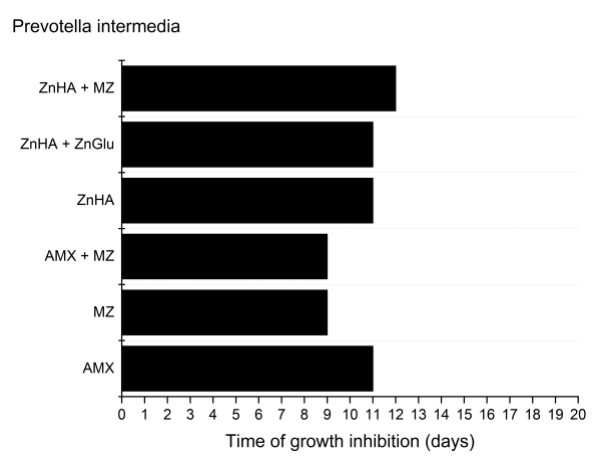

(f)

Figure 6. Effectiveness of delivery systems containing different antimicrobial agents against various oral anaerobic pathogenic bacteria: (a) Aggregatibacter actinomycetemcomitans; (b) Eikenella corrodens; (c) Fusobacterium nucleatum; (d) Parvimonas micra; (e) Porphyromonas gingivalis; and (f) Prevotella intermedia.

The largest measurable inhibition zone was $74.0 \mathrm{~mm}$ in diameter and was because of an amoxicillin containing formulation on an agar plate inoculated with Eikenella corrodens after the first $24 \mathrm{~h}$.

According to our measurements, Parvimonas micra was the most sensitive microorganism to AMX containing formulations as the compositions could provide 17 days of effective drug release, while the least susceptible pathogen was Eikenella corrodens with only eight days of growth inhibition.

MZ susceptibility was slightly lower, as the effect against the most sensitive bacterium (Porphyromonas gingivalis) was only nine days. Eikenella corrodens was the least susceptible to MZ as on the first 2 days no growth could be detected, but after two days bacteria were growing all over the agar plate, and there was no inhibition zone around the formulation. This result is in accordance with the literature data, where it was established that Eikenella corrodens is resistant to MZ [46].

Compositions containing $7.5 w / w \%$ of AMX and MZ did not provide larger inhibition zones than formulations containing only one antimicrobial agent as the antimicrobial effect lasted for shorter time periods. P. gingivalis was an exception: this bacterial strain was the only one with higher susceptibility to the combination of the two antimicrobial drugs. In most cases, the growth inhibition effect of combinations lasted longer than that of metronidazole. This could have been possible due to the more potent antimicrobial effect of AMX. Lower susceptibility to the combination of AMX and MZ compared to only AMX containing formulations may be due to the decreased concentration of AMX in the combination containing compositions and the lower susceptibility of bacteria to MZ.

Whereas MZ is less potent against Aggregatibacter actinomycetemcomitans, Fusobacterium nucleatum, and Parvimonas micra [56,58-61], with our formulations the growth inhibition effect can be provided for up to 3 days.

Susceptibility to ZnHA is different among various bacterial strains. Aggregatibacter actinomycetemcomitans and Eikenella corrodens show resistance to ZnHA, but combined with ZnGlu, a longer effect can be observed in case of Aggregatibacter actinomycetemcomitans. Eikenella corrodens remains unsusceptible to the combination of ZnHA and ZnGlu.

According to the results, the same susceptibility characterizes Porphyromonas gingivalis and Prevotella intermedia when using ZnHA alone or in combination with ZnGlu.

In case of Parvimonas micra and Fusobacterium nucleatum, the results show that higher efficiency may be achieved by administering a combination of ZnHA and ZnGlu instead of using only ZnHA.

The results imply that the combination of $\mathrm{MZ}$ and ZnHA provides a synergistic effect: formulations containing both ZnHA and $\mathrm{MZ}$ provided longer growth inhibition against the microorganisms than the active agents alone. The synergistic effect is most conspicuous against Eikenella corrodens where formulations with $\mathrm{MZ}$ only lasted for two days, and ZnHA had no antibacterial effect against the bacteria, while the combination showed a nine-day effect. 
All in all, the outcome of the antimicrobial investigation of ZnHA supports that alone it may not be effective enough, but a combination with an antimicrobial drug (e.g., MZ) may provide a synergistic and-in this case-a longer positive effect.

While the in vitro dissolution of AMX could not be detected, because the antibiotic had decomposed thanks to the test environment (aqueous medium at $37{ }^{\circ} \mathrm{C}$ ), the results of the microbiological investigation imply that AMX stays stable when incorporated into the lipid preparations, as it may provide 18 days of growth inhibition. (In this experiment, anaerobic environment was provided, and the agar plates were changed day-by-day, which enabled amoxicillin to remain stable in the aqueous agar medium.) This finding and the swelling experiments suggest that the combination of AMX and a highly hydrophilic polymer with strong water binding capacity may not be beneficial, because the sustained release of AMX could easily result in the decomposition of the drug, even in the formulation prior to its dissolution.

\section{Conclusions}

An anhydrous lipid-based drug delivery system for the treatment of periodontitis was successfully prepared and investigated. Possible administration and positive effect during disease treatment were confirmed by the results of different measurements.

According to the DSC measurements, the melting of formulations starts at body temperature, but total melting only occurs over $40{ }^{\circ} \mathrm{C}$, which allows the presence of a coherent structure and provides sustained drug release.

X-ray diffraction analysis indicates that the antibiotics are in a suspended form in the compositions, and lipid components have a crystalline structure, which may also contribute to a prolonged drug release and antimicrobial effect.

Thanks to the complex composition, our lipid formulations are swellable and degradable systems. On the basis of our results, their swelling can be driven by the applied hydrophilic components (polymer, active ingredients), their concentration and the strength of the coherent lipid structure, while their erosion may be controlled mainly by the emulsification of the melted lipid components.

In formulations, where swelling is remarkable, Fickian diffusion is coupled with the swelling of the incorporated polymer. Because of this phenomenon, which is indicated in the shape of the diffusion curves, an anomalous (non-Fickian) diffusion model could be fitted to the diffusion curves. The results of drug release and antimicrobial investigations show that with AMX incorporated into the delivery systems, long drug diffusion can be obtained with a high antimicrobial effect. AMX can be protected against degradation in hydrophilic circumstances at the periodontal pocket by using these lipid formulations, which is suggested by our antimicrobial investigations. The sustained drug release of $\mathrm{MZ}$ can also be provided, which may enable taste-masking and direct application of MZ in the oral cavity without decreasing patient compliance. The combination of ZnHA and MZ improved the antimicrobial characteristics of formulations compared to formulations containing only ZnHA or MZ. These findings suggest that-when administered together-the two compounds have a synergistic effect and might be a good alternative for patients allergic to penicillin and their derivatives.

In order to improve the residence time of melted lipid formulations in the periodontal pocket, mucoadhesive polymers were applied. In our experiments, ZnHA had better adhesivity compared to HPMC, and, additionally, it has a potential antibiotic feature. Nevertheless, the application of a highly hydrophilic polymer with a strong water absorption capacity is questionable when the drug, e.g., AMX, is not stable in aqueous circumstances.

The developed and evaluated lipid formulations containing antimicrobials may offer a solution to such problems of local periodontal therapy as long-lasting effect. Moreover, the combination of MZ and ZnHA can provide an effective antimicrobial therapy for penicillin-allergic patients.

Author Contributions: Investigation: A.L., E.U., and A.G.; writing-original draft: A.L. and M.B.-S.; writing-review and editing: P.V., S.B., A.K., and E.C. 
Funding: This research received no external funding.

Acknowledgments: We would like to express our gratitude to Azelis Hungary Ltd. and BASF Hungary Ltd. for providing us with Suppocire BP pellets and Kolliphor RH40.

Conflicts of Interest: The authors declare no conflict of interest.

\section{References}

1. Genco, R.J.; Borgnakke, W.S. Risk factors for periodontal disease. Periodontology 2000 2013, 62, 59-94. [CrossRef] [PubMed]

2. Tonetti, M.S.; Jepsen, S.; Jin, L.; Otomo-Corgel, J. Impact of the global burden of periodontal diseases on health, nutrition and wellbeing of mankind: A call for global action. J. Clin. Periodontol. 2017, 44, 456-462. [CrossRef]

3. Chapple, I.L.C. Time to take periodontitis seriously. BMJ 2014, 348, g2645. [CrossRef]

4. Graziani, F.; Karapetsa, D.; Alonso, B.; Herrera, D. Nonsurgical and surgical treatment of periodontitis: How many options for one disease? Periodontology 2000 2017, 75, 152-188. [CrossRef]

5. Tomasi, C.; Koutouzis, T.; Wennström, J.L. Locally Delivered Doxycycline as an Adjunct to Mechanical Debridement at Retreatment of Periodontal Pockets. J. Periodontol. 2008, 79, 431-439. [CrossRef] [PubMed]

6. Sanz, M.; Teughels, W. Innovations in non-surgical periodontal therapy: Consensus Report of the Sixth European Workshop on Periodontology. J. Clin. Periodontol. 2008, 35, 3-7. [CrossRef]

7. Schwach-Abdellaoui, K. Local delivery of antimicrobial agents for the treatment of periodontal diseases. Eur. J. Pharm. Biopharm. 2000, 50, 83-99. [CrossRef]

8. Bollen, C.M.L.; Quirynen, M. Microbiological Response to Mechanical Treatment in Combination with Adjunctive Therapy. A Review of the Literature. J. Periodontol. 1996, 67, 1143-1158. [CrossRef]

9. Do, M.P.; Neut, C.; Metz, H.; Delcourt, E.; Mäder, K.; Siepmann, J.; Siepmann, F. In-situ forming composite implants for periodontitis treatment: How the formulation determines system performance. Int. J. Pharm. 2015, 486, 38-51. [CrossRef]

10. Agarwal, R.K.; Robinson, D.H.; Maze, G.I.; Reinhardt, R.A. Development and characterization of tetracycline-poly(lactide/glycolide) films for the treatment of periodontitis. J. Control. Release 1993, 23, 137-146. [CrossRef]

11. Sundararaj, S.C.; Thomas, M.V.; Dziubla, T.D.; Puleo, D.A. Bioerodible system for sequential release of multiple drugs. Acta Biomater. 2014, 10, 115-125. [CrossRef] [PubMed]

12. Bromberg, L.E.; Braman, V.M.; Rothstein, D.M.; Spacciapoli, P.; O'Connor, S.M.; Nelson, E.J.; Buxton, D.K.; Tonetti, M.S.; Friden, P.M. Sustained release of silver from periodontal wafers for treatment of periodontitis. J. Control. Release 2000, 68, 63-72. [CrossRef]

13. Jain, N.; Jain, G.K.; Javed, S.; Iqbal, Z.; Talegaonkar, S.; Ahmad, F.J.; Khar, R.K. Recent approaches for the treatment of periodontitis. Drug Discov. Today 2008, 13, 932-943. [CrossRef]

14. Al-Saeed, M.Y.; Babay, N. The use of povidone-iodine and hydrogen peroxide mixture as an adjunct to non-surgical treatment of slight to moderate chronic periodontitis. Saudi Dent. J. 2009, 21, 127-133. [CrossRef]

15. Do, M.P.; Neut, C.; Metz, H.; Delcourt, E.; Siepmann, J.; Mäder, K.; Siepmann, F. Mechanistic analysis of PLGA/HPMC-based in-situ forming implants for periodontitis treatment. Eur. J. Pharm. Biopharm. 2015, 94, 273-283. [CrossRef]

16. Tyagi, P.; Vaish, S.; Dodwad, V. Clinical efficacy of subgingivally delivered $0.5 \%$ controlled release azithromycin gel in the management of chronic periodontitis. Indian J. Med. Sci. 2011, 65, 223-230.

17. Do, M.P.; Neut, C.; Delcourt, E.; Seixas Certo, T.; Siepmann, J.; Siepmann, F. In situ forming implants for periodontitis treatment with improved adhesive properties. Eur. J. Pharm. Biopharm. 2014, 88, 342-350. [CrossRef] [PubMed]

18. Kopytynska-Kasperczyk, A.; Dobrzynski, P.; Pastusiak, M.; Jarzabek, B.; Prochwicz, W. Local delivery system of doxycycline hyclate based on $\epsilon$-caprolactone copolymers for periodontitis treatment. Int. J. Pharm. 2015, 491, 335-344. [CrossRef] [PubMed]

19. Chinta, D.P.; Katakam, P.; Murthy, V.S.N.; Newton, M.J. Formulation and in-vitro evaluation of moxifloxacin loaded crosslinked chitosan films for the treatment of periodontitis. J. Pharm. Res. 2013, 7, 483-490. [CrossRef] 
20. Mayol, L.; Quaglia, F.; Borzacchiello, A.; Ambrosio, L.; Rotonda, M.I. La A novel poloxamers/hyaluronic acid in situ forming hydrogel for drug delivery: Rheological, mucoadhesive and in vitro release properties. Eur. J. Pharm. Biopharm. 2008, 70, 199-206. [CrossRef]

21. Lee, B.-S.; Lee, C.-C.; Lin, H.-P.; Shih, W.-A.; Hsieh, W.-L.; Lai, C.-H.; Takeuchi, Y.; Chen, Y.-W. A functional chitosan membrane with grafted epigallocatechin-3-gallate and lovastatin enhances periodontal tissue regeneration in dogs. Carbohydr. Polym. 2016, 151, 790-802. [CrossRef] [PubMed]

22. Sundararaj, S.C.; Thomas, M.V.; Peyyala, R.; Dziubla, T.D.; Puleo, D.A. Design of a multiple drug delivery system directed at periodontitis. Biomaterials 2013, 34, 8835-8842. [CrossRef] [PubMed]

23. Jannin, V.; Musakhanian, J.; Marchaud, D. Approaches for the development of solid and semi-solid lipid-based formulations. Adv. Drug Deliv. Rev. 2008, 60, 734-746. [CrossRef]

24. Perioli, L.; Ambrogi, V.; Angelici, F.; Ricci, M.; Giovagnoli, S.; Capuccella, M.; Rossi, C. Development of mucoadhesive patches for buccal administration of ibuprofen. J. Control. Release 2004, 99, 73-82. [CrossRef]

25. Ramesha Chary, R.B.; Vani, G.; Rao, Y.M. In Vitro and In Vivo Adhesion Testing of Mucoadhesive Drug Delivery Systems. Drug Dev. Ind. Pharm. 1999, 25, 685-690. [CrossRef] [PubMed]

26. Juhász, I.; Zoltán, P.; Erdei, I. Treatment of partial thickness burns with Zn-hyaluronan: Lessons of a clinical pilot study. Ann. Burns Fire Disasters 2012, 25, 82-85.

27. Horvát, G.; Budai-Szucs, M.; Berkó, S.; Szabó-Révész, P.; Soós, J.; Facskó, A.; Maroda, M.; Mori, M.; Sandri, G.; Bonferoni, M.C.; et al. Comparative study of nanosized cross-linked sodium-, linear sodium- and zinc-hyaluronate as potential ocular mucoadhesive drug delivery systems. Int. J. Pharm. 2015, 494, 321-328. [CrossRef]

28. Svahn, O.; Björklund, E. Thermal stability assessment of antibiotics in moderate temperature and subcritical water using a pressurized dynamic flow-through system. Int. J. Innov. Appl. Stud. 2015, 11, 2028-9324.

29. Rowe, R.C.; Sheskey, P.J.; Owen, S.C. Handbook of Pharmaceutical Excipients, 6th ed.; Pharmaceutical Press: London, UK, 2009; ISBN 978-0-85369-792-3.

30. Leber, A.; Budai-Szucs, M.; Urban, E.; Valyi, P.; Kovacs, A.; Berko, S.; Csanyi, E. Formulation and Investigation of a Lipid Based Delivery System Containing Antimicrobials for the Treatment of Periodontal Disease. Curr. Drug Deliv. 2018, 15, 887-897. [CrossRef] [PubMed]

31. Horvát, G.; Gyarmati, B.; Berkó, S.; Szabó-Révész, P.; Szilágyi, B.Á.; Szilágyi, A.; Soós, J.; Sandri, G.; Bonferoni, M.C.; Rossi, S.; et al. Thiolated poly(aspartic acid) as potential in situ gelling, ocular mucoadhesive drug delivery system. Eur. J. Pharm. Sci. 2015, 67, 1-11. [CrossRef]

32. Ritger, P.L.; Peppas, N.A. A simple equation for description of solute release II. Fickian and anomalous release from swellable devices. J. Control. Release 1987, 5, 37-42. [CrossRef]

33. Moore, W.E.C.; Moore, L.V.H. The bacteria of periodontal diseases. Periodontology 2000 1994, 5, 66-77. [CrossRef]

34. Costalonga, M.; Herzberg, M.C. The oral microbiome and the immunobiology of periodontal disease and caries. Immunol. Lett. 2014, 162, 22-38. [CrossRef] [PubMed]

35. Darby, I.; Curtis, M. Microbiology of periodontal disease in children and young adults. Periodontology 2000 2001, 26, 33-53. [CrossRef]

36. Tsai, C.-Y.; Tang, C.Y.; Tan, T.-S.; Chen, K.-H.; Liao, K.-H.; Liou, M.-L. Subgingival microbiota in individuals with severe chronic periodontitis. J. Microbiol. Immunol. Infect. 2018, 51, 226-234. [CrossRef]

37. Wang, J.; Qi, J.; Zhao, H.; He, S.; Zhang, Y.; Wei, S.; Zhao, F. Metagenomic sequencing reveals microbiota and its functional potential associated with periodontal disease. Sci. Rep. 2013, 3, 1843. [CrossRef]

38. Laksmana, T. Metagenomic Analysis of Subgingival Microbiota Following Non-Surgical Periodontal Therapy: A Pilot Study. Open Dent. J. 2012, 6, 255-261. [CrossRef]

39. Chen, H.; Liu, Y.; Zhang, M.; Wang, G.; Qi, Z.; Bridgewater, L.; Zhao, L.; Tang, Z.; Pang, X. A Filifactor alocis-centered co-occurrence group associates with periodontitis across different oral habitats. Sci. Rep. 2015, 5, 9053. [CrossRef]

40. Liu, B.; Faller, L.L.; Klitgord, N.; Mazumdar, V.; Ghodsi, M.; Sommer, D.D.; Gibbons, T.R.; Treangen, T.J.; Chang, Y.-C.; Li, S.; et al. Deep Sequencing of the Oral Microbiome Reveals Signatures of Periodontal Disease. PLoS ONE 2012, 7, e37919. [CrossRef]

41. Könönen, E.; Müller, H.-P. Microbiology of aggressive periodontitis. Periodontology 2000 2014, 65, 46-78. [CrossRef] 
42. Kumar, P.S.; Griffen, A.L.; Moeschberger, M.L.; Leys, E.J. Identification of Candidate Periodontal Pathogens and Beneficial Species by Quantitative 16S Clonal Analysis. J. Clin. Microbiol. 2005, 43, 3944-3955. [CrossRef] [PubMed]

43. Teles, R.; Teles, F.; Frias-Lopez, J.; Paster, B.; Haffajee, A. Lessons learned and unlearned in periodontal microbiology. Periodontology 2000 2013, 62, 95-162. [CrossRef]

44. Sanz, M.; Beighton, D.; Curtis, M.A.; Cury, J.A.; Dige, I.; Dommisch, H.; Ellwood, R.; Giacaman, R.; Herrera, D.; Herzberg, M.C.; et al. Role of microbial biofilms in the maintenance of oral health and in the development of dental caries and periodontal diseases. Consensus report of group 1 of the Joint EFP/ORCA workshop on the boundaries between caries and periodontal disease. J. Clin. Periodontol. 2017, 44, S5-S11. [CrossRef]

45. Kolenbrander, P.E.; Palmer, R.J.; Periasamy, S.; Jakubovics, N.S. Oral multispecies biofilm development and the key role of cell-cell distance. Nat. Rev. Microbiol. 2010, 8, 471-480. [CrossRef] [PubMed]

46. Sutherland, R.; Croydon, E.A.; Rolinson, G.N. Amoxycillin: A new semi-synthetic penicillin. Br. Med. J. 1972, 3, 13-16. [CrossRef] [PubMed]

47. Edwards, D.I. Mechanisms of selective toxicity of metronidazole and other nitroimidazole drugs. Br. J. Vener. Dis. 1980, 56, 285-290. [CrossRef] [PubMed]

48. Sgolastra, F.; Petrucci, A.; Gatto, R.; Monaco, A. Effectiveness of Systemic Amoxicillin/Metronidazole as an Adjunctive Therapy to Full-Mouth Scaling and Root Planing in the Treatment of Aggressive Periodontitis: A Systematic Review and Meta-Analysis. J. Periodontol. 2012, 83, 731-743. [CrossRef]

49. Berglundh, T.; Krok, L.; Liljenberg, B.; Westfelt, E.; Serino, G.; Lindhe, J. The use of metronidazole and amoxicillin in the treatment of advanced periodontal disease. A prospective, controlled clinical trial. J. Clin. Periodontol. 1998, 25, 354-362. [CrossRef] [PubMed]

50. Cionca, N.; Giannopoulou, C.; Ugolotti, G.; Mombelli, A. Amoxicillin and metronidazole as an adjunct to full-mouth scaling and root planing of chronic periodontitis. J. Periodontol. 2009, 80, 364-371. [CrossRef] [PubMed]

51. Dahiya, P.; Kamal, R. Hyaluronic acid: A boon in periodontal therapy. N. Am. J. Med. Sci. 2013, 5, 309. [CrossRef] [PubMed]

52. Adams, D.; Addy, M. Mouthrinses. Adv. Dent. Res. 1994, 8, 291-301. [CrossRef]

53. Goudouri, O.; Kontonasaki, E.; Lohbauer, U.; Boccaccini, A.R. Antibacterial properties of metal and metalloid ions in chronic periodontitis and peri-implantitis therapy. Acta Biomater. 2014, 10, 3795-3810. [CrossRef] [PubMed]

54. Chen, C.K.C.; Wilson, M.E. Eikenella corrodens in Human Oral and Non-Oral Infections: A Review. J. Periodontol. 1992, 63, 941-953. [CrossRef]

55. Baghban, A.; Gupta, S. Parvimonas micra: A rare cause of native joint septic arthritis. Anaerobe 2016, 39, 26-27. [CrossRef]

56. Rams, T.E.; Feik, D.; Listgarten, M.A.; Slots, J. Peptostreptococcus micros in human periodontitis. Oral Microbiol. Immunol. 1992, 7, 1-6. [CrossRef] [PubMed]

57. Santos, F.A.; Bastos, E.M.A.; Rodrigues, P.H.; de Uzeda, M.; de Carvalho, M.A.R.; de Macedo Farias, L.; Andrade Moreira, E.S. Susceptibility of Prevotella intermedia/Prevotella nigrescens (and Porphyromonas gingivalis) to Propolis (Bee Glue) and other Antimicrobial Agents. Anaerobe 2002, 8, 9-15. [CrossRef]

58. Han, Y.W. Fusobacterium nucleatum: A commensal-turned pathogen. Curr. Opin. Microbiol. 2015, 23, 141-147. [CrossRef] [PubMed]

59. Jacinto, R.C.; Montagner, F.; Signoretti, F.G.C.; Almeida, G.C.; Gomes, B.P.F.A. Frequency, Microbial Interactions, and Antimicrobial Susceptibility of Fusobacterium nucleatum and Fusobacterium necrophorum Isolated from Primary Endodontic Infections. J. Endod. 2008, 34, 1451-1456. [CrossRef]

60. Raja, M. Aggregatibacter Actinomycetemcomitans-A Tooth Killer? J. Clin. Diagn. Res. 2014, 8, 13-16. [CrossRef] [PubMed]

61. Oettinger-Barak, O.; Dashper, S.G.; Catmull, D.V.; Adams, G.G.; Sela, M.N.; Machtei, E.E.; Reynolds, E.C. Antibiotic susceptibility of Aggregatibacter actinomycetemcomitans JP2 in a biofilm. J. Oral Microbiol. 2013, 5, 20320. [CrossRef] 
62. How, K.Y.; Song, K.P.; Chan, K.G. Porphyromonas gingivalis: An Overview of Periodontopathic Pathogen below the Gum Line. Front. Microbiol. 2016, 7, 1-14. [CrossRef] [PubMed]

63. Jacinto, R.C.; Gomes, B.P.F.A.; Shah, H.N.; Ferraz, C.C.; Zaia, A.A.; Souza-Filho, F.J. Incidence and antimicrobial susceptibility of Porphyromonas gingivalis isolated from mixed endodontic infections. Int. Endod. J. 2006, 39, 62-70. [CrossRef] [PubMed] 


\section{III.}




\section{Electrospun PLA Fibers Containing Metronidazole for Periodontal Disease}

This article was published in the following Dove Press journal:

Drug Design, Development and Therapy

\author{
Mária Budai-Szűcs' \\ Attila Léber' \\ Lu Cui $\mathbb{1}^{2,3}$ \\ Muriel Józó (iD ${ }^{2,3}$ \\ Péter Vályi ${ }^{4}$ \\ Katalin Burián ${ }^{5}$ \\ Balázs Kirschweng (iD ${ }^{2,3}$ \\ Erzsébet Csányi (D) \\ Béla Pukánszky (iD) 2,3 \\ 'Institute of Pharmaceutical Technology \\ and Regulatory Affairs, Faculty of \\ Pharmacy, University of Szeged, Szeged, \\ Hungary; ${ }^{2}$ Laboratory of Plastics and \\ Rubber Technology, Department of \\ Physical Chemistry and Materials Science, \\ Budapest University of Technology and \\ Economics, Budapest H-I52I, Hungary; \\ ${ }^{3}$ Institute of Materials and Environmental \\ Chemistry, Research Centre for Natural \\ Sciences, Hungarian Academy of \\ Sciences, Budapest H-1519, Hungary; \\ ${ }^{4}$ Department of Periodontology, Faculty \\ of Dentistry, University of Szeged, \\ Szeged, Hungary; ${ }^{5}$ Institute of Clinical \\ Microbiology, Faculty of Medicine, \\ University of Szeged, Szeged, Hungary
}

Correspondence: Mária Budai-Szűcs Institute of Pharmaceutical Technology and Regulatory Affairs, Faculty of Pharmacy, University of Szeged, Eötvös utca 6 , Szeged 6720 , Hungary

Tel $+3662545-573$

Fax $+3662545-571$

Email maria.szucs@pharm.u-szeged.hu
Purpose: Electrospun PLA fiber devices were investigated in the form of fiber mats and disks. Metronidazole was used as an active agent; its concentration was 12.2 and $25.7 \mathrm{wt} \%$ in the devices. Methods: The structure was studied by X-ray diffraction and scanning electron microscopy, drug release by dissolution measurements, while the antimicrobial efficiency was tested on five bacterial strains.

Results: The XRD study showed that the polymer was partially crystalline in both devices, but a part of metronidazole precipitated and was in the form of crystals among and within the fibers. Liquid penetration and dissolution were different in the two devices, they were faster in disks and slower in fiber mats, due to the morphology of the device and the action of capillary forces. Disks released the drug much faster than fiber mats. Although the release study indicated fast drug dissolution, the concentration achieved a plateau value in 24 hrs for the disks; the inhibition effect lasted much longer, 13 days for bacteria sensitive to metronidazole. The longer inhibition period could be explained by the slower diffusion of metronidazole located inside the fibers of the device.

Conclusion: The results suggest that the devices may be effective in the treatment of periodontitis.

Keywords: drug release devices, fiber mats, disks, morphology, dissolution, capillary forces, diffusion, antimicrobial, inhibition

\section{Introduction}

Periodontitis is an inflammatory disease resulting from the overgrowth of subgingival polymicrobial community in susceptible hosts affecting the tissues surrounding the teeth. The inflammation of periodontal tissue causes bone destruction by osteoclastic resorption together with tissue destruction, and the detachment of junctional epithelium result in periodontal pocket formation. Conditions in the periodontal pocket are suitable for bacterial proliferation and result in dysbiosis owing to a breakdown in host-microbe homeostasis. ${ }^{1,2}$ This disease is the most prevalent reason for tooth loss among adults. ${ }^{3}$

The elimination of biofilm and/or hard deposits (mineralized biofilm) is the cornerstone of periodontal treatment. The management of periodontal disease includes the mechanical removal of biofilm with or without the adjunctive use of systemic antibiotics. ${ }^{4}$ Systemically administered antibiotics may not be present in sufficient concentration in the periodontal pocket; therefore, they are often ineffective, while the common side effects of antimicrobial therapy could occur. ${ }^{5}$

Numerous publications published in the open literature report investigations on local delivery systems containing antimicrobial drugs, which - alone or in combination 
with other dental procedures - may result in a more efficient treatment. $^{5-10}$ The local administration of delivery systems with incorporated antibiotics is a promising approach to treating periodontitis. The introduction of devices containing antibiotics into the periodontal pockets could result in efficient therapy with limited side effects and reduced risk of developing drug-resistant microbes. The comparison of local to systemic drug delivery shows that 100 -fold larger concentrations of the antimicrobial agents can be achieved at subgingival sites, which can lead to a more efficient therapy. ${ }^{11}$ Numerous local drug delivery systems such as fibers, strips, films, injectable gels, micro- and nanoparticulate systems, vesicular systems, and in-situ forming implants were developed for this purpose during the last decades. ${ }^{5-10}$

The electrospinning technique has received considerable attention lately because it is an affordable, cost-effective and easy-to-use technology for creating nanofibrous scaffolds for tissue engineering purposes, as well as drug delivery systems and wound dressings. ${ }^{12-15}$ The advantages of electrospun drug delivery systems are numerous: i) large drug loading capacity (up to $60 \%$ ), ${ }^{16}$ ii) different polymers may be applied depending on the compatibility of the active pharmaceutical ingredients, iii) the process is simple and cost-effective, ${ }^{17}$ and iv) the modulation of drug release can be achieved as well. ${ }^{18,19}$

Sustained drug release can be achieved by both nonbiodegradable and biodegradable polymers. Biodegradable polyesters such as PLA, polyglycolic acid (PGA), poly (lactic-co-glycolic) acid (PLGA), and polycaprolactone (PCL) have already been applied ${ }^{18}$ for this purpose. Information about research describing polymer or biopolymer-based electrospun delivery systems containing different antimicrobial agents, which may be applied with success for the treatment of periodontitis, is available in the literature. ${ }^{20-25}$ Fiber mats fabricated using the electrospinning method may be suitable for the treatment of periodontal disease and they could provide prolonged drug release. ${ }^{20}$

The goal of the current work was to develop and investigate locally administrable, PLA-based, metronidazolecontaining devices for the treatment of periodontal disease. The devices were composed of electrospun fiber mats as received from the spinning process and compressed disks prepared from the mats. The drug used was metronidazole, which is known to be an active pharmaceutical ingredient (API) with an undesirable bitter taste, hindering its local application as a drug for periodontal treatment. It is expected that the encapsulation of metronidazole into a hydrophobic polymer matrix, sustained release, and probably a lower effective concentration in the oral cavity might eliminate this problem. We assumed that mats enable the free flow of the exudate in the periodontal pocket. On the other hand, disks provide drug delivery devices with a standardized geometry (surface, width) and equal drug content and facilitate the treatment of tight pockets. Accordingly, we analyzed the effect of drug concentration and the form of the delivery device on the kinetics of drug release in detail. Practical consequences are briefly mentioned in the final section of the paper.

\section{Materials and Methods \\ Materials}

Spinning solution was made by dissolving polylactic acid (PLA) (Ingeo 4032D type, NatureWorks LLC., Minnetonka, $\mathrm{MN}$, USA) in a mixture of dichloromethane and dimethyl sulfoxide (Molar Chemicals Ltd., Halásztelek, Hungary). For the spinning of fibers containing an active agent, metronidazole (Ph. Eur. 8., Hungaropharma Plc., Budapest, Hungary) was also dissolved in the same solvent mixture in different concentrations.

For in vitro drug diffusion measurements, a $p \mathrm{H}=7.4$ phosphate-buffered saline (PBS) solution was used. The buffer solution was prepared by dissolving $8 \mathrm{~g} / \mathrm{dm}^{3} \mathrm{NaCl}$, $0.2 \mathrm{~g} / \mathrm{dm}^{3} \mathrm{KCl}, 1.44 \mathrm{~g} / \mathrm{dm}^{3} \mathrm{Na}_{2} \mathrm{HPO}_{4} \cdot 2 \mathrm{H}_{2} \mathrm{O}$, and $0.12 \mathrm{~g} /$ $\mathrm{dm}^{3} \mathrm{KH}_{2} \mathrm{PO}_{4}$ (Ph. Eur. 8., Hungaropharma Plc., Budapest, Hungary) in distilled water. The $\mathrm{pH}$ was adjusted to 7.4 by adding an adequate amount of $0.1 \mathrm{M} \mathrm{HCl}$ to the solution.

\section{Methods}

\section{Fiber Spinning}

PLA fibers containing various amounts of metronidazole were prepared by coelectrospinning, which is a widely used and cost-effective method to produce nanofibers especially for tissue engineering and regenerative medicine. ${ }^{26}$ The fibers were spun at ambient temperature at $15-\mathrm{kV}$ voltage, $10-\mathrm{cm}$ collector distance, and a feeding rate of $3 \mathrm{~cm}^{3} / \mathrm{hr}$. The solvent used was the $80 / 20 \mathrm{vol} \%$ mixture of dichloromethane and dimethyl sulfoxide. The solution contained the polymer in $10 \mathrm{wt} \%$, while the amount of the active component was changed from 0 to $3 \mathrm{wt} \%$ of the solution. The random array of fibers was compressed to disks of 13-mm diameter for further testing.

\section{Sample Preparation}

The metronidazole concentration of the fibers was 12.2 and $25.7 \mathrm{wt} \%$, the latter corresponding to the metronidazole 
concentration of the saturated spinning solution. Neat electrospun fiber mats taken directly from the aluminum collector film and round-shaped, compressed disks were used for the analysis of release kinetics and microbial activity. The disks were obtained by compressing approximately $10-15 \mathrm{mg}$ of the neat fiber under $1 \mathrm{kN}$ pressure for $30 \mathrm{~s}$ in a pellet die of 13-mm diameter (Specac Atlas Manual Hydraulic Press 15T and Specac 13 mm Pellet Press Die, Specac Ltd., Orpington, Kent, UK).

\section{Scanning Electron Microscopy}

The appearance of the disks and fibers and the diameter of the latter were investigated by scanning electron microscopy using a Jeol JSM 6380 LA apparatus. The micrographs were recorded at different magnifications using $15-\mathrm{kV}$ acceleration voltage. Before viewing, the fibers were sputtered with gold. The determination of fiber thickness was done with the Image Pro Plus 6 software.

\section{X-Ray Diffraction Analysis}

A Bruker D8 Advance diffractometer (Bruker AXS GmbH, Karlsruhe, Germany) with $\mathrm{CuK} \alpha$ radiation $(\lambda=1.5406 \AA)$ was used for the XRD analysis. The samples were scanned at $40 \mathrm{kV}$ and $20 \mathrm{~mA}$ from $3^{\circ}$ to $40^{\circ} 2 \theta$ angle at a scanning rate of $0.1 \%$ and a step size of $0.01^{\circ}$.

\section{Penetration of the Aqueous Medium, Wetting}

The behavior of the devices in contact with water was studied with an OCA Contact Angle System (Dataphysics OCA 20, Dataphysics Inc., GmbH, Germany). The contact angle of water droplets was determined on the neat electrospun fibers, the disks prepared from them as described above, and on a metronidazole pastille of the same size. The measurement was done with a water droplet of $10 \mu \mathrm{L}$ volume, which was dropped from a calibrated syringe onto the surface of the various devices. Contact angle was measured instantly after the placement of the droplet, as well as 10 and 20 mins afterward. Five parallel measurements were done on each sample.

\section{In vitro Drug Release Study}

The in vitro drug release profiles of the nanofibers were determined by the measurement of dissolution followed by UV-Vis spectroscopy. Disks (0.014-0.017 g) and neat fiber mats $(0.012-0.016 \mathrm{~g})$ were weighed and put into $7.5 \mathrm{~mL}$ of $\mathrm{pH}=7.4$ PBS solution thermostated at $37^{\circ} \mathrm{C}$. Samples of $1.0 \mathrm{~mL}$ volume were taken at $0.5,1,4,6,10$, 24, 30, 48, 72, 96, and $196 \mathrm{hrs}$ and replaced with $1.0 \mathrm{~mL}$ of fresh PBS solution. Drug release was followed for 7 days. The concentration of the released metronidazole was determined by UV-Vis spectrophotometry (Helios $\alpha$ Thermospectronic UV-spectrophotometer v4.55, Unicam: Thermo Fisher Scientific, Waltham, MA) at $318 \mathrm{~nm}$. Blank samples (PLA fiber mats and disks) were also treated in the same way as reference, but in their case no absorbance was detected in the wavelength range of 200-900 nm with spectrophotometric analysis.

\section{Antimicrobial Test}

The antibacterial efficiency of the disks containing 12.2 or $25.7 \mathrm{wt} \%$ metronidazole was determined as well. The control strains of five bacteria were used in the study: Fusobacterium nucleatum (ATCC ${ }^{\circledR} 25586^{\mathrm{TM}}$ ), Parvimonas micra (ATCC 33270 ${ }^{\mathrm{TM}}$ ), Eikenella corrodens (ATCC 23834TM), Aggregatibacter actinomycetemcomitans (ATCC 29524TM), and Prevotella intermedia (118710). All measurements were carried out according to the same protocol. A bacterial suspension of one McFarland standard concentration, which is equivalent to approximately $3 \times 10^{8}$ colonyforming units $/ \mathrm{mL}$ in the suspension, was freshly prepared with normal saline solution. Equivalent portions of the suspensions were then spread onto a horse blood agar plate and disks of equal weight were placed on it. The diameter of the inhibition zone was measured after $24 \mathrm{hrs}$ of incubation in an anaerobic chamber. The disks were then put again on a new horse blood agar plate, also inoculated with a bacterial suspension of one McFarland standard concentration freshly made from the bacterial strains mentioned above. The plates were then put into an anaerobic chamber for $24 \mathrm{hrs}$. The procedure was repeated until no inhibition zone could be detected, or for a maximum of 14 days. Three parallel measurements were carried out for every formulation and bacterial strain.

\section{Statistical Analysis}

The results of the in vitro drug release study were analyzed statistically with GraphPad Prism version 5 software. Twoway ANOVA analysis was used with Bonferroni post-tests. A level of $p \leq 0.05$ was considered as significant, $p \leq 0.01$ as very significant, and $\mathrm{p} \leq 0.001$ as highly significant.

\section{Results and Discussion}

The results are discussed in several sections. First, results related to the structure of the devices are presented and then the penetration of the dissolution medium into them is analyzed. Drug release and the results of the microbiological testing are discussed in the last two sections together with a few remarks on practical relevance. 


\section{Structure}

PLA has a regular molecular structure and can crystallize under appropriate conditions, and metronidazole is also a crystalline material. The solubility of the drug in the polymer and its diffusion into the surrounding medium depend on the structure of the components and on their distribution. Metronidazole is a water-soluble, polar substance, the solubility of which is small in the polymer. Accordingly, some of the drugs can be dissolved, but another part precipitates and is located among or inside the fibers as crystals. Structure determines drug release and its control allows the regulation of efficiency through modified drug release.

The structure was studied by XRD analysis and SEM microscopy. The crystallization of PLA is slow; thus, products prepared from this polymer are usually amorphous. On the other hand, the evaporation rate and the possible presence of residual solvent might result in the crystallization of the polymer. The XRD study showed that the fibers were partially crystalline at the time of the measurements and the release, as well as during the microbiological measurements. As expected, the compression of the mat into disc did not change the structure.

The incorporation of metronidazole may change the structure of the polymer. The drug may act as a nucleating agent, but it can change the rate of crystallization as well. The XRD traces of metronidazole and those of the fiber mat containing 12.2 and $25.7 \mathrm{wt} \%$ of the drug are presented in Figure 1. The characteristic reflections of metronidazole can be detected in the traces and their intensity increases with increasing concentration. Obviously, not the entire amount of

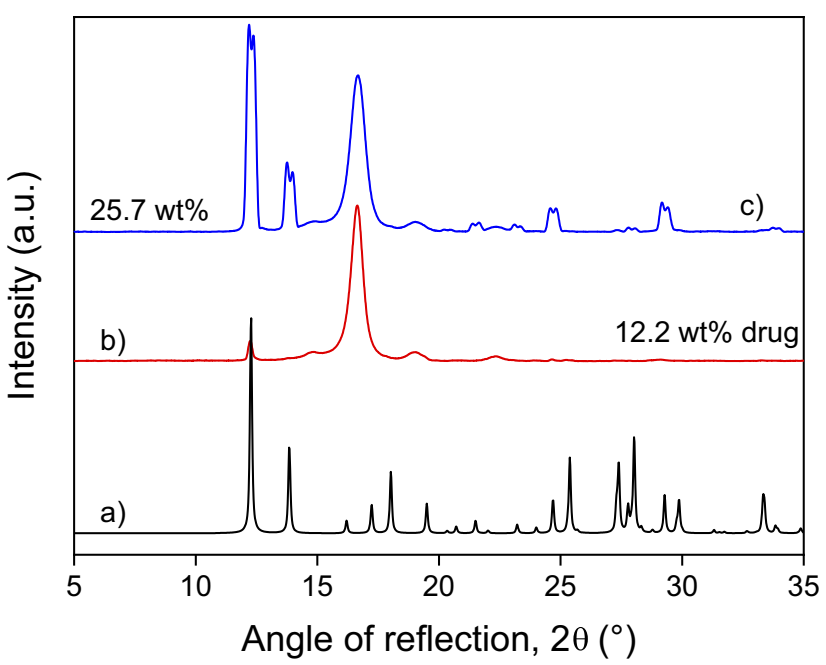

Figure I Influence of metronidazole content on the structure of PLA fiber mats, where: a) neat metronidazole, b) $12.2 \mathrm{wt} \%$, and c) $25.7 \mathrm{wt} \%$ drug. the drug added to the spinning solution is dissolved in the polymer, but it precipitates during fiber spinning and is dispersed in crystalline form among and/or inside the fibers. The location and physical form of metronidazole may influence drug release significantly, thus determining both the efficiency of the device and its lifetime, the length of the active period.

The XRD traces of disks containing $25.7 \mathrm{wt} \%$ metronidazole are presented in Figure 2 before and after drug release (dissolution). The intensity of characteristic peaks belonging to metronidazole decreases during the dissolution experiment as an effect of drug release. It can also be seen that some metronidazole remains in the device even after the dissolution experiment, which confirms our earlier assumption that some of the drug precipitates during fiber spinning and crystals are located not only among, but also within the fibers. The release of this latter part of the drug is quite slow and does not take place in the timescale of the dissolution experiment.

The structure of the fiber mat and the disks was also studied by scanning electron microscopy. Micrographs showing the differences in structure are presented in Figure 3. The mat consists of loose fibers with considerable space among individual fibers (Figure 3A). One would expect fast penetration and easy flow of the fluid used for dissolution and thus very fast release of the drug. The scrutiny of micrographs recorded on mats reveals the presence of metronidazole crystals among the fibers. The structure of a disk is shown in the micrograph of Figure 3B. The disk has a much more compact structure, voids are smaller, and the fibers are close to each other. The presence of

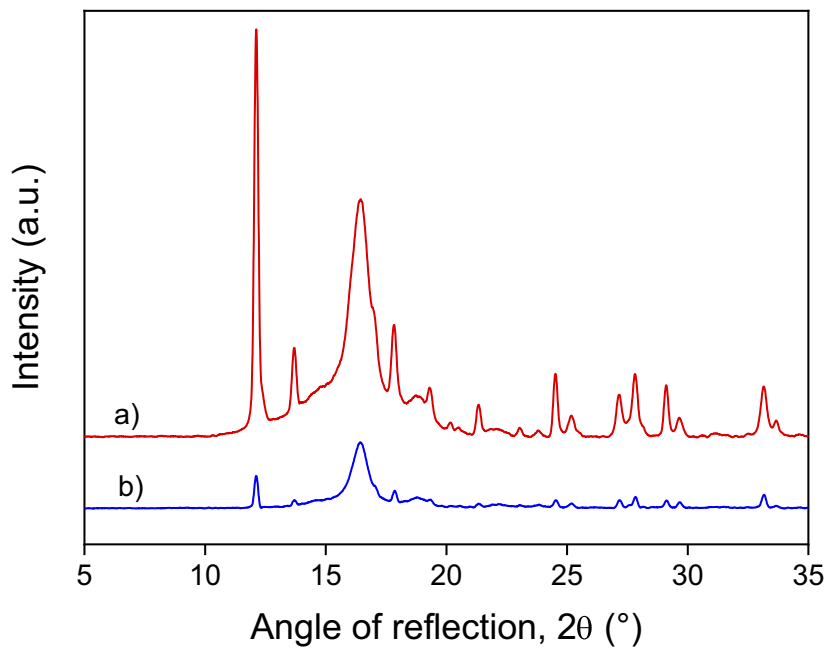

Figure 2 Effect of the dissolution of the drug on the structure of disks prepared from electrospun fibers by compression. Metronidazole content: $25.7 \mathrm{wt} \%$. a) before and b) after the dissolution experiment. 

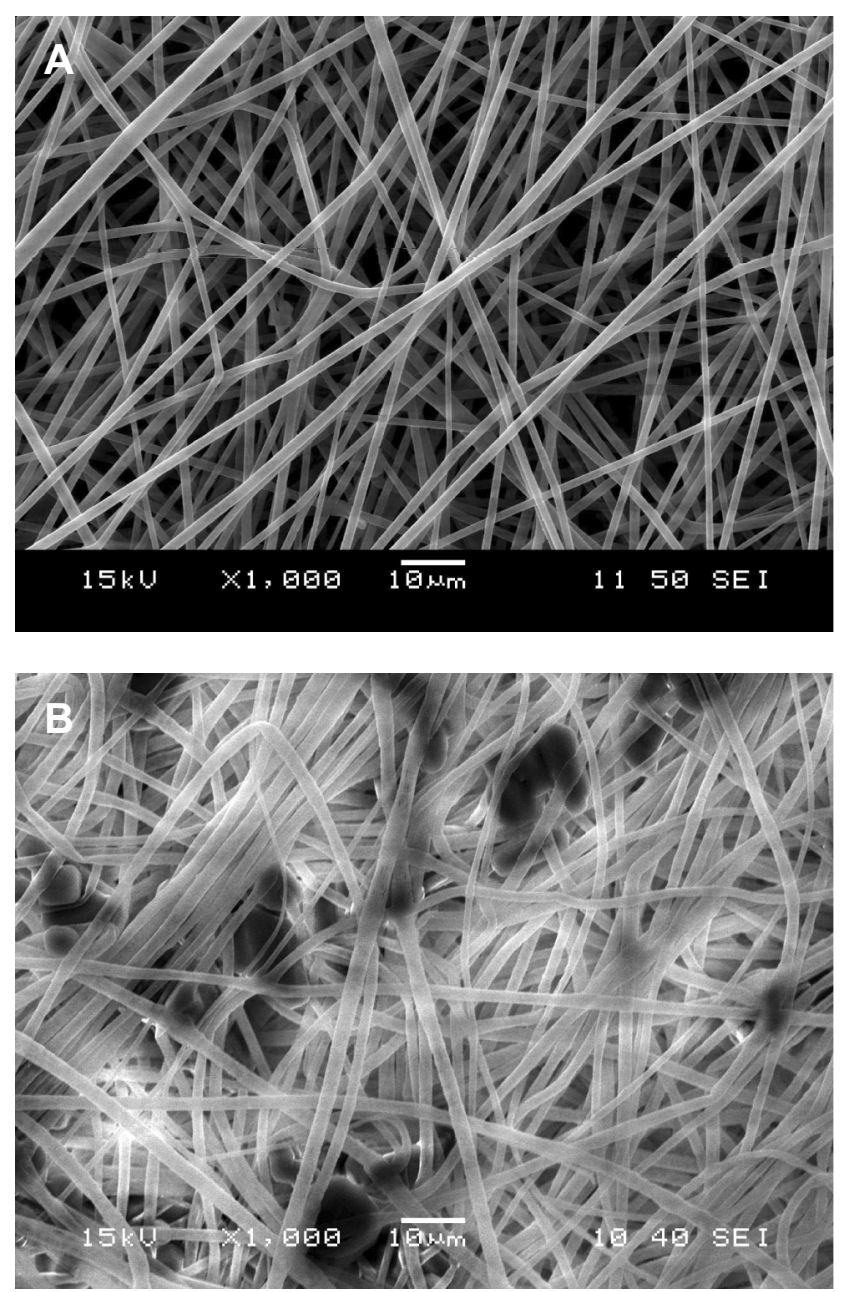

Figure 3 SEM micrographs recorded on the PLA devices studied. A) fiber mat, B) compressed disk. The black dots are crystalline metronidazole particles in Figure 3B.

metronidazole crystals among the fibers is more obvious in this case. The SEM study verified the conclusions drawn from the XRD measurements and proves that a part of the drug is distributed in crystal form among and probably also within the fibers.

\section{Penetration of the Aqueous Medium, Wetting}

In order to obtain some idea about the penetration of the dissolution medium into the devices prepared, the contact angle of water was measured on them as a function of time. The results are presented in Table 1. Interpretation is difficult because of the complexity of the system and the related processes. Contact angle is usually measured by placing a droplet of a liquid onto a smooth, stable surface. In our case, the surface used for the measurement is neither stable nor smooth. Metronidazole dissolves in water, while both
Table I Contact Angles Measured (Mean and SD Values) on a Metronidazole Pastille and on Drug Release Devices (Fiber Mats, Disks) at Various Times

\begin{tabular}{|l|l|l|l|l|}
\hline Device & \multirow{2}{*}{$\begin{array}{l}\text { Drug } \\
\text { Content } \\
\end{array}$} & \multicolumn{3}{|l|}{ Contact Angle (') After Time (Min) } \\
\cline { 3 - 5 } & (wt\%) & 0 & 10 & 20 \\
\hline MZ pastille & 100 & $32.5 \pm 1.8$ & - & - \\
\hline Fiber mat & 0 & $125.3 \pm 3.8$ & $123.2 \pm 4.0$ & $121.4 \pm 0.8$ \\
& 12.2 & $110.8 \pm 3.1$ & $104.6 \pm 5.3$ & $96.0 \pm 8.9$ \\
& 25.7 & $117.3 \pm 7.2$ & $112.3 \pm 9.5$ & $105.8 \pm 12.2$ \\
\hline Disk & 0 & $62.5 \pm 1.6$ & $48.7 \pm 0.5$ & - \\
& 12.2 & $66.9 \pm 1.4$ & $46.4 \pm 0.3$ & - \\
& 25.7 & $64.3 \pm 0.5$ & $47.0 \pm 0.2$ & - \\
\hline
\end{tabular}

the fiber mats and the compressed disks have rough, porous surfaces (see Figure 3) into which the liquid may penetrate. Accordingly, the contact angle measured depends on the rate of dissolution in the case of metronidazole, and on surface tension and surface morphology in the case of the two devices. Pore size and capillary forces also play an important role in the determination of the value of the contact angle, but also in drug release. Since the surface tension of the components is constant and we do not expect large changes in the surface tension of PLA as an effect of the dissolution of some metronidazole, the major factors must be morphology, porosity, and capillary forces.

According to the results of Table 1, the contact angle of water on the metronidazole pastille is small, which is not very surprising since metronidazole dissolves in water. Dissolution is confirmed by the fact that the contact angle could not be measured after 10 mins of forming the droplet. Contrary to metronidazole, the contact angle of the droplet placed onto the fiber mat is quite large, indicating poor wetting and penetration. Contact angle does not change with metronidazole content; the differences are caused by changes in morphology and the standard deviation of the measurements. Contact angles showed just a slight systematic decrease with time for the neat fiber mats, indicating that water can slowly penetrate into the mat.

The contact angle measured on the compressed disks is much smaller and it is independent of the concentration of metronidazole as well. It decreases considerably with time and could not be measured after 10 mins at all, showing that the water droplet placed onto the disk disappeared in this time interval. The difference in the contact angles measured on the fiber mat and the compressed disk proves 
that the primary factor determining aqueous media penetration is morphology and this factor is expected to determine the extent and rate of drug release as well.

The apparent contradiction that water cannot penetrate so fast into the loose fiber mats, while it does into the discs, should be considered. Capillary forces depend on the interaction of the liquid and the capillary and also on the size of the capillary, i.e., on pore size, in our case. Water climbs in a glass capillary, but the level of mercury decreases in it. The surface tension of water is $72 \mathrm{~mJ} / \mathrm{m}^{2}$, while that of mercury is $486 \mathrm{~mJ} / \mathrm{m}^{2}$; thus, the first wets glass, while the other does not. In our case, the surface tension of water is still the same $\left(72 \mathrm{~mJ} / \mathrm{m}^{2}\right)$ as mentioned above and that of PLA is around $40 \mathrm{~mJ} / \mathrm{m}^{2}{ }^{27-29}$ Accordingly, water does not wet PLA fibers and cannot penetrate into the devices easily. Compression obviously changed the pore structure of the device (see Figure 3), which decreases capillary forces and helps penetration. As an effect of changing morphology and penetration, we may expect a faster release of the drug from the disk than from the fiber mat.

\section{In vitro Drug Release}

The release of the drug incorporated into the devices prepared from electrospun fibers is a complex process and depends on several factors. As the results of the XRD measurements (Figures 1 and 2) and the SEM micrographs (Figure 3) showed the drug, metronidazole in this case, is incorporated into the devices in various forms: as precipitated crystals within and among the fibers and as dissolved molecules in PLA. The dissolution of these forms must be different. The PBS solution must penetrate the device, dissolve the crystals and diffuse out into the surrounding medium. On the other hand, dissolved metronidazole must diffuse out of the PLA fibers into the surrounding medium. The solubility of metronidazole is small in PLA, and diffusion is driven by concentration difference, which is also small or even negative due to the large drug concentration of the surrounding solution because of the dissolution of the crystals. Consequently, the main factor determining the dissolution of metronidazole from the devices, i.e., drug release, is the penetration and flow of the PBS solution. This is different for the two devices, mats and disks; thus dissimilar drug release is expected from them.

The time dependence of dissolution is presented in Figure 4 for the fiber mats and the disks at two different concentrations. In view of the observations presented

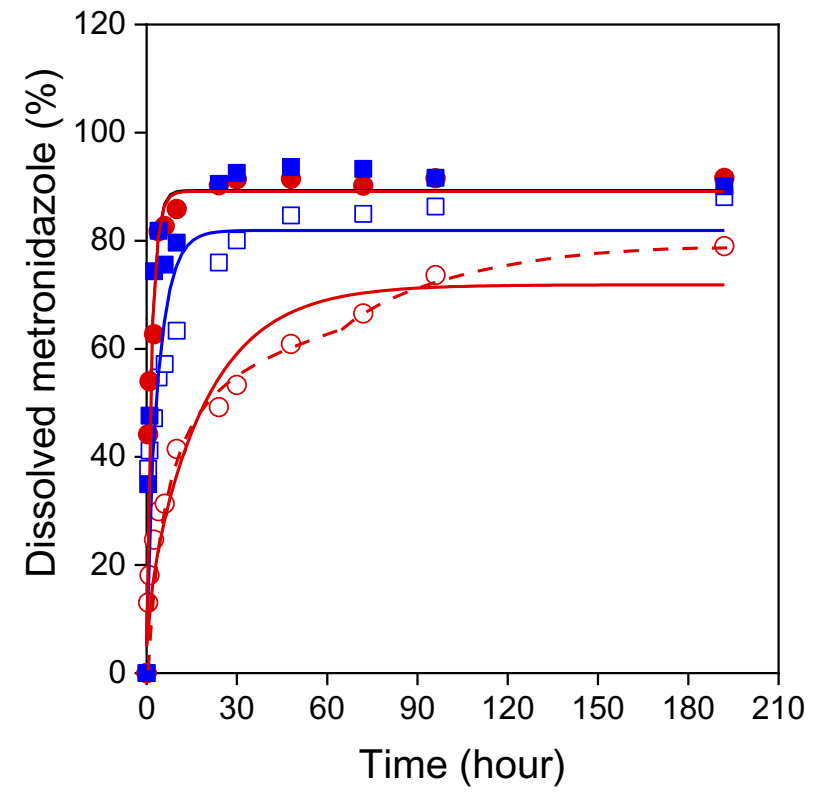

Figure 4 Dissolution of metronidazole from fiber mats and disks. Effect of drug content. Symbols: $(\square, \bullet) 12.2 \mathrm{wt} \%,(\circ, \bullet) 25.7 \mathrm{wt} \%$ metronidazole; empty symbols: fiber mat, full symbol: disk; solid lines are fitted functions, while the broken line shows the two-step process.

in Section 3.2, some of the results were expected. Compressed disks release the drug much faster than fiber mats because of the larger and faster penetration of the aqueous medium into the pores of the device. However, the fact that dissolution is independent of the initial concentration of metronidazole in the device is somewhat surprising. The fast penetration and dissolution of metronidazole in the PBS solution can result in the independence of concentration. The diffusion of the liquid containing the dissolved drug into the surrounding medium may be the rate-determining step of dissolution in this case.

In the case of the fiber mats, the rate of dissolution depends on concentration, however, not as expected, i.e., faster rate at larger concentration, but in the opposite way. The slower release of the drug from the mats can be understood easily if we consider the difference in the penetration of the aqueous medium (see Table 1). The effect of concentration, on the other hand, is difficult to explain. Obviously, the dissolution of the drug in the PBS solution and its diffusion into the surrounding medium are the rate-determining steps in this case. However, the presence of the drug cannot influence the diffusion rate much; thus, dissolution must be dissimilar at the two concentrations of metronidazole. The larger drug concentration probably results in larger precipitated crystals, which leads to slower dissolution and release. 
The rate of dissolution can be estimated qualitatively from the correlations presented in Figure 4. According to this evaluation, plateau concentrations are reached after 24 hrs for the disks, and after 48 or 96 hrs for the fiber mats. However, time dependence can be evaluated quantitatively if appropriate functions are fitted to the experimental data. The dissolution and the diffusion of the drug are determined by Fick's laws. Fick's equations can be solved numerically or they can be expressed analytically using simplifications. ${ }^{30}$ Two main approaches are used in practice, those describing the first part of the function plotting experimental results as the function of the square root of time, or those which use an exponential function. This approach gives a more accurate estimate at long times and it allows the estimation of the overall rate of dissolution and the maximum amount of dissolved material at infinite time (if the shape and structure of the device do not change, e.g.: fiber degradation). We followed the latter approach and fitted the function of Equation 1 to the experimental results:

$M_{t}=M_{\infty}\left\{1-\frac{8}{\pi^{2}}\left[\exp (-a t)+\frac{1}{9} \exp (-9 a t)+\frac{1}{25} \exp (-25 a t)\right]\right\}$

where $M_{t}$ and $M_{\infty}$ are the dissolved amount of drug at time $t$ and at infinite time, respectively, and $a$ is the overall rate of dissolution. The fitted functions are presented in Figure 4 as solid lines. The parameters calculated from the fitting are collected in Table 2.

Results presented in Table 2 confirm our qualitative evaluation and show that dissolution is much faster from the disk than from the fiber mat (see parameter $a$ ). It also confirms the composition dependence observed. The comparison of the predicted amount of drug dissolved at infinite time $\left(M_{\infty}\right)$ indicates that a considerable amount of drug, $10-30 \%$ remains in the devices after the dissolution experiment even in the case of the disks. Moreover, a closer comparison of the fitted lines and the measured values indicates that dissolution cannot be described with a single process; it consists of at least two steps, a faster

Table 2 Parameters Characterizing the Kinetics of Dissolution Determined by Fitting Equation I to the Experimental Results

\begin{tabular}{|l|l|l|l|l|}
\hline Drug Content (wt\%) & Form & $\mathbf{a}(\mathbf{I} / \mathbf{h r})$ & $\mathbf{M}_{\infty}(\%)$ & $\mathbf{R}^{\mathbf{2 a}}$ \\
\hline 12.2 & Mat & 0.233 & 81.9 & 0.8855 \\
25.7 & Mat & 0.051 & 71.8 & 0.9634 \\
12.2 & Disk & 0.581 & 89.4 & 0.9449 \\
25.7 & Disk & 0.556 & 89.1 & 0.9650 \\
\hline
\end{tabular}

Notes: ${ }^{a}$ Determination coefficient showing the goodness of the fit. one at the beginning of the experiment and another one proceeding at a slower rate. This two-step process is demonstrated especially well by the results obtained on the fiber mat containing $25.7 \mathrm{wt} \%$ metronidazole (see $\Delta$ series in Figure 4). The two steps demonstrated by the broken line in the figure might be explained by the dissolution of the different forms of the drug; crystals located among the fibers dissolve much faster than dissolved metronidazole or crystals precipitated within the fibers. In this case, significant differences could be observed (from 2.5 to 6 hrs: $\mathrm{p} \leq 0.001$; from 6 to $30 \mathrm{hrs:} \mathrm{p} \leq 0.01$; and from 30 to 72 hrs: $p \leq 0.05$ ) between the drug release of fiber mats and disks. The different rates allow the regulation of the amount of the drug as a function of time and also the active lifetime of the device. Controlling the form of the drug in the device and the rate of water diffusion into the electrospun porous fiber network might be an efficient strategy to control drug release. $^{31,32}$

\section{Antimicrobial Activity}

The oral cavity and/or various dental surfaces may be inhabited by numerous strains of pathogen bacteria. Personal features, dietary habits, or even habitation could have an influence on the composition of the oral microbiota. ${ }^{33,34}$ The contribution of different bacterial strains to the initiation and progression of periodontitis is under serious investigation. ${ }^{33-41}$ However, the task seems to be almost impossible because of the large number of various microorganism species, difficulties of cultivation, and the fact that only a small part of the bacteria present in the subgingival cavity could be linked to the pathogenesis of the disease. ${ }^{42}$ Pathogens playing a role in the formation and progression of periodontal disease can be subdivided into three groups. The first category strains are able to stick to dental surfaces, the bacteria in the second group form a bridge between the first and the third category, while the bacteria of the third group are responsible for bacterial biofilm formation (Socransky and Haffajee, 2002). The following strains may initiate disease formation: Porphyromonas gingivalis, P. intermedia, Bacteroides forsythus, A. actinomycetemcomitans, Treponema denticola, Tannerella forsythia, F. nucleatum, Fusobacterium periodonticum, Prevotella nigrescens, P. micra, Campylobacter gracilis, Campylobacter rectus, Campylobacter showae, Eubacterium nodatum, Streptococcus constellatus, E. corrodens, Streptococcusspp., etc. ${ }^{33,42-44}$

Only disks were included in the microbiological study, as uniform shape, weight, and thickness could not have been 
achieved with the fiber mats. The delivery systems evaluated contained 12.2 and $25.7 \mathrm{wt} \%$ metronidazole. Five different bacterial strains were used in this investigation: E. corrodens, P. intermedia, P. micra, F. nucleatum, and A. actinomycetemcomitans. E. corrodens is a facultative anaerobic, gramnegative bacterium and a human (mostly oral) pathogen. E. corrodens is reported to be unsusceptible to metronidazole. $^{45} P$. micra is a gram-positive, anaerobic coccus and part of the normal human gastrointestinal flora. ${ }^{46}$ Literature data suggest that this strain is not susceptible to metronidazole either. ${ }^{47} P$. intermedia is a black pigmented, gramnegative anaerobic bacterium. It is often connected with oral and subgingival diseases. Metronidazole is efficient against $P$. intermedia. ${ }^{48} F$. nucleatum, which is a mostly oral and periodontal anaerobic pathogen bacterium, can be related to several human diseases. This strain seems to be sensitive to metronidazole. ${ }^{49,50}$

A facultatively anaerobic gram-negative bacterium, A. actinomycetemcomitans, can often be linked to periodontal disease and oral infections. Susceptibility to metronidazole is reported to be weak. ${ }^{51,52}$

The results of the microbiological study are presented in Figure 5. The duration of the antimicrobial effect is apparently independent of concentration; it is the same for disks with 12.2 and $25.7 \mathrm{wt} \%$ metronidazole content

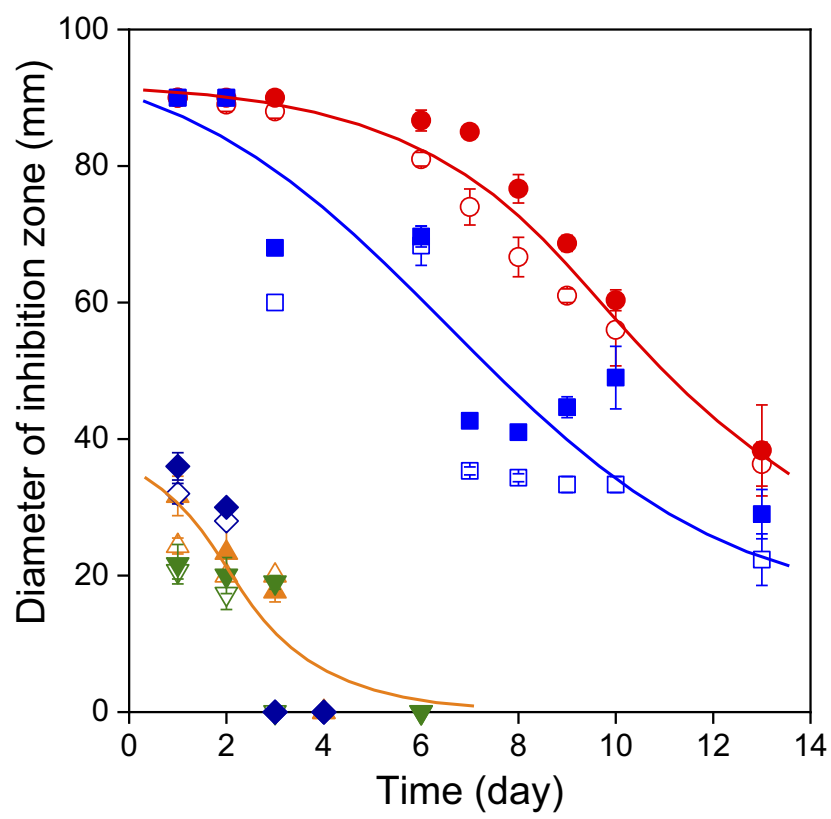

Figure 5 Inhibition zones (mean and SD values) developing in the presence of different anaerobic pathogen bacteria of the oral cavity using disks, which contain various amounts of metronidazole. Symbols: $(\circ)$ P. intermedia, ( $\square)$ F. nucleatum, $(\Delta)$ A. actinomycetemcomitans, $(\nabla)$ P. micra, $(\diamond)$ E. corrodens; empty symbol: $12.2 \mathrm{wt} \%$, full symbol: $25.7 \mathrm{wt} \%$ metronidazole. in most cases. A one-day difference appeared in growth inhibition for P. micra at 12.2 and $25.7 \mathrm{wt} \%$ metronidazole contents. In the other cases, disks with a larger metronidazole content provided slightly larger inhibition zones than systems containing less drug on most days. Bacterial growth inhibition is shorter, only 2-3 days for A. actinomycetemcomitans, E. corrodens and $P$. micra, while the growth of $F$. nucleatum and $P$. intermedia was affected for a longer time, for 13 days.

The results of our measurements agree well with those published in the literature, suggesting that $F$. nucleatum and $P$. intermedia are more susceptible to metronidazole than A. actinomycetemcomitans, E. corrodens and P. micra, which may be completely resistant or minimally sensitive to the antimicrobial drug used. The diameter of the inhibition zone increases slightly with increasing metronidazole concentration, probably because of longer diffusion paths resulting in enhanced inhibition. The differences in the diameter of the inhibition zone are more pronounced for strains with larger susceptibility to metronidazole and they increase with time as well. Inhibition was observed in the growth of susceptible bacteria for as long as almost 2 weeks, indicating that our devices can be efficient for a long time. This fact, however, needs some consideration, since the dissolution study indicated that most of the drug is released from the disks in $24 \mathrm{hrs}$. The contradiction might be explained by the difference in the conditions, but also in the presence of metronidazole located within the polymer in the form of dissolved molecules or precipitated crystals. The diffusion, thus the release rate of metronidazole is much slower in this latter case than for the drug located among the fibers in crystal form. A slower rate leads to prolonged inhibition times, which could result in greater patient compliance and better results of the periodontitis treatment.

\section{Conclusion}

The XRD study of drug release devices prepared from electrospun PLA fibers showed that the polymer was partially crystalline in both devices, i.e., in fiber mats and disks. A part of metronidazole precipitated and was located in the form of crystals among the fibers. The penetration of the aqueous medium and dissolution were different in the two devices, they were faster in disks and slower in fiber mats, due to the morphology of the device and because of the action of capillary forces. Disks released the drug much faster than fiber mats. The microbiological study carried out with five bacterial strains confirmed results published in the 
open literature that some strains are insensitive to metronidazole (A. actinomycetemcomitans, E. corrodens, $P$. micra), while the drug is very efficient against others ( $F$. nucleatum, $P$. intermedia). Although the release study indicated the fast dissolution of the drug, the concentration achieved a plateau value in $24 \mathrm{hrs}$ for the disks; the inhibition effect was much longer, 13 days for bacteria sensitive to metronidazole. The longer inhibition period could be explained by the slower diffusion of metronidazole located inside the fibers of the devices and the slow penetration of the aqueous medium into them. The results indicated that in all probability the devices prepared may be effective in the treatment of periodontitis.

\section{Acknowledgments}

Zita Zuba is acknowledged for carrying out preliminary experiments and optimization of fiber spinning for the devices tested. We are grateful to Judit Rebeka Molnar for the preparation of the fibers used in the experiments and to Péter Polyák for the fitting of dissolution results. The National Research, Development and Innovation Office (NKFIH, Grant No. K 120039) is acknowledged for the financial support of the research. The University of Szeged Open Access Fund (FundRef, Grant No. 4383) is also acknowledged for funding the open-access publication. This research was supported by the ÚNKP-193-SZTE-175 New National Excellence Program of the Ministry for Innovation and Technology.

\section{Disclosure}

The authors report no conflicts of interest in this work.

\section{References}

1. Bosshardt DD. The periodontal pocket: pathogenesis, histopathology and consequences. Periodontol 2000. 2018;76(1):43-50. doi:10.1111/ prd. 12153

2. Lamont RJ, Koo H, Hajishengallis G. The oral microbiota: dynamic communities and host interactions. Nat Rev Microbiol. 2018;16 (12):745-759. doi:10.1038/s41579-018-0089-x

3. Sheiham A, Netuveli GS. Periodontal diseases in Europe. Periodontol 2000. 2002;29(1):104-121. doi:10.1034/j.1600-0757.2002.290106.x

4. Eick S, Nydegger J, Bürgin W, Salvi GE, Sculean A, Ramseier C. Microbiological analysis and the outcomes of periodontal treatment with or without adjunctive systemic antibiotics - a retrospective study. Clin Oral Investig. 2018;22(9):3031-3041. doi:10.1007/s00784-0182392-3

5. Jain N, Jain GK, Javed S, et al. Recent approaches for the treatment of periodontitis. Drug Discov Today. 2008;13(21-22):932-943. doi:10.1016/ j.drudis.2008.07.010

6. Schwach-Abdellaoui K. Local delivery of antimicrobial agents for the treatment of periodontal diseases. Eur J Pharm Biopharm. 2000;50 (1):83-99. doi:10.1016/S0939-6411(00)00086-2
7. Southard GL, Godowski KC. Subgingival controlled release of antimicrobial agents in the treatment of periodontal disease. Int $J$ Antimicrob Agents. 1998;9(4):239-253. doi:10.1016/S0924-8579(98)00004-1

8. Yar M, Farooq A, Shahzadi L, et al. Novel meloxicam releasing electrospun polymer/ceramic reinforced biodegradable membranes for periodontal regeneration applications. Mater Sci Eng C. 2016;64:148-156. doi:10.1016/j.msec.2016.03.072

9. Do MP, Neut C, Metz H, et al. Mechanistic analysis of PLGA/ HPMC-based in-situ forming implants for periodontitis treatment. Eur J Pharm Biopharm. 2015;94:273-283. doi:10.1016/j.ejpb. 2015.05.018

10. Tyagi P, Vaish S, Dodwad V. Clinical efficacy of subgingivally delivered $0.5 \%$ controlled release azithromycin gel in the management of chronic periodontitis. Indian J Med Sci. 2011;65(6):223-230. doi:10.4103/00195359.107017

11. Rams TE, Slots J. Local delivery of antimicrobial agents in the periodontal pocket. Periodontol 2000. 1996;10(1):139-159. doi:10.1111/j.1600-0757.1996.tb00072.x

12. Liu M, Duan X-P, Li Y-M, Yang D-P, Long Y-Z. Electrospun nanofibers for wound healing. Mater Sci Eng C. 2017;76:1413-1423. doi:10.1016/j.msec.2017.03.034

13. Zahedi P, Rezaeian I, Ranaei-Siadat SO, Jafari SH, Supaphol P. A review on wound dressings with an emphasis on electrospun nanofibrous polymeric bandages. Polym Adv Technol. 2010;21 (2):77-95. doi:10.1002/pat.1625

14. Thakkar S, Misra M. Electrospun polymeric nanofibers: new horizons in drug delivery. Eur J Pharm Sci. 2017;107(May):148-167. doi:10.1016/j.ejps.2017.07.001

15. Sill TJ, von Recum HA. Electrospinning: applications in drug delivery and tissue engineering. Biomaterials. 2008;29(13):1989-2006. doi:10.1016/j.biomaterials.2008.01.011

16. Zamani M, Prabhakaran MP, Ramakrishna S. Advances in drug delivery via electrospun and electrosprayed nanomaterials. Int $J$ Nanomedicine. 2013;8:2997-3017. doi:10.2147/IJN.S43575

17. Asmatulu R, Khan WS. Introduction to electrospun nanofibers. Synth Appl Electrospun Nanofibers. 2019;1-15. doi:10.1016/B978-0-12813914-1.00001-8

18. Chou SF, Carson D, Woodrow KA. Current strategies for sustaining drug release from electrospun nanofibers. $J$ Control Release. 2015;220:584-591. doi:10.1016/j.jconrel.2015.09.008

19. Chou SF, Woodrow KA. Relationships between mechanical properties and drug release from electrospun fibers of PCL and PLGA blends. J Mech Behav Biomed Mater. 2017;65(July2016):724-733. doi:10.1016/j.jmbbm.2016.09.004

20. Reise M, Wyrwa R, Müller U, et al. Release of metronidazole from electrospun poly(l-lactide-co-d/l-lactide) fibers for local periodontitis treatment. Dent Mater. 2012;28(2):179-188. doi:10.1016/j.dental.2011. 12.006

21. Schkarpetkin D, Reise M, Wyrwa R, et al. Development of novel electrospun dual-drug fiber mats loaded with a combination of ampicillin and metronidazole. Dent Mater. 2016;32(8):951-960. doi:10.1016/j.dental.2016.05.002

22. Monteiro APF, Rocha CMSL, Oliveira MF, et al. Nanofibers containing tetracycline/ $\beta$-cyclodextrin: physico-chemical characterization and antimicrobial evaluation. Carbohydr Polym. 2017;156:417-426. doi:10.1016/j.carbpol.2016.09.059

23. Khan G, Yadav SK, Patel RR, Kumar N, Bansal M, Mishra B. Tinidazole functionalized homogeneous electrospun chitosan/poly ( $\varepsilon$-caprolactone) hybrid nanofiber membrane: development, optimization and its clinical implications. Int J Biol Macromol. 2017;103:1311-1326. doi:10.1016/j. ijbiomac.2017.05.161

24. Kopytynska-Kasperczyk A, Dobrzynski P, Pastusiak M, Jarzabek B, Prochwicz W. Local delivery system of doxycycline hyclate based on -caprolactone copolymers for periodontitis treatment. Int J Pharm. 2015;491(1-2):335-344. doi:10.1016/j. ijpharm.2015.06.034 
25. Ranjbar-Mohammadi M, Zamani M, Prabhakaran MP, Bahrami SH, Ramakrishna S. Electrospinning of PLGA/gum tragacanth nanofibers containing tetracycline hydrochloride for periodontal regeneration. Mater Sci Eng C. 2016;58:521-531. doi:10.1016/j.msec.2015.08.066

26. Amani H, Arzaghi H, Bayandori M, et al. Controlling cell behavior through the design of biomaterial surfaces: a focus on surface modification techniques. Adv Mater Interfaces. 2019;6(13):1900572. doi:10.1002/admi.201900572

27. Li ZQ, Zhou XD, Pei CH. Synthesis of PLA-co-PGMA copolymer and its application in the surface modification of bacterial cellulose. Int J Polym Mater. 2010;59(9):725-737. doi:10.1080/00914037.2010.483214

28. Jordá-Vilaplana A, Fombuena V, García-García D, Samper MD, Sánchez-Nácher L. Surface modification of polylactic acid (PLA) by air atmospheric plasma treatment. Eur Polym J. 2014;58:23-33. doi:10.1016/j.eurpolymj.2014.06.002

29. Biresaw G, Carriere CJ. Interfacial tension of poly(lactic acid)/polystyrene blends. J Polym Sci B Polym Phys. 2002;40(19):2248-2258. doi:10.1002/polb.10290

30. Kenyó C, Kajtár DA, Renner K, Kröhnke C, Pukánszky B. Functional packaging materials: factors affecting the capacity and rate of water adsorption in desiccant composites. J Polym Res. 2013;20(11):294. doi:10.1007/s10965-013-0294-2

31. Zhang JJ, Liu J, Yu H, Zhang Y, Zhu MF, Chen YM. Crosslinked electrospun UPM/PHBV/PVP fibers for sustained drug release. Mater Sci Forum. 2009;610-613:1331-1334. doi:10.4028/www.scientific. net/MSF.610-613.1331

32. Yohe ST, Colson YL, Grinstaff MW. Superhydrophobic materials for tunable drug release: using displacement of air to control delivery rates. J Am Chem Soc. 2012;134(4):2016-2019. doi:10.1021/ja211148a

33. Moore WEC, Moore LVH. The bacteria of periodontal diseases. Periodontol 2000. 1994;5(1):66-77. doi:10.1111/j.1600-0757.1994. tb00019.x

34. Costalonga M, Herzberg MC. The oral microbiome and the immunobiology of periodontal disease and caries. Immunol Lett. 2014;162 (2):22-38. doi:10.1016/j.imlet.2014.08.017

35. Darby I, Curtis M. Microbiology of periodontal disease in children and young adults. Periodontol 2000. 2001;26:33-53. doi:10.1034/ j.1600-0757.2001.2260103.x

36. Tsai C-Y, Tang CY, Tan T-S, Chen K-H, Liao K-H, Liou M-L. Subgingival microbiota in individuals with severe chronic periodontitis. J Microbiol Immunol Infect. 2016;1-9. doi:10.1016/j. jmii.2016.04.007

37. Wang J, Qi J, Zhao $\mathrm{H}$, et al. Metagenomic sequencing reveals microbiota and its functional potential associated with periodontal disease. Sci Rep. 2013;3(1):1843. doi:10.1038/srep01843

38. Laksmana T, Kittichotirat W, Huang Y. Metagenomic analysis of subgingival microbiota following non-surgical periodontal therapy: a pilot study. Open Dent J. 2012;6(1):255-261. doi:10.2174/ 1874210601206010255
39. Chen H, Liu Y, Zhang $M$, et al. A filifactor alocis-centered co-occurrence group associates with periodontitis across different oral habitats. Sci Rep. 2015;5(1):9053. doi:10.1038/srep09053

40. Liu B, Faller LL, Klitgord N, et al. Deep sequencing of the oral microbiome reveals signatures of periodontal disease. Highlander SK, ed. PLoS One. 2012;7(6):e37919. doi:10.1371/journal.pone.0037919

41. Könönen E, Müller H-P. Microbiology of aggressive periodontitis. Periodontol 2000. 2014;65(1):46-78. doi:10.1111/prd.12016

42. Kinane DF. Causation and pathogenesis of periodontal disease. Periodontol 2000. 2001;25(1):8-20. doi:10.1034/j.1600-0757.2001. 22250102.x

43. Socransky SS, Haffajee AD. Dental biofilms: difficult therapeutic targets. Periodontol 2000. 2002;28(1):12-55. doi:10.1034/j.16000757.2002.280102.x

44. Sanz M, Beighton D, Curtis MA, et al. Role of microbial biofilms in the maintenance of oral health and in the development of dental caries and periodontal diseases. Consensus report of group 1 of the joint EFP/ORCA workshop on the boundaries between caries and periodontal disease. J Clin Periodontol. 2017;44:S5-S11. doi:10.1111/jepe.12682

45. Chen CKC, Wilson ME. Eikenella corrodens in human oral and non-oral infections: a review. J Periodontol. 1992;63(12):941-953. doi:10.1902/jop.1992.63.12.941

46. Baghban A, Gupta S. Parvimonas micra: a rare cause of native joint septic arthritis. Anaerobe. 2016;39:26-27. doi:10.1016/j.anaerobe.2016.02.004

47. Rams TE, Feik D, Listgarten MA, Slots J. Peptostreptococcus micros in human periodontitis. Oral Microbiol Immunol. 1992;7(1):1-6. doi:10.1111/j.1399-302X.1992.tb00011.x

48. Santos FA, Bastos EMA, Rodrigues PH, et al. Susceptibility of prevotella intermedia/prevotella nigrescens (and porphyromonas gingivalis) to propolis (bee glue) and other antimicrobial agents. Anaerobe. 2002;8(1):9-15. doi:10.1006/anae.2002.0411

49. Han YW. Fusobacterium nucleatum: a commensal-turned pathogen. Curr Opin Microbiol. 2015;23(3):141-147. doi:10.1016/j.mib.2014.11.013

50. Jacinto RC, Montagner F, Signoretti FGC, Almeida GC, Gomes BPFA. Frequency, microbial interactions, and antimicrobial susceptibility of fusobacterium nucleatum and fusobacterium necrophorum isolated from primary endodontic infections. $J$ Endod. 2008;34(12):1451-1456. doi:10.1016/j.joen.2008.08.036

51. Raja M. Aggregatibacter actinomycetemcomitans - a tooth killer? J Clin Diagn Res. 2014;8(8):13-16. doi:10.7860/JCDR/2014/9845.4766

52. Oettinger-Barak O, Dashper SG, Catmull DV, et al. Antibiotic susceptibility of aggregatibacter actinomycetemcomitans JP2 in a biofilm. J Oral Microbiol. 2013;5(1):20320. doi:10.3402/jom.v5i0.20320

\section{Publish your work in this journal}

Drug Design, Development and Therapy is an international, peerreviewed open-access journal that spans the spectrum of drug design and development through to clinical applications. Clinical outcomes, patient safety, and programs for the development and effective, safe, and sustained use of medicines are a feature of the journal, which has also been accepted for indexing on PubMed Central. The manuscript management system is completely online and includes a very quick and fair peer-review system, which is all easy to use. Visit http://www. dovepress.com/testimonials.php to read real quotes from published authors. 